$4-1-2020$

\title{
Evidence review and analysis of provider behavior change opportunities
}

Emily Zimmerman

Vivien Caetano

Rachel Banay

Jana Smith

Follow this and additional works at: https://knowledgecommons.popcouncil.org/departments_sbsr-rh

Part of the Health Communication Commons, and the Public Health Commons How does access to this work benefit you? Let us know!

\section{Recommended Citation}

Zimmerman, Emily, Vivien Caetano, Rachel Banay, and Jana Smith. 2020. "Evidence review and analysis of provider behavior change opportunities," Breakthrough RESEARCH Final Report. Washington, DC:

Population Council. 


\section{LITERATURE REVIEW}

\section{Evidence Review and Analysis of Provider Behavior Change Opportunities}

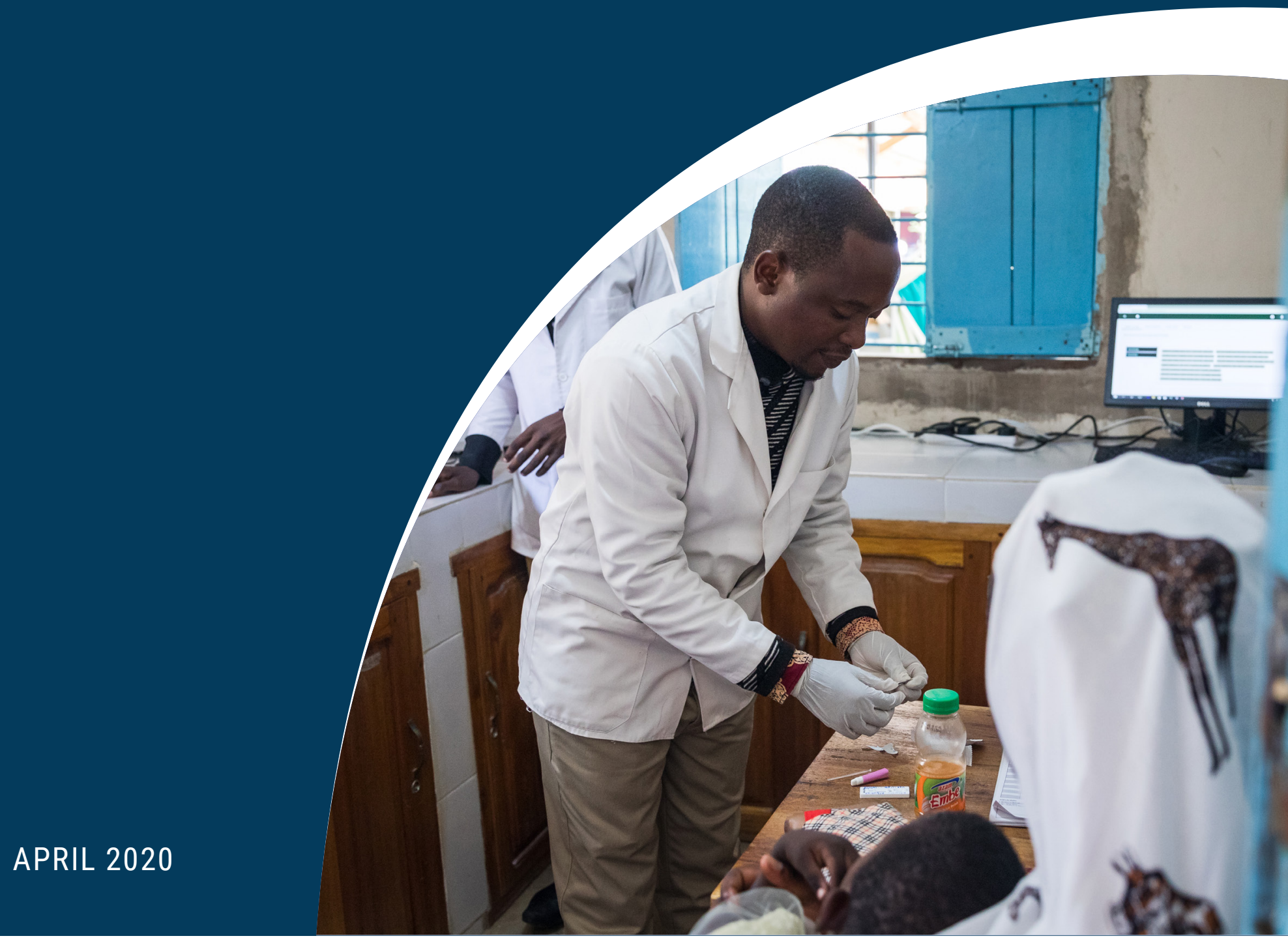




\section{USAID 0040}

Breakthrough RESEARCH is made possible by the generous support of the American people through the United States Agency for International Development (USAID) under the terms of cooperative agreement no. AID-OAA-A-17-00018. The contents of this document are the sole responsibility of the Breakthrough RESEARCH and Population Council and do not necessarily reflect the views of USAID or the United States Government.

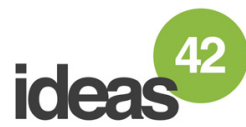

ideas42 is a non-profit that uses insights from human behavior-why people do what they do-to help improve lives, build better systems, and drive social change. For more than a decade, we've been at the forefront of applying behavioral science in the real world. Our efforts have so far extended to 40 countries as we've partnered with governments, foundations, NGOs, private enterprises, and a wide array of public institutions--in short, anyone who wants to make a positive difference in peoples' lives.

\section{- POPULATION \\ COUNCIL \\ Ideas. Evidence. Impact.}

The Population Council confronts critical health and development issuesfrom stopping the spread of HIV to improving reproductive health and ensuring that young people lead full and productive lives. Through biomedical, social science and public health research in about 50 countries, the Council works with our partners to deliver solutions that lead to more effective policies, programs, and technologies to improve lives worldwide. Established in 1952 and headquartered in New York, the Council is a nongovernmental, nonprofit organization with an international board of trustees.

\section{Breakthrough RESEARCH FOR SOCIAL \& BEHAVIOR CHANGE}

Breakthrough RESEARCH catalyzes social and behavior change (SBC) by conducting state-of-the-art research and evaluation and promoting evidence-based solutions to improve health and development programs around the world. Breakthrough RESEARCH is a consortium led by the Population Council in partnership with Avenir Health, ideas42, Institute for Reproductive Health at Georgetown University, Population Reference Bureau, and Tulane University.
(C)2020 The Population Council. All rights reserved.

Cover photo by USAID

\section{Suggested Citation}

Zimmerman, Emily, Vivien Caetano, Rachel Banay, and Jana Smith. 2020. "Evidence review and analysis of provider behavior change opportunities." Breakthrough RESEARCH Final Report. Washington DC: Population Council.

\section{Contact}

4301 Connecticut Avenue NW, Suite 280 | Washington, DC 20008 +12022379400 | BreakthroughResearch@popcouncil.org breakthroughactionandresearch.org 
Evidence Review and Analysis of Provider Behavior Change Opportunities
Emily Zimmerman
Vivien Caetano
Rachel Banay
Jana Smith

ideas 42 


\section{List of Acronyms}

$\begin{array}{ll}\text { ACT } & \text { Artemisinin-based Combination Therapy } \\ \text { ART } & \text { Antiretroviral Therapy } \\ \text { ANC } & \text { Antenatal Care } \\ \text { DOT } & \text { Directly Observed Therapy } \\ \text { CHW } & \text { Community Health Worker } \\ \text { COPE } & \text { Client-oriented, Provider-efficient } \\ \text { FPRH } & \text { Family Planning and Reproductive Health } \\ \text { HIV } & \text { Human Immunodeficiency Virus } \\ \text { iCCM } & \text { Integrated Community Case Management } \\ \text { IMCI } & \text { Integrated Management of Childhood Illnesses } \\ \text { IPTp } & \text { Intermittent Preventive Treatment of Malaria in Pregnancy } \\ \text { ITN } & \text { Insecticide-treated Bed Net } \\ \text { LARC } & \text { Long-acting Reversible Contraception } \\ \text { MNCH } & \text { Maternal, Newborn, and Child Health } \\ \text { MOI } & \text { Missed Opportunities for Immunization } \\ \text { PITC } & \text { Provider-initiated HIV Testing and Counseling } \\ \text { POC } & \text { Point-of-care } \\ \text { PMTCT } & \text { Prevention of Mother-to-child Transmission } \\ \text { PNC } & \text { Postnatal Care } \\ \text { RCT } & \text { Randomized Controlled Trial } \\ \text { RDT } & \text { Rapid Diagnostic Test } \\ \text { SBC } & \text { Social and Behavior Change } \\ \text { SCC } & \text { Safe Childbirth Checklist } \\ \text { SDG } & \text { Sustainable Development Goal } \\ \text { SRH } & \text { Sexual and Reproductive Health } \\ \text { STI } & \text { Sexually Transmitted Infection } \\ \text { UNICEF } & \text { The United Nations Children's Fund } \\ \text { VCAT } & \text { Values Clarification and Attitude Transformation } \\ \text { WHO } & \text { World Health Organization } \\ & \end{array}$




\section{Table of Contents}

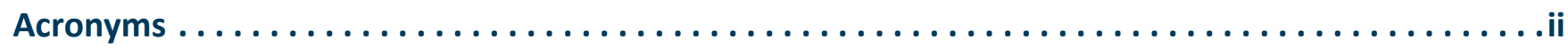

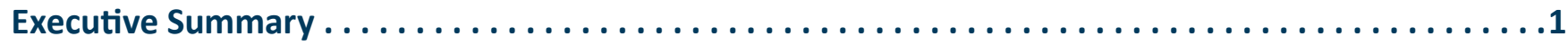

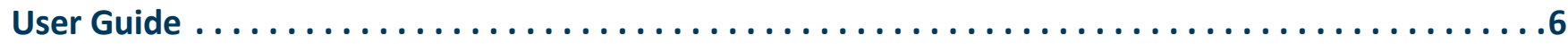

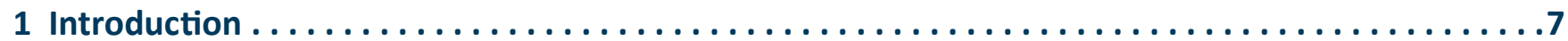

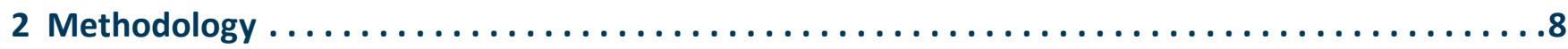

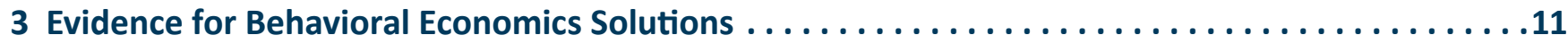

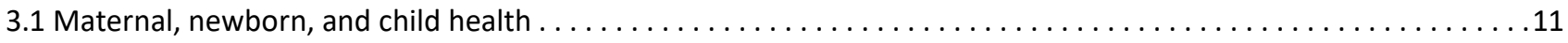

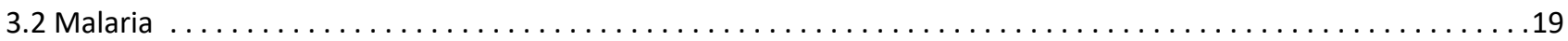

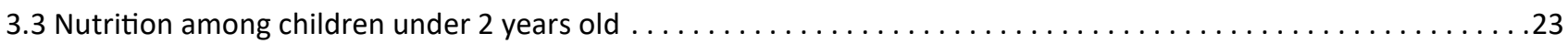

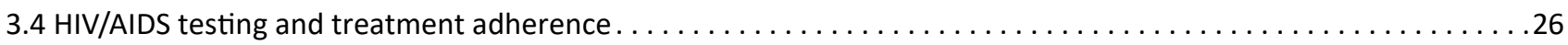

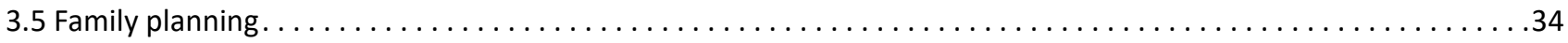

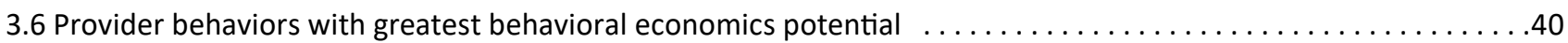

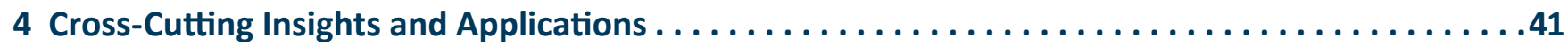

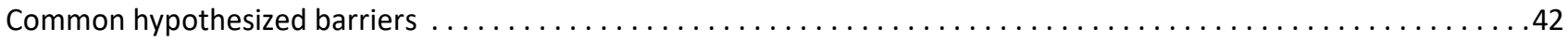

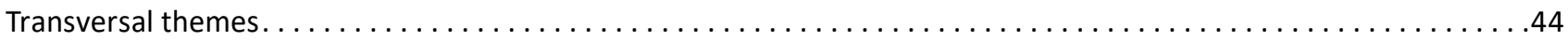

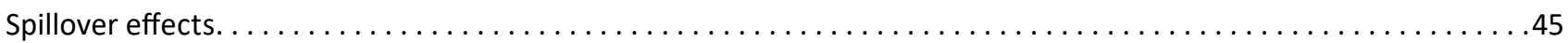

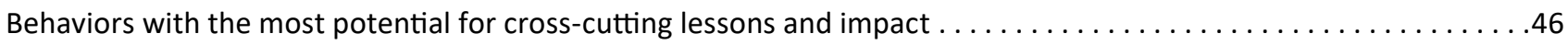

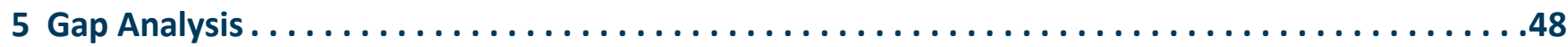

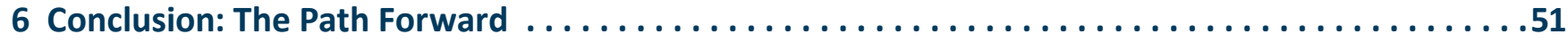

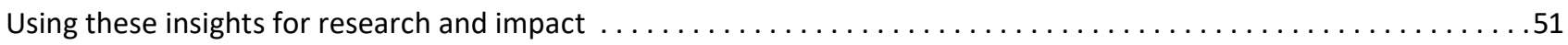

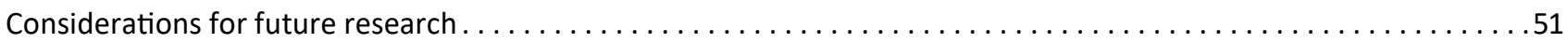

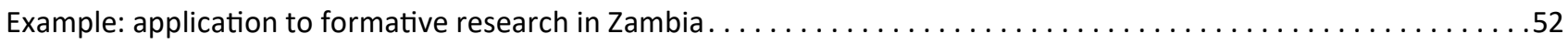

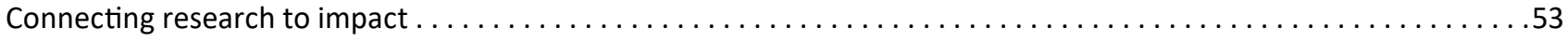

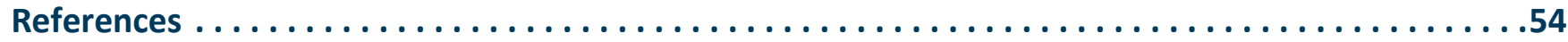


IV EVIDENCE REVIEW AND ANALYSIS OF PBC OPPORTUNITIES 


\section{Executive Summary}

The field of global health is increasingly starting to recognize the potential for interventions informed by behavioral economics to address pressing health needs. ${ }^{1-3}$ Research from behavioral economics and other behavioral sciences has increasingly been used to strengthen programming by providing a novel lens for understanding behavior. This approach entails identifying contextual features which affect how individuals make relevant decisions and take action, and then designing solutions that can change this context or mitigate the impact of contextual barriers to improve health within existing practical constraints.

However, only limited research and programmatic work to date has focused on health care providers' behaviors and interventions to support them. Just as patients may act in ways that do not always appear to align with their health interests or intentions, providers may also act sub-optimally in ways that affect patient health. The complexity of providing health care services in low-resource settings amplifies many of these behavioral challenges, and the stakes in these settings are particularly high. Providers' communication and interaction with patients influences service uptake and utilization; their adherence to quality standards determines the clinical effectiveness of the services they provide; and through a single interaction they can exert profound influence on a patient's future care-seeking and self-care behaviors.

This paper reviews the evidence on the promise of behavioral economics to improve health outcomes through provider-facing interventions in five critical health areas. Our analysis draws from the limited existing evidence base on this topic to suggest where and how behavioral economics interventions may be most impactful and where further research may contribute most to building the knowledge base.

\section{Methodology}

This review is not intended to be a systematic or comprehensive review of the evidence on provider behaviors, but to gather evidence from a wide range of sources on the potential for provider behavior change interventions. Since there is not a clear definition of high-impact provider behaviors in the literature, we first sought to define prospective provider behaviors. Because this review is intended to inform further field research in Zambia, we focused on 27 provider behaviors in five health areas that are particularly critical in the Zambian context: family planning, maternal, newborn, and child health, child nutrition, malaria, and HIV/AIDS.
The promise of behavioral economics: review of evidence

For each of the 27 provider behaviors, we reviewed the evidence on three questions:

1. What is the potential link between this provider behavior and health outcomes?

2. What interventions (behavioral and otherwise) have attempted to improve this provider behavior, and what is the evidence of their effectiveness?

3. To what extent are the challenges driven by behavioral, rather than structural (i.e., infrastructure or clinical skills), factors?

The following are our conclusions from this review for each prospective provider behavior: 


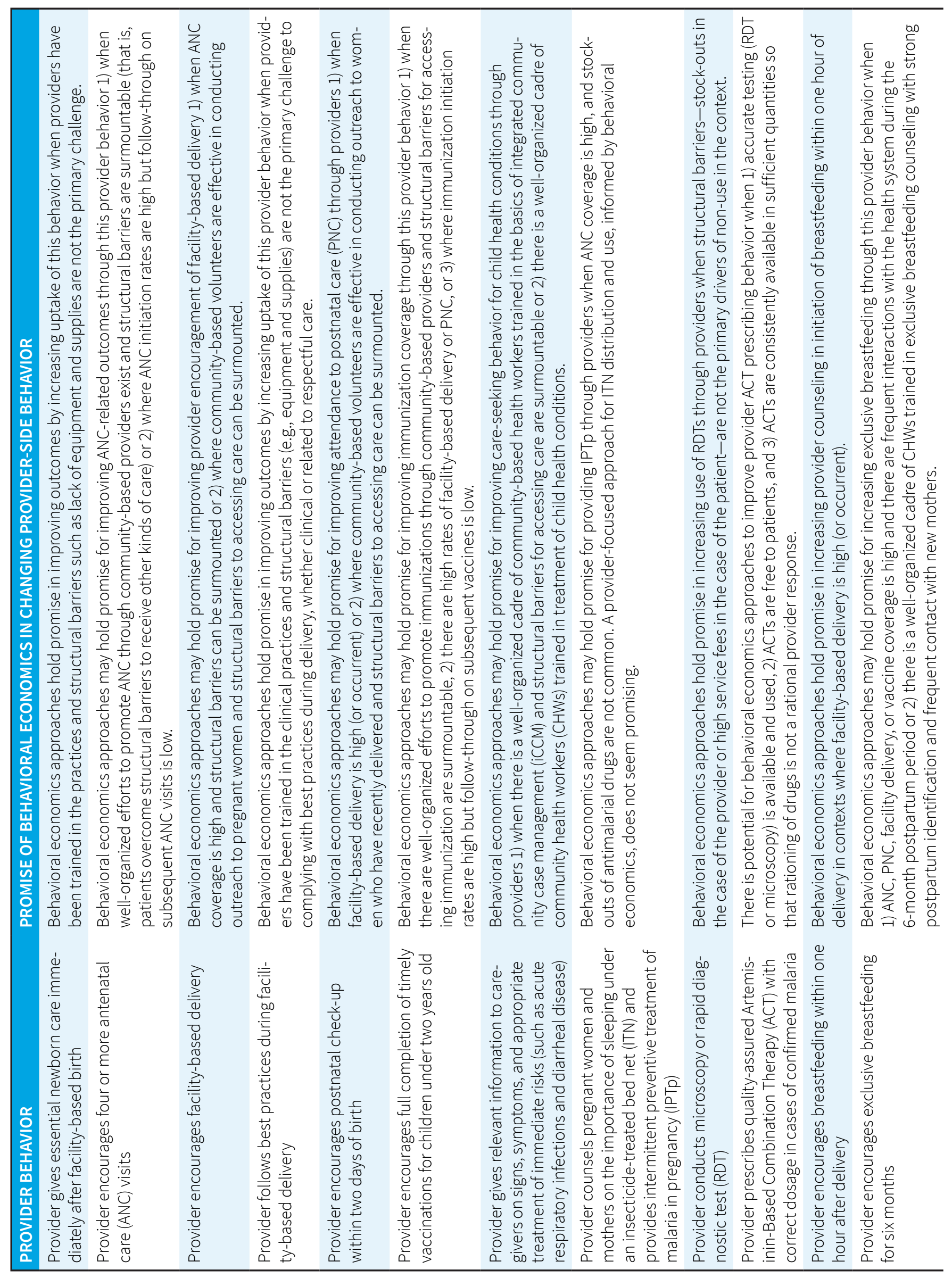




\begin{tabular}{|c|c|c|c|c|c|c|c|c|c|c|c|c|c|c|}
\hline 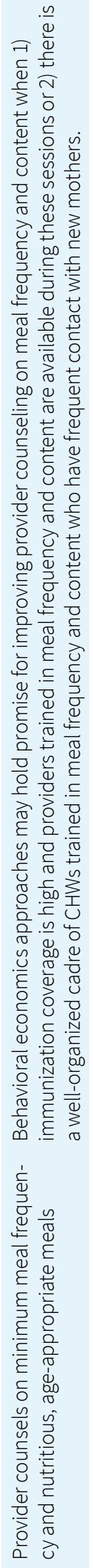 & 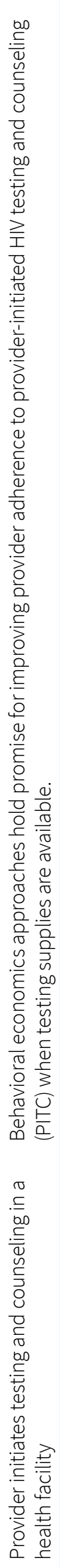 & 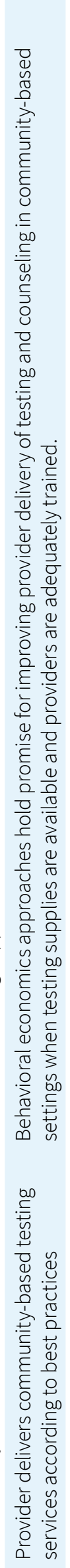 & 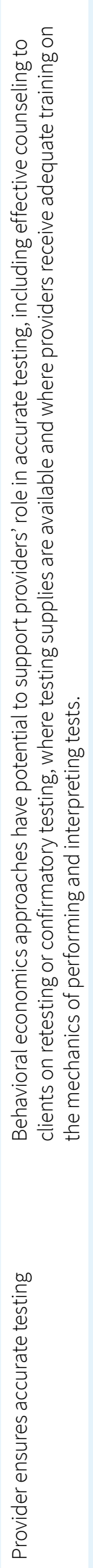 & 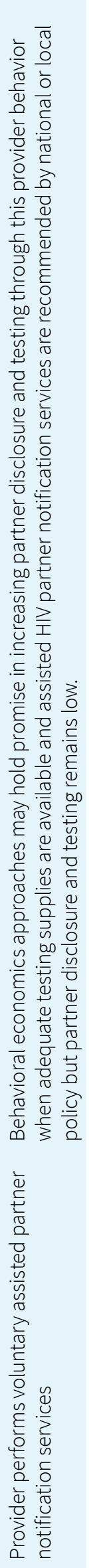 & 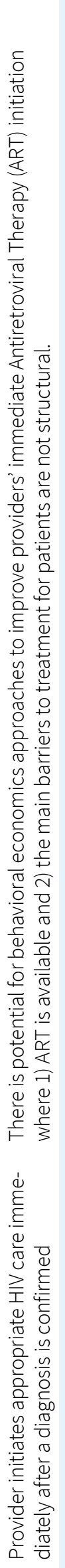 & 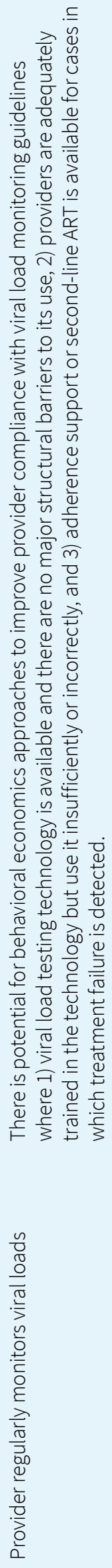 & 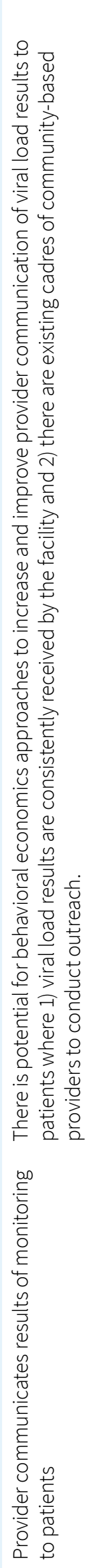 & 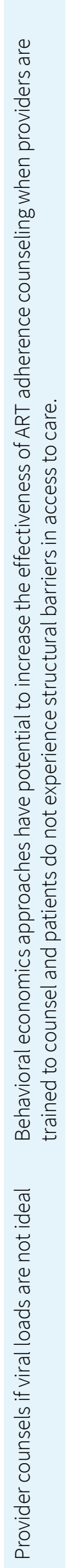 & 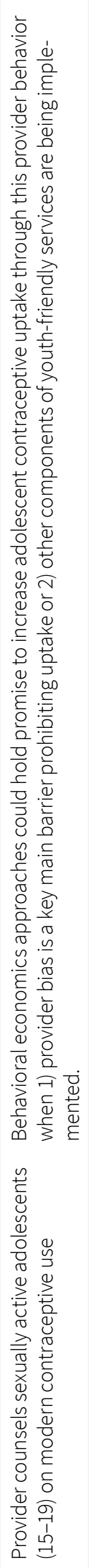 & 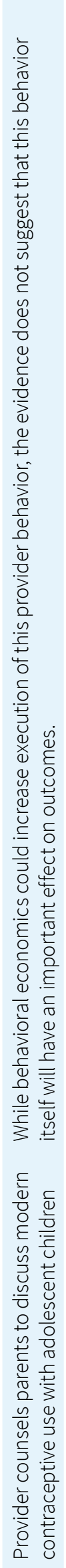 & 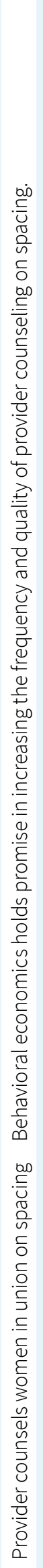 & 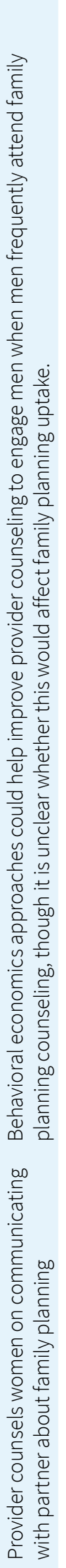 & 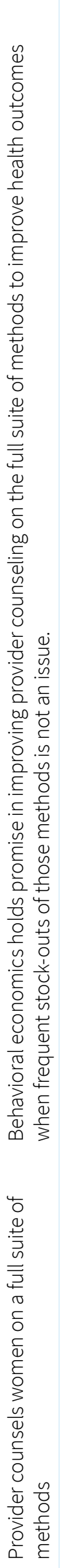 & 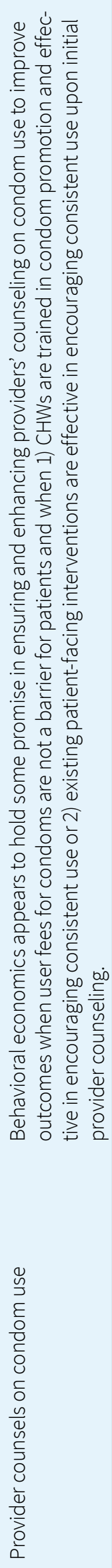 \\
\hline
\end{tabular}




\section{Cross-cutting insights and applications}

While all individual health behaviors have unique dimensions that merit independent assessment, many behaviors in the health field are interlinked. These linkages provide opportunities for impacting multiple behaviors or identifying places where lessons can be leveraged to influence programming on different behaviors. Cross-cutting insights and applications arise from three different angles:

- Behavioral barriers arise from features of the context in which providers live and work that trigger universal psychological tendencies, and similar barriers may appear across quite different provider behaviors. Understanding how cues in one context contribute to a behavioral barrier for a single provider behavior can generate insights into how a similar barrier might arise elsewhere for a different provider or behavior.

- Transversal themes are the common manifestations of various barriers in the way providers act, such as their response to a new technology or guideline. An understanding of when and how transversal themes result from behavioral barriers opens the door to applying components of one solution to another health issue or behavior.

- Spillover effects consider ways in which the immediate effects of one behavior may increase the likelihood of future interactions with the health system in a way that could affect other health outcomes by directly or indirectly surmounting patient-side barriers. Understanding the spillover effects of provider behaviors allows us to focus our efforts on the most impactful behaviors (and interventions) and eventually to move toward more comprehensive solutions that support provider behavior more broadly.

\section{Gaps in the evidence}

Provider-facing interventions fall into several general categories. In the review, we considered interventions that are classically considered SBC interventions, interventions that leverage behavioral economics in their design, and interventions that could be further enhanced with behavioral economics approaches. While the evidence base is limited across most intervention types and for most provider behaviors, some trends emerged from our review.
- Training and education interventions reinforce or supplement providers' existing knowledge. While they are common (and frequently recommended) and some have demonstrated efficacy, the evidence of their impact on provider behavior is mixed.

- Managerial approaches involve supportive supervision, mentorship, or peer support or the implementation of audits, targets, monitoring, or feedback to improve performance. They have been implemented more frequently and have shown more success than other intervention types.

- Institutional process improvements include checklists or case sheets to enhance provider behaviors across facilities or groups, and individual process improvements target individuals' performance through microplanning devices, job aids, and targeted reminders. The limited evidence available for these types of interventions suggests that they are promising, although they have not been implemented or studied as frequently as other types of interventions.

- Supplementary patient-facing materials furnish assistance to providers primarily when they counsel, explain, or otherwise convey information to patients. Few of the programs identified in our review employed these materials.

- Financial incentives reward providers for a desired behavior. Few of the programs identified in our review linked financial incentives with the specific provider behaviors of focus, although there is a broader body of evidence on financial incentives for health care providers showing mixed results.

\section{The path forward}

Our aim is for insights from this review to be used to inform two types of critical decisions about programming and research on provider behavior change:

- Designing and adapting programs to maximize impact, adapting proven solutions to new contexts, employing lessons from the literature to address different challenges, and making strategic decisions about where and how to intervene for maximum impact on health outcomes.

- Shaping the research agenda to fill critical gaps in understanding of provider behavior, focusing research efforts on behaviors and contexts that: 
- Have strong potential for behavioral economics solutions to impact health outcomes by increasing the likelihood that a provider performs an action or improving the way in which they do so.

- Been the subject of limited existing research and/ or show early promise of impact.

- Share hypothesized barriers and transversal themes with other behaviors, suggesting potential to generate cross-cutting insights.

- Have potentially great spillover effects on other health behaviors and outcomes, suggesting that effective solutions may be particularly impactful.

- Are conducive to behavioral economics solutions, because in context there are gaps in performance of the provider behavior that are not primarily due to structural constraints.

The two aims of shaping research and maximizing impact are interconnected. As carefully scoped and designed research fills gaps in understanding of behavioral economics approaches to provider behavior change, these lessons can translate to improvements in programming. 


\section{User Guide}

\section{IF YOU'RE INTERESTED IN:}

Reviewing the evidence base for provider-

facing interventions in a specific health area

\section{TURN TO:}

Section 3, which contains brief summaries of evidence for provider-facing interventions in:

- Maternal, newborn, and child health (Section 3.1)

- Malaria (Section 3.2)

- Child nutrition (Section 3.3)

- HIV/AIDS (Section 3.4)

- Family planning (Section 3.5)

Understanding how and under what circumstances a behavioral economics approach might improve provider behavior in a particular health area

Section II, which describes the promise of behavioral economics to improve provider behaviors in:

- Maternal, newborn, and child health (Section 3.1)

- Malaria (Section 3.2)

- Child nutrition (Section 3.3)

- HIV/AIDS (Section 3.4)

- Family planning (Section 3.5)

Seeing how behavioral economics applies to provider behavior across health areas

Section 4, which provides an overview of some common "behavioral barriers" that may impede desired actions for multiple provider behaviors and health areas, as well as the common manifestations of these barriers in the way providers act.

Identifying "proven" interventions to improve provider behaviors across health areas

Section 5, which classifies the availability and strength of the evidence across health areas and intervention types and identifies provider behaviors and intervention types for which the evidence of effectiveness is strongest.

Prioritizing research in an area that has not yet been deeply explored
Section 5, which classifies the availability and strength of the evidence across health areas and intervention types and identifies provider behaviors and intervention types that show promise but for which the evidence is currently limited. 


\section{Introduction}

The field of global health is increasingly recognizing the potential for interventions informed by behavioral economics to address pressing health needs. ${ }^{1-3}$ While structural interventions such as new therapies and additional personnel are critical to functioning health systems, these approaches do not always yield expected health gains and may require resources that are not available. ${ }^{4}$ The field of social and behavior change (SBC) has been very effective in complementing these structural approaches to address underlying social norms and knowledge gaps that may hinder attainment of strong health outcomes. As the SBC field has grown and evolved, research from behavioral economics and other behavioral sciences has increasingly been used to strengthen programming by providing a novel lens for understanding behavior. This approach entails identifying contextual features that affect how individuals make relevant decisions and take action, and then designing solutions that can change this context or mitigate the impact of contextual barriers to improve health within existing practical constraints.

Surprisingly, less SBC research and programmatic work has focused on provider behaviors and interventions. Just as patients behave in ways that do not always appear to align with their health interests or intentions, providers may also act sub-optimally in ways that affect patient health. For example, providers' communication and interaction with patients influences service uptake and utilization. The extent to which providers adhere to quality standards determines the clinical effectiveness of the services they provide. Often through single interactions with patients, they profoundly affect a patient's future care-seeking and self-care behaviors.
Targeted provider behavior change interventions have the potential to lead to important health gains while leveraging existing personnel, resources, and touchpoints. Evidence suggests both reasons for shortfalls in providers' practices as well as ways these shortfalls could be addressed using behavioral economics approaches. While the field of provider behavior change in general is ripe for innovation, there are also unexplored areas of opportunity where SBC approaches, particularly behavioral economics, could be employed to address provider behaviors that have traditionally been considered exclusively as health systems strengthening work. While technical skills are requisite for behaviors in the clinical realm, once basic training has occurred the same attitudes, beliefs, and motivations that may affect bias toward patients may also influence provider adherence to clinical practices. Given the tremendous value-add that SBC approaches could offer to health systems work, provider behaviors included in this review consider areas that are more commonly believed to be in the SBC realm as well as clinical provider behaviors more commonly believed to be health systems strengthening work but whose underlying causes are behavioral and could therefore benefit from behavioral insights.

This evidence review and analysis identifies potential provider behaviors of focus for interventions, summarizes available evidence on the link between each such behavior and impacts on health, synthesizes evidence on interventions that have sought to address the behavior, and analyzes the potential effectiveness of behavioral economics approaches for the behavior to inform future directions of this work. 


\section{Methodology}

Each component of the literature review and analysis involved a different methodological approach.

In Section 3 on desirable provider health behaviors, given that high-impact provider behaviors are not defined in the literature, we developed a prospective list of potential provider behaviors for investigation. We began with the list of health areas identified by the USAID-Zambia mission as priority and identified related provider behaviors. To do this, we surveyed clinical standards of care and the broader literature, including reformulating USAID's Accelerator Behaviors with a provider focus. It should be noted that several of these behaviors would classically be defined as areas where SBC approaches could be applied, while others on the surface may seem to be clinical behaviors more relevant to health systems strengthening work. Given that behavioral economics approaches may be applied to a range of different approaches, both traditional SBC and not, these behaviors are also evaluated to understand how much of provider non-compliance is related to factors that are driven by context, attitudes, or beliefs rather than technical skills, or in other words could benefit from the application of behavioral insights. This broader approach expands the range of opportunities for SBC approaches to affect health with behavioral economics. The initial list of provider behaviors is found in Table 1.

Next, we assessed the link between health outcomes of interest in each health area and each identified provider behavior to determine whether or not increasing the behavior had the potential for positive health impact. Through this process, we ruled out behaviors in which provider actions were shown or hypothesized not to improve associated health outcomes significantly. (While we limited our review to interventions focused specifically on these selected behaviors, we recognize that research on other provider behaviors may in some cases be relevant.)

Within each health area, we then reviewed each provider behavior individually, providing an overview of its relationship with health outcomes. We then provided a summary of provider-oriented interventions that appeared to target the behavior, describing the intervention types and the results of any relevant evaluations where possible. As noted previously, given that behavioral economics approaches could be applied to channels and intervention types not considered classically as SBC, we included in our review any intervention that sought to change provider behavior, with the exception of technical trainings that were oriented around building new clinical skills. While this process was not intended to be comprehensive, we attempted to understand the universe of intervention types and draw on relevant examples as well as evidence of their efficacy. It should be noted that provider-oriented interventions were rarely evaluated as stand-alone interventions and rarely identified as "provider behavior change" in the literature and program reports. Identifying these interventions required a good degree of ingenuity, which made the process less systematic but more comprehensive. We concluded the description of each behavior with an analysis of the promise of behavioral economics to enhance it, based on hypothesized decisions and actions the provider and patient undertake and the barriers (explanations for why optimal decision-making and follow through related to the behavior is not occurring) that may be at play.

Section 4's analysis of cross-cutting insights and applications synthesized findings from Section 3 to indicate where and how provider behaviors may be interlinked, and the opportunities these linkages suggest to identify how provider-facing interventions (i.e., interventions that are focused specifically on changing the provider's behavior) could affect multiple health outcomes. The analysis did this by identifying common behavioral barriers, transversal themes, and potential spillover effects. Behavioral barriers are specific barriers that may underlie several behaviors; addressing a common barrier could thus enhance several types of behaviors. Transversal themes describe how multiple behavioral barriers might manifest into a broader area of interest in ways that could similarly affect several behaviors and health areas. Spillover effects describe how increasing uptake of a health service may increase the likelihood of future interactions with the health system in a way that promotes health by addressing patient-side barriers through provider behaviors.

The gap analysis in Section 5 drew on the provider behaviors identified in Section 3. This analysis was intended 


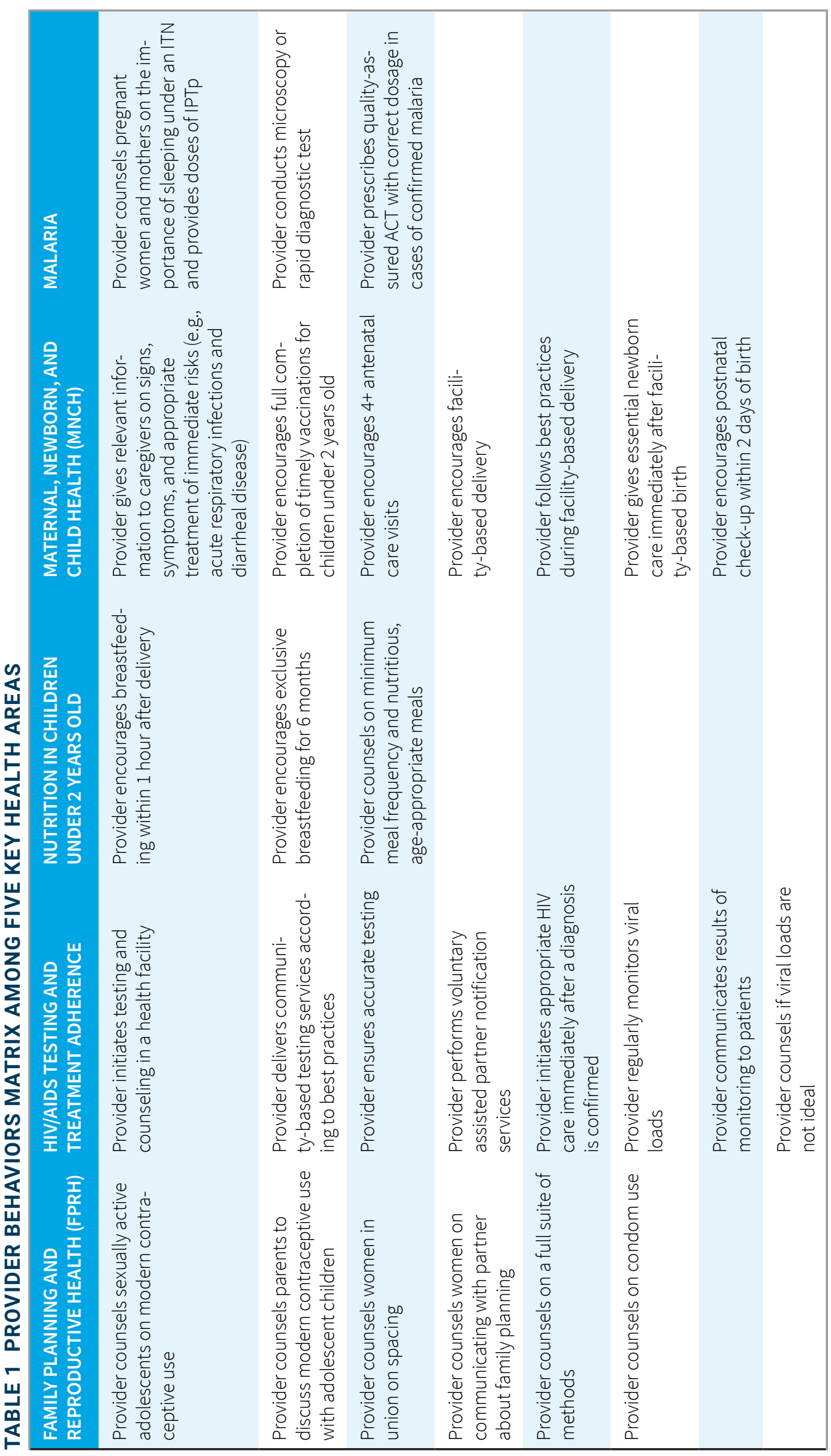


to summarize, for each desirable provider behavior described, the evidence for provider-facing interventions. We grouped interventions according to several key types that encompassed interventions commonly viewed as SBC as well as other intervention types that could leverage behavioral insights and ranked these types according to overall efficacy. For each behavior, we assessed the availability and strength of evidence for each category of intervention using a basic rubric, recognizing that our literature review was not comprehensive. Despite this fact, the exercise allowed us to portray, by provider behavior, interventions that have been tested with either promising or lackluster results, or areas without sufficient research. This analysis will inform priority areas for provider behavior change interventions and future research.

In the conclusion, we outlined how the evidence and analysis in this review could be used to design and adapting programs to maximize impact, and to shape a research agenda to fill critical gaps in understanding of provider behavior change. We then illustrated their application to choosing a provider behavior of focus for formative research in the Zambian context. 


\section{Evidence for Behavioral Economics Solutions}

This section summarizes our findings from the literature review and provides an assessment of the potential application of behavioral economics approaches to address specific provider behaviors. In the literature review, we first characterized the linkage between the specific provider behavior and relevant health outcomes to determine whether or not focusing on the provider would hold potential for more distal impact. The reason for this exercise is that specific priority provider behaviors are not well defined in the literature, nor is there clear evidence of the link between provider behaviors and outcomes. Therefore, we take this opportunity to propose and then pressure-test different provider behaviors drawing from the patient-oriented behaviors that comprise USAID's Accelerator Behaviors. Next, we identified interventions that had been developed and tested to address provider behaviors in different health areas, including approaches considered classically as SBC interventions as well as other interventions with a behavioral focus. To assess the potential application of behavioral economics approaches, we sought to rapidly envision the primary decisions and actions from both provider and patient perspectives related to the behavior and health outcomes of interest. Drawing from the literature as well as our own expertise on behavioral barriers, we hypothesized as to whether or not individual-level behavioral approaches could be effective in changing the behavior at hand, as opposed to, for instance, structural or infrastructure approaches, in a way that could conceivably impact outcomes. This assessment was not intended as a comprehensive diagnosis process of these behaviors, but instead to shed light on areas of particular promise that could be explored further with additional research.

\subsection{Maternal, newborn, and child health}

\section{Provider gives essential newborn care immedi- ately after facility-based birth}

\section{Potential link between provider behavior and health outcomes}

Most essential newborn care is applied immediately after birth with little or no client intermediation, so provider adherence to these practices has great potential to affect health outcomes of interest for facility-based births. Interventions at birth include immediate drying and providing stimulation, warmth, hygienic care, support for immediate breastfeeding, and administration of Vitamin $\mathrm{K}$, as well as a suite of other practices for babies facing particular complications. ${ }^{5}$ The World Health Organization (WHO) has articulated a set of 28 evidence-based practices to assist birth attendants in providing this care, which includes several practices related to essential newborn care specifically. ${ }^{6}$

It should be noted that structural barriers may also play a role in a provider's ability to adhere to this behavior. For instance, necessities for providing newborn care may be lacking in health facilities in low- and middle-income settings (e.g., cleaning materials, oxygen supply and delivery systems, guidelines for care). Where a safe, hygienic environment or practical equipment is unavailable, adherence to practices may be limited. ${ }^{7,8}$ Insufficient staffing or poor staffing organization have also been identified as barriers to the uptake of evidence-based practices. ${ }^{8,9}$ Nonetheless, the literature also points to the potential for behavioral approaches; one study on efforts to spread a checklist intervention found that lack of motivation, resistance to a new routine, increased workload, and the non-intuitive nature of the checklist were barriers to greater usage-all factors that are behavioral in nature. ${ }^{9}$ 


\section{Interventions focused on this behavior}

Job aids such as checklists have demonstrated an ability to improve provider practices when accompanied by appropriate training or mentoring. ${ }^{10}$ The WHO's Safe Childbirth Checklist (SCC) was recently implemented as part of a cluster randomized controlled trial (RCT) in India, along with an 8-month coaching program during which coaches (nurses) and coach leaders (physicians or public health professionals) conducted site visits to implementing facilities. These visits occurred for two to three days every two months. Coaches and coach leaders motivated facility staff to implement the Checklist and identify and resolve barriers to better patient care. At least one designated facility staff member served as a childbirth quality coordinator for each facility. While the intervention did not have a significant impact on relevant health outcomes, birth attendants' adherence to best practices increased statistically significantly in the intervention group relative to the control group.

\section{Potential application of behavioral economics approaches}

In analyzing the potential for behavioral economics approaches, we consider the dynamics of the relevant decisions and actions related to the behavior including participants, timing, and location(s). In the case of this behavior, the most relevant decisions and actions are taken by the provider with minimal patient intermediation, which makes for a relatively simple behavioral map. The time between the provider making a decision about applying these practices and the provider applying these practices (or defaulting into practices of habit) is short, which could facilitate an integrated design for addressing decision and action barriers through similar touchpoints. Furthermore, the context in which providers take actions is most likely a health facility; therefore, the relevant contextual cues (i.e., reminders or prompts) can be more easily identified and potentially modified to facilitate provider uptake of this behavior.

\section{Conclusion}

Behavioral economics approaches hold promise in increasing uptake of this provider behavior to affect outcomes when providers have been trained in the practices and structural barriers such as lack of equipment and supplies are not the primary challenge.

\section{Provider encourages four or more antenatal care visits}

\section{Potential link between provider behavior and health outcomes}

Receipt of antenatal care (ANC) can begin a positive cascade of health behaviors for women and infants. Women who receive ANC are more likely to deliver in a facility, ${ }^{11}$ and facility delivery in turn has been well documented as a means to reduce neonatal mortality. ${ }^{12}$ Some barriers to increasing ANC coverage are structural in nature, including distance, lack of transportation to the facility, and cost of services, ${ }^{13-15}$ all of which inhibit patient utilization. Among providers, lack of equipment, medicines, and supplies may partially account for low overage andquality of ANC. ${ }^{16}$ While structural barriers to providing care exist, there are ways in which behavioral approaches could enhance the early uptake and completion of recommended ANC consults.

While provider behavior would seem to be important in increasing ANC, relatively limited evidence exists on the efficacy of providers encouraging ANC as a means of driving uptake. One study showed that poor relationships or negative experiences with providers during early ANC visits deterred women from continuing to attend ANC. Fear of reprimand from providers for failing to adequately space pregnancies or for failing to attend early ANC visits also deterred women from attending ANC. ${ }^{17}$ In another study, broad quality improvement efforts in facilities led to increases in ANC initiation during the first trimester ( $8 \%$ to $24 \%$ ) and to women receiving four or more ANC visits (37\% to 64\%). ${ }^{18}$ These findings suggest that how providers deliver services may indeed impact ANC service uptake by patients. The authors did not, however, characterize the mechanisms by which these changes occurred or determine concretely which provider behavior changes were most influential in impacts to patient behavior. Therefore, it is difficult to identify the provider behaviors on which to focus to affect ANC service on the basis of this study.

\section{Interventions focused on this behavior}

A number of studies have demonstrated the potential of various forms of job aids such as pictorial booklets to increase willingness or likelihood of receiving facility-based ANC. ${ }^{19-21}$ Similar interventions have equipped community health volunteers with audio cassettes to deliver standardized messages to pregnant women and their husbands about the importance of maternal care 
and good nutrition..$^{20}$ In Nigeria, a mobile app dynamically guided community health extension workers through ANC protocols and showed a statistically significant increase in the quality of ANC administered. Completion of ANC visits by patients was not measured as an outcome, however. ${ }^{22}$

A quality improvement program in rural Kenya organized facility- and community-based providers into improvement teams to examine performance gaps in service delivery and their root causes as well as to devise and implement changes to address gaps (with coaching).

This program led to increases in the number of pregnant mothers starting ANC within the first trimester, increases in the number of mothers completing at least four ANC check-ups, and improved compliance with quality standards during ANC visits. ${ }^{18}$

In the area of ANC, performance incentives to facility-based health care providers have been applied with mixed success. One study showed that they did not increase the likelihood of more than one ANC visit or of an ANC visit in the first trimester but did lead to a significant increase in birth preparedness and in receipt of anti-tetanus vaccinations during ANC. ${ }^{23}$

\section{Potential application of behavioral economics approaches}

This behavior involves a relatively complex set of decision and action points, since both patients and providers play a critical role in ensuring four or more ANC visits. First, a patient would need to decide to attend her first ANC visit and act on this decision. This is likely to be driven more by patient-side factors than by easily identifiable provider-side factors unless community-based health workers play an active role in referring pregnant women to ANC and effectively create a moment of action or increase intention to attend ANC in the first place. Even without community mobilization, however, general perception of quality of care may play an important role in a patient's decision to initiate ANC. This perception may be derived from a range of previous interactions with the provider, including previous ANC experience or other consultations. Once the patient has attended her first ANC visit, the facility-based provider may be instrumental in ensuring follow-up with additional ANC visits by making the benefits to attending subsequent visits more salient.

\section{Conclusion}

Behavioral economics approaches focused on providers may hold promise for improving ANC-related outcomes
1) when well-organized efforts to promote ANC through community-based providers exist and structural barriers are surmountable (that is, patients overcome structural barriers to receive other kinds of care) or 2) where ANC initiation rates are high but follow-through on subsequent ANC visits is low.

\section{Provider encourages facility-based delivery}

Potential link between provider behavior and health outcomes

Similar structural barriers can be found for facility delivery as for ANC; these include distance, lack of transportation to the facility, cost of services, and lack of equipment, medicines, and supplies. ${ }^{13-16,24}$ Despite these structural barriers, evidence seems to suggest that providers can nonetheless wield influence in increasing facility-based delivery among patients. For instance, careful planning of the logistics required to arrive at a facility may help patients overcome these barriers; research suggests that providers can effectively facilitate this planning in a way that meaningfully affects outcomes. ${ }^{25,26}$

Additionally, perceived low quality of care and disrespect and abuse by providers have been noted as negativelyfecting incidence of facility-based delivery. ${ }^{27-31}$ This evidence also suggests that focusing on providers to improve the quality of respectful care could meaningfully impact a patient's decision to deliver in a facility.

\section{Interventions focused on this behavior}

Several studies have demonstrated the potential for job aids such as pictorial booklets to promote maternal health care and encourage facility delivery. ${ }^{19-21}$ Other job aids for this purpose include persuasive and testimonial videos. In one intervention, providers in India displayed these videos on their phones to serve as job aids to motivate pregnant women to deliver in facilities. While researchers felt the videos improved motivation and engagement, they also noted practical challenges familiarizing providers with the technology (formal results have not been published). ${ }^{32}$

A program implementing quality improvement efforts through facility-based teams (see Provider encourages four or more antenatal care visits, pg 12) led to an increase in facility delivery from $33 \%$ to $52 \%$. Though information on specific provider behaviors related to increasing facility-based delivery was not provided, the improvements that providers identified and implemented included coordination with traditional birth attendants, 
enhanced privacy, and streamlining of services. ${ }^{18}$ Also related to quality improvement, an intervention incorporating a 6-day training addressing major causes of maternal and newborn death and morbidity, as well as a quarterly supportive supervision system, was associated with a $30 \%$ increase in facility deliveries in Ugandan health facilities. Supervision visits were conducted by a team including a pediatrician, an obstetrician, a midwife, and a district technical officer. During visits, the team conducted skills assessments, problem solved with frontline staff, and reviewed the facility's workplan. ${ }^{33}$

Birth plans are a job aid for providers to help prepare women and their families for birth, including choosing a delivery venue, arranging transportation, and underscoring the importance of skilled delivery care in the event of potential complications. Discussing birth plans during ANC has been shown to increase facility delivery. ${ }^{26}$ Another study of birth plan preparedness cards delivered to women either by providers in facilities during ANC or by community health workers (CHWs) at home visits showed a suggestive trend toward planning for birth and complications and increases in the knowledge of danger signs. The study did not measure the impact of these changes on facility-based birth. ${ }^{25}$ In India, a phone-based dialogic message system for use by providers was tested to facilitate birth planning with patients; the intervention significantly improved quality of counseling but not health outcomes. ${ }^{34}$

Performance incentives have been applied to increase facility delivery as well. In one study in Burundi, they were associated with facility delivery among a subgroup of non-poor participants but not among the poor, suggesting the importance of addressing other patient-side barriers to increase facility-based delivery. ${ }^{35}$

\section{Potential application of behavioral economics approaches}

For this behavior, the decision and action of the patient are most relevant, but a provider could influence a patient's decision. Patients may decide whether or not they plan on delivering in a facility in advance but may change this decision in light of concerns or complications closer to the delivery date due to a variety of contextual features. Acting on this decision may require overcoming structural barriers and negotiating with partners or other family members, which providers are unlikely to be able to influence directly. However, both facility- and community-based providers may play a role in building or changing the patient's intention to deliver in a facility, for example through the counselling and services provided during ANC or through community outreach. This could be achieved, for example, by making the risks of her decision more salient or by decreasing the perceived hassles or discomfort of going to the facility. Finally, while a provider may not be able to directly help a patient overcome structural barriers to action, he or she can help ensure that a patient is not impeded from taking the final action by factors that could otherwise have been overcome by birth planning.

\section{Conclusion}

Provider-focused behavioral economics approaches may hold promise for encouraging facility-based delivery 1) when ANC coverage is high and structural barriers can be surmounted or 2) where community-based volunteers are effective in conducting outreach to pregnant women and structural barriers to accessing care can be surmounted.

\section{Provider follows best practices during facility-based delivery}

\section{Potential link between provider behavior and health outcomes}

Several best practices for facility-based delivery are applied with minimal client intermediation, so provider adherence to these practices has great potential to affect health outcomes of interest for facility-based births. The SCC articulates many evidence-based essential birth practices to guide birth attendants during delivery. ${ }^{6}$ It should be noted that providers face some structural barriers in the application of certain clinical best practices during delivery; for instance, some literature notes a lack of equipment, medicines, and supplies, which can hinder a provider's ability to comply with certain guidelines. ${ }^{7.15}$ Nonetheless, not all clinical guidelines require additional equipment, suggesting the behavioral nature of compliance of many of the practices. Furthermore, best practices during facility-based delivery include the provision of respectful maternity care, and the behavioral dimensions of respectful care are well-documented in the literature. ${ }^{27,31}$ In addition to the importance of compliance with this behavior from the perspective of clinical outcomes and human rights, quality of care during delivery and respectful care are also predictive of choice of delivery location for the next delivery ${ }^{36}$ and may affect future care-seeking behavior. 


\section{Interventions focused on this behavior}

Limited research has explored provider interventions to promote respectful care during delivery. Studies of two programs have shown that trainings, including Values Clarification and Attitude Transformation (VCAT), in respectful maternity care are feasible to implement and have the potential to reduce disrespect and abuse; they are not, however, sufficient in driving sustainable provider behavior change. ${ }^{37}$ Notably, these studies include multiple intervention elements beyond the trainings (other behavioral interventions are described below), making the impact of training alone unclear.

Provider training to increase the quality of emergency obstetric care in low-resource environments is lacking in strong evidence. ${ }^{38}$ In Mexico and Thailand, a series of workshops were used to promote providers' use of the WHO's Reproductive Health Library, an online resource on best practices in obstetric care. A randomized evaluation found that the workshops increased providers' use of the Reproductive Health Library but did not lead to sustained or consistent changes in clinical practice. ${ }^{39}$

Here again, checklists such as the SCC form part of the basis-of-care improvements, though with limited emphasis on respectful care. As described above, a program to implement the checklist in facilities in India was facilitated through coaching visits and a local program champion at the facility. The cluster RCT to evaluate this effort found that birth attendants' adherence to best practices increased statistically significantly in the intervention group relative to the control group, thus providing evidence of behavior change for this intervention. However, there was no significant difference between the treatment groups according to maternal, perinatal, or neonatal adverse outcomes. ${ }^{6}$

Audits and feedback mechanisms have been shown to lead to an absolute increase in health care professionals' compliance with desired practice. These mechanisms are effective when feedback is repetitive, provided by a supervisor or colleague, communicated in both verbal and written formats, and includes both explicit targets and an action plan. ${ }^{40}$

One such mechanism is a service charter, which has been used to generate consensus about expectations between patients and providers through a participatory process in which providers receive direct feedback from patients around respectful care. Quality improvement processes have also been used in several geographies to generate additional interventions to improve delivery of respectful care. In Tanzania, these processes led to interventions such as provider recognition with the provision of tea, best-practice sharing across wards, patient surveys to generate feedback, and adjustments to the environment such as the creation of a private admissions area and provision of curtains for privacy. As a result of this multi-component intervention, the odds of women experiencing disrespect and abuse during childbirth were reduced, though the effect of individual intervention components was not measured. ${ }^{41}$

Caring for carers is another approach that has been minimally tested to improve the provision of respectful maternal care. Counseling for providers to help them overcome experiences related to high workload, trauma, or critical incidents is one such approach. A study in Kenya found suggestive evidence that this approach, as part of a multi-component intervention that included training for providers, community-level training and dialogue, and engagement with policymakers, decreased instances of disrespectful and abusive behaviors. ${ }^{42}$

\section{Potential application of behavioral economics approaches}

In the case of this behavior, while the patient's perception is critical, the most relevant decisions and actions are taken by the provider with minimal patient intermediation. The time between the provider making a decision about applying these practices and the provider applying these practices (or defaulting into practices of habit without making an explicit decision) is short, which could facilitate an integrated design for addressing decision and action barriers. Furthermore, the context in which providers take action is most likely a health facility; therefore, the relevant contextual cues (i.e., elements in the environment that are triggering certain behaviors) can be more easily identified and modified in ways that can facilitate provider uptake of this behavior.

\section{Conclusion}

Behavioral economics approaches hold promise in increasing uptake of this provider behavior in a way that affects outcomes when providers have been trained in the clinical practices and structural barriers (e.g., equipment and supplies) are not the primary challenge to complying with best practices during delivery. 


\section{Provider encourages postnatal check-up within two days of birth}

\section{Potential link between provider behavior and health outcomes}

Postnatal care (PNC) reduces morbidity and mortality of both mothers and infants, as providers may detect and treat problems, offer support with breastfeeding, provide immunizations, and discuss birth spacing at that time. ${ }^{43}$ In more remote settings, structural as well as behavioral or socio-cultural factors may be important barriers for patients accessing PNC. After delivery, women may not feel ready to travel long distances if they live far from a facility; in addition, there may be a tradition to keep infants indoors during this initial period. ${ }^{44}$ Women may be more likely to receive PNC when they have high levels of trust in the health care system, ${ }^{45}$ which could conceivably be instilled by a respectful delivery and have been shown to be linked to receiving at least one ANC visit by a skilled provider. ${ }^{46}$ Community-based providers may be able to help patients overcome some behavioral barriers to accessing care. Nonetheless, there is relatively scant literature exploring the link between providers encouraging PNC and service uptake.

\section{Interventions focused on this behavior}

Preparedness for birth and its possible complications generally seems to have positive effects on subsequent PNC. The use of birth plans by providers during ANC has been shown to be associated with increased PNC uptake, ${ }^{26}$ as has mothers' awareness of obstetric danger signs during pregnancy ${ }^{47}$ and receiving birth preparedness messages from a Safe Motherhood Action Group during pregnancy. ${ }^{48}$

When Kenya's Ministry of Health changed PNC guidelines to improve timely coverage, a package of provider-facing interventions supported implementation. ${ }^{49}$ The interventions included a 3-day training, two supportive supervision visits, and post-partum registers in each facility. Knowledge among providers improved, with high individual-level variation (which could be attributed to the training). PNC attendance rates and timeliness improved after the policy change, but the evaluation did not consider the impact of the provider-facing intervention.

\section{Potential application of behavioral economics approaches}

In the case of PNC, while patient decisions and actions may be most critical to service uptake, there are several places along the patient journey where providers may be particularly influential and where behavioral economics approaches could be applied. For instance, the satisfaction of patients who deliver in a facility with the services they receive may positively or negatively influence their decision to return for PNC. Additionally, how the provider frames PNC during ANC or delivery (if they discuss it at all) may influence a patient's intention to attend PNC. Depending on the strength of this intention, different contextual cues may influence a patient to attend PNC or not. While many of these cues may not be related to providers, community-based providers could play an influential role in strengthening a patient's intention to attend PNC or helping to define a moment to act on this intention to spur follow-through. Community-based providers could also serve as a facilitating contextual cue in the case of women who do not deliver in a facility.

\section{Conclusion}

Provider-focused behavioral economics approaches may hold promise for improving attendance to PNC 1) when facility-based delivery is high (or occurrent) or 2) where community-based volunteers are effective in conducting outreach to women who have recently delivered and structural barriers to accessing care can be surmounted.

\section{Provider encourages full completion of timely vaccinations for children under two years old}

\section{Potential link between provider behavior and health outcomes}

Most evidence on full completion of timely vaccinations for those under two years old focuses on structural barriers rather than on elements of provider behavior. These structural barriers include factors that reduce the ability of health systems to deliver immunization services (e.g., political dynamics, infrastructure, and finances). ${ }^{50}$ Weak monitoring and information systems may also affect completion of vaccinations, ${ }^{51}$ but despite provider contributions to these shortcomings, challenges are typically framed from a systems-level supply-chain perspective.

According to WHO's 2013 Global Vaccine Action Plan, however, provider behavior has a role to play in reducing caregiver barriers. For example, lack of information and understanding about the importance of immunization among caregivers is a key barrier to initiation and 
completion of vaccines. ${ }^{52}$ Therefore, factors mentioned in the WHO Plan over which providers may exert influence include demand generation, convenient and predictably available vaccination sessions, practical counseling about why to get vaccinated and about expectations post-vaccine, and provider attitude toward patients. Providers can also reduce fear and hesitancy by addressing reports of serious adverse events following immunization. The Plan also suggests that incentivizing performance could increase vaccination among previously unreached parents, although implementation challenges must be considered and research conducted so the incentives are appropriate for their context. ${ }^{53}$

In addition to these angles, missed opportunities for immunizations (MOIs) have been noted by the WHO as a promising avenue to increase vaccination coverage. MOls are defined as "an opportunity when a person eligible for immunization and with no valid contraindication visits a health service facility and does not receive all recommended vaccines." ${ }^{154}$ In a systematic review, one of the most reported reasons for MOls among children was related to service providers. The most common specific reasons for MOls as related to service providers included not reviewing immunization cards or history, reluctance to open a vaccine vial, perceived contraindications, and not offering the immunization. ${ }^{54} \mathrm{~A}$ study in India determined that providers were less likely to verify vaccination status during illness visits as opposed to vaccination visits. ${ }^{55}$ This work suggests the importance of provider behavior in increasing full completion of vaccinations.

\section{Interventions focused on this behavior}

A strategy developed by WHO, the United Nations Children's Fund (UNICEF), and Reaching Every District includes provider-facing elements. One piece of this strategy involves health facility microplanning, which includes tools to facilitate individual health workers monitoring their own performance with targets. Interventions include operational maps accompanied by a facility session plan to ensure coverage of each site with the right delivery mechanism. Supportive supervision is another provider-facing intervention that seeks to help providers identify problems contributing to lower vaccination coverage and work to solve them; such efforts sometimes include the use of videos. ${ }^{56,57}$ Monitoring charts for visibly tracking progress on monthly or annual goals are another example of microplanning interventions aimed to affect provider behavior. These microplanning tools have been used to roll out vaccine outreach efforts-in some places more effectively ${ }^{58}$ than others ${ }^{51}$-and are believed to have been important in increasing coverage. ${ }^{59}$ While little research appears to have been conducted on the efficacy of microplanning tools, an intervention in India found that a comprehensive training among immunization staff, along with ongoing supervision by a full-time consultant, was associated with both having a monitoring chart and correctly calculating immunization dropout. ${ }^{60}$

In Guinea and Kenya, the client-oriented, provider-efficient (COPE) quality improvement program (also discussed in Section 3.3, Provider counsels on minimum meal frequency and nutritious, age-appropriate meals, page 25) led to improvements in counseling on immunization that improved mothers' knowledge and immunization coverage. ${ }^{61}$ The program consisted of self-administered assessments focused on client and staff needs, followed by tools for planning and making improvements within the facility.

Three studies evaluated the use of mobile-based apps to support health workers in increasing vaccination coverage. ${ }^{62}$ One tool created electronic vaccination records and registers but was found to have no effect on increasing vaccine coverage. Another mobile tool helped track where mobile immunization teams had been and helped decrease the number of areas that were not covered in previous campaigns. The third study measured the effectiveness of an app to support health workers in assessing families prior to a campaign and then to track uptake. It was found to help address misconceptions about vaccinations and support developing service-delivery plans. Cellular technology is also being used in other ways to drive provider behavior: Text message reminders have been used to facilitate $\mathrm{CHWs}^{\prime}$ efforts at targeting specific patients. ${ }^{62}$

In one experiment, monitoring technology coupled with individualized bonus contracts was used to improve performance in mobile polio vaccination efforts in Pakistan. ${ }^{63}$ Health workers were asked to choose a target number of vaccines that they hoped to provide during each of the two days of the campaign. They were also randomly assigned to submit this allocation either prior to the start of the campaign or the morning of the first day. Health workers with contracts tailored to their timing preferences provided significantly smoother service than those without them. Additionally, authors found that health workers were present-biased in their allocations; those who were asked to allocate the vaccines the day of the campaign were more likely to procrastinate and allocate more vaccines to the following 
day than those who were asked to allocate the vaccines in advance.

An initiative in Estonia may be one of few financial incentive schemes for provision of vaccines in a lowor middle-income country. Providers receiving these incentives met the $90 \%$ coverage rate for childhood immunization, while providers who did not receive incentives did not meet this threshold. ${ }^{64}$

Finally, while results are still pending, ideas42 has also developed interventions targeting health providers in Ethiopia to increase full immunization rates for babies. The intervention includes behaviorally informed tracking posters that display the immunization status of providers' patients, allowing them to easily survey which babies are behind schedule as well as display and take pride in their successful immunizations. In addition, providers receive text message reminders prompting them to reference the poster and perform outreach to those who drop out prior to immunization campaigns.

\section{Potential application of behavioral economics approaches}

In certain ways, this behavior has similar dynamics to that of increasing ANC attendance. First, a caregiver would need to decide to bring her child for immunization and act on this decision. There are several touchpoints where providers may be influential in shaping this initial decision. In the case of a facility-based delivery or PNC service, the provider could counsel the caregiver on the importance and timing of the first vaccine to increase her intention to follow through. Alternatively, or additionally, community-based providers who play an active role in identifying newborns for immunization may influence a caregiver's decision to get the initial immunization or spur action by helping her develop a concrete plan to attend an immunization session. Once the caregiver has brought the child for the first vaccine, the facility-based provider may be instrumental in ensuring follow-up of additional vaccines by making the benefits of additional doses salient and by clearly communicating how action can be taken. The experience of patients with the provider, such as quality of care and treatment including wait times, may also influence future patient action. Community-based providers can also play an important role in helping caregivers overcome forgetfulness by reminding them of the timing and location of subsequent vaccines close to the time when they must take action. They can also promptly refer patients to facilities should they experience side effects they attribute to the vaccine, which if unaddressed could inhibit follow-through for subsequent vaccines.

\section{Conclusion}

Behavioral economics approaches focused on providers may hold promise for improving immunization coverage 1) when there are well-organized efforts to promote immunizations through community-based providers and structural barriers for accessing immunization are surmountable, 2) where there are high rates of facility-based delivery or $\mathrm{PNC}$, or 3 ) where immunization initiation rates are high but completion rates are low.

\section{Provider gives relevant information to caregiv- ers on signs, symptoms, and appropriate treat- ment of immediate risks (such as acute respira- tory infections and diarrheal disease)}

\section{Potential link between provider behavior and health outcomes}

Provision of integrated community case management (iCCM) for common childhood illnesses by CHWs is one approach that has been widely explored in the literature to increase care-seeking among caregivers or provide treatment at a community level, thus eliminating many patient-side barriers. ${ }^{65,66}$ Much of this research, of varying degrees of quality, suggests that focusing on provider behavior in this realm, particularly on community-based providers, can effectively increase care-seeking through community mobilization and education. ${ }^{67}$ For instance, providers can work with patients to discuss folk and traditional treatments or knowledge and information barriers that may otherwise prevent clients from recognizing symptoms or understanding treatment options. ${ }^{68,69}$

Additionally, UNICEF has articulated seven different intervention models for case management of children by $\mathrm{CHWs}$, which cover options from basic management and verbal referral to integrated multiple disease case management, including treatment. ${ }^{70}$ A systematic review of these models highlights the strong evidence for impact on child mortality of pneumonia management by $\mathrm{CHWs}^{71}$ CHW-provided treatment approaches may be the most promising in settings where structural barriers such as distance from a facility or reliable transportation could otherwise affect care-seeking.

A less direct link between provider behavior and related child health outcomes concerns the perception of quality of care provided in health facilities. Given the importance of a client's previous experience at health facilities for her 
willingness to seek care from providers, helping providers offer more supportive and respectful care and de-link care from HIV-related services at upstream "gateway" touchpoints could improve the health outcome. ${ }^{68}$

\section{Interventions focused on this behavior}

One study in Rwanda tested a multi-pronged intervention focused on CHWs that showed increases in care-seeking for fever, diarrhea, and acute respiratory infections. ${ }^{65}$ The intervention included a 4-day training in iCCM, a supervisory system of monthly reports and cell coordinators, and peer support groups that leveraged peer comparison to improve performance. The authors noted peer support groups as the most promising of the programmatic components. A training program of $\mathrm{CHWs}$ combined with the provision of medical supplies also had a positive effect on care-seeking in Zambia. ${ }^{72}$ In Mali, a provider-focused iCCM training program of role-playing sessions paired with one supervisory visit was found to improve the quality of counseling to caregivers, which could potentially affect care-seeking, though this was not measured as an outcome in the study. ${ }^{73}$

Interventions have also been tested to prepare CHWs or volunteers to conduct home-based treatment of pneumonia and diarrhea, which could reduce reliance on improper treatment methods in homes while simultaneously reducing burdens on local health facilities. A training and supervision program of $\mathrm{CHWs}$ to provide treatment was tested in Bangladesh and was found to be effective and acceptable. ${ }^{74-76} \mathrm{CHW}$ s have also been shown to increase coverage of treatments such as oral rehydration therapy. For instance, Sierra Leone has particularly high coverage of oral rehydration therapy compared with other sub-Saharan African countries, a fact that has been attributed to its cadre of community volunteers, whose efficacy is similar to that of facility providers in many settings. ${ }^{74}$

\section{Potential application of behavioral economics approaches}

To ultimately drive improvements in related child health outcomes, both patients and providers must make different decisions and actions. First, a health provider must give information to the caregiver on warning signs to know when to seek care. In the case of facility-based providers, the decision to give this information and the action of doing so are likely to be closely tied in terms of timing. Behavioral economics could be used to ensure that providing this information is top of mind and that a provider does not forget to do so in the course of a consultation. The caregiver would then need to recall this information to decide to act upon it once these warning signs appear and decide to take action in seeking care. Should the information have been conveyed by the provider at a moment when the caregiver was preoccupied with other concerns or at a moment very distal from the time of symptoms, this provider-focused approach might not be as suited to support a caregiver's decision to seek care.

An alternative provider scenario for this behavior could include a community-based provider. In this case, the provider would decide whom to provide with information and could support the caregiver in the decision to seek care and act on this intent. Behavioral economics approaches could help stimulate the provider to take action, use the appropriate heuristics to identify whom to speak to, and support framing the message to most effectively stimulate action. In the case that community-based providers are also equipped with the skills to provide care, this process would be more straightforward, as the decisions and actions of the patient would be more fully in the hands of the provider.

\section{Conclusion}

Behavioral economics approaches focused on providers may hold promise for improving care-seeking behavior for child health conditions when 1) there is a well-organized cadre of community-based health workers trained in the basics of ICCM and structural barriers for accessing care are surmountable or 2) there is a well-organized cadre of community-based health workers trained in treatment of child health conditions.

\subsection{Malaria}

\section{Provider counsels pregnant women and mothers on the importance of sleeping under an insec- ticide-treated net and provides intermittent preventive treatment of malaria in pregnancy}

\section{Potential link between provider behavior and health outcomes}

Insecticide-treated nets (ITNs) and intermittent preventive treatment in pregnancy (IPTp) are recommended for effective malaria prevention in pregnant women. ${ }^{77}$ Accomplishing these activities through ANC visits is an important component of a national coverage strategy. ${ }^{78}$ There is some literature suggesting a link between 
counseling and use of ITNs. For instance, a cross-sectional study in Nigeria showed that while ITN ownership did not necessarily equate to use, women who felt confident to install an ITN and knew that it could protect pregnant women from malaria were significantly more likely to use it, indicating a potential role for provider counseling. ${ }^{79}$ Another intervention in Nigeria showed that counseling, when combined with community distribution of ITNs and IPTp, resulted in significantly greater ITN usage in treatment areas. ${ }^{80}$ Despite this link, the literature also highlights the importance of patient obstacles to ITN use, including discomfort, problems with installing ITNs, and seasonal variations in use-along with the belief that the insecticide with which nets are treated could be harmful. ${ }^{81}$ Some of these obstacles could be addressed through provider counseling, but several may not be, as implied in the literature. One evaluation in Zambia showed that CHW follow-up visits with families who received an ITNhad a modest short-term impact on use, but this impact disappeared in the medium term. ${ }^{82}$ Another study from Kenya noted an increase in ITN usage after $\mathrm{CHW}$ implementation, but usage data was self-reported and based on a pre-post comparison. ${ }^{83}$

IPTp during ANC visits has been shown to be an effective and inexpensive intervention. ${ }^{84}$ The literature notes both provider-side barriers to IPTp as well as patient barriers that could be addressed by providers. Patient barriers to IPTp include low awareness of benefits, timing, and dosage and uncertainty about the safety of taking medication during pregnancy. If IPTp is administered as intended, as directly observed therapy (DOT), few opportunities arise for the patient to defer, change her mind, or forget to take the dose. However, providers may not understand the necessity for DOT or may overestimate women's adherence when not directly observed. ${ }^{85}$ Provider barriers include poor knowledge of dosage, timing, and side effects and inaccurate estimation of gestational age, which subsequently limits the dosages a woman receives during pregnancy. Staff being too busy to prescribe or not considering the need to prescribe also hinders appropriate care, indicating that provider scarcity or automaticity may be in effect. ${ }^{86}$

\section{Interventions focused on this behavior}

In a study in India, supportive supervision combined with community mobilization was associated with statistically significant increases in ITN use, treatment-seeking, and timely diagnosis in two treatment arms relative to the control arm. Supportive supervision involved education about transmission, diagnosis, and treatment of malaria, support for performing and interpreting rapid diagnosis tests, and administration of the correct dosage of ACT and follow-up to ensure compliance. ${ }^{87}$

A study in Kenya showed that retraining of health care providers on the delivery, timing, and dosage for IPTp significantly increased coverage. ${ }^{88}$

\section{Potential application of behavioral economics approaches}

In the case of ITN use, the patient is the decision-maker and the person who must take action to affect malaria outcomes, with providers playing a lesser role. During ANC, a facility-based provider could be cued to counsel a patient on ITN use, in which case the decision and action to counsel would be closely tied. Alternatively, behavioral economics approaches could stimulate counseling by community-based providers with at-risk populations. Nonetheless, once the initial counseling has been conducted, the decision to use the ITN, and more importantly, to consistently and correctly use the ITN, is in the hands of the patient. As noted above, provider intervention, in the form of follow-up visits by $\mathrm{CHWs}$, may not be effective in guaranteeing consistent use of ITNs, thus pointing to the limitations of an approach focused on the provider. $^{82}$

In the case of providers giving doses of IPTp, a behavioral economics approach could be applied to cue providers to discuss IPTp with their patients and support providers in taking action to give treatment in cases when stockouts are not an issue. Decisions and actions are closely entwined, made in the facility context, and are in the hands of the provider, thus making it relatively straightforward to identify contextual cues which could prompt providers to give IPTp.

\section{Conclusion}

Behavioral economics approaches focused on providers may hold promise for providing doses of IPTp when ANC coverage is high and stock-outs of IPTp are not common. A provider-focused approach for ITN distribution and use informed by behavioral economics does not seem promising. 


\section{Provider conducts microscopy or rapid diagnos- tic test}

\section{Potential link between provider behavior and health outcomes}

Malaria confirmation conducted by a provider through either microscopy or a rapid diagnostic test (RDT) sets the foundation for ongoing sound malaria treatment. Importantly, a negative test result can also facilitate alternative correct diagnoses and reduce the over-prescription of antimalarials. ${ }^{89}$ Conducting the test is in the hands of the provider; therefore, the provider's behavior is tied to outcomes. A literature review on the uptake of RDTs among clinicians indicates that health workers may be acting in a climate of staff shortages and therefore affected by scarcity when failing to administer RDTs, though providers are frequently impeded by stock-outs or rationing due to low availability, suggesting the role of both behavioral and structural barriers for this behavior. ${ }^{90}$

\section{Interventions focused on this behavior}

A study in Myanmar of informal private health care providers (e.g., general retail stores, drug vendors and pharmacists), a common first point of care for febrile illness, investigated the effects of bi-monthly intensive support visits by health officers to providers, including discussions with providers and additional education, on use of RDTs. The study found that these provider-facing interventions combined with price subsidies on RDTs, were more effective in increasing use of RDTs than either price subsidies alone or price subsidies combined with financial incentives for providers. ${ }^{91}$

An intervention in Zambia among CHWs not previously trained to administer RDTs found that a job aid in the form of a detailed instructional flowchart with visuals statistically significantly increased correct administration of the RDT relative to package instructions alone. ${ }^{92}$ Use of the job aid plus a 3-hour training increased test administration even further. In an intervention in Uganda targeting registered drug-shop vendors due to their primacy treating those with febrile illnesses, training and supervision gave these providers both the technical knowledge and legitimacy they did not previously have to use RDTs, though the study did not evaluate their administration of RDTs. ${ }^{93}$ Training combined with an audit-feedback system on diagnostic performance led to improvements in conducting and properly recording diagnostic tests for malaria in Laos. ${ }^{94}$
In Tanzania, a trial tested a) interactive workshops on malaria testing and prescription together with feedback summaries on RDT use and treatments prescribed, and best practices and motivational messages delivered through SMS and b) all of those elements in addition to posters and patient-facing leaflets with Ministry of Health branding, and found that these did not result in differences in RDT testing between treatment and control groups, thus suggesting the complexity of factors that may be at play in different contexts. ${ }^{95}$

\section{Potential application of behavioral economics approaches}

Assuming limited structural barriers for the patient (e.g., high service fees), the most relevant decisions and actions relating to this behavior are done by the provider with minimal patient intermediation. This fact simplifies identifying relevant contextual cues driving this behavior. Behavioral economics approaches could cue the provider to consider this test, support employing the appropriate heuristics for determining when to screen, and facilitate acting on this intention to screen by eliminating hassles where feasible, among other tactics. Behavioral economics could also be used to strengthen how the provider frames the screening to the patient should patients be hesitant for reasons other than impactful financial constraints.

\section{Conclusion}

Behavioral economics approaches hold promise in increasing use of RDTs when structural barriers-stockouts in the case of the provider or high service fees in the case of the patient-are not the primary drivers of non-use in the context.

\section{Provider prescribes quality-assured artemis- inin-based combination therapy with correct dosage in cases of confirmed malaria}

\section{Potential link between provider behavior and health outcomes}

Appropriate treatment with artemisinin-based combination therapy (ACT) following a malaria diagnosis by RDT or microscopy is crucial to reducing malaria morbidity and mortality, limiting drug resistance from over-prescription, and ensuring that patients with other conditions are appropriately diagnosed and treated ${ }^{96}$ Stock-outs and shortages are common in low-resource settings, ${ }^{97}$ as are counterfeit ACTs, ${ }^{98}$ and these structural limitations may also influence providers' behavior. ${ }^{99}$ There may also be cost barriers, as antimalarials are not available free of 
charge to patients in all settings, and ACTs are generally more expensive than other antimalarial drugs. ${ }^{100}$

However, even when these structural barriers are not present, the literature notes various types of treatment error, including failure to prescribe any antimalarial after a positive test result, ${ }^{101,102}$ treatment with an antimalarial drug that is not recommended by best-practice guidelines or with an incorrect dosage, ${ }^{102,103}$ and prescribing an antimalarial after a negative test result because the patient presents with clinical symptoms of malaria or because the provider interprets the negative result as suspected malaria. ${ }^{104,105}$ Furthermore, providers may be susceptible to pressure from patients. In a qualitative study in Tanzania, clinicians reported feeling pressure from patients or fearing that they would complain if not given antimalarials, even though the patients did not directly request them. ${ }^{106}$ Another study in Kenya found that some providers are influenced by patients' preferences for certain types of medication..$^{99}$

Improving correct treatment after a malaria test thus has strong potential to improve outcomes by ensuring that patients with malaria are properly treated, improving diagnosis and treatment of other illnesses for which patients present with similar symptoms, and limiting drug resistance.

\section{Interventions focused on this behavior}

Training and job aids are common interventions to promote adherence to ACT prescription guidelines by providers, but they are often insufficient to generate improvements in treatment. For instance, having undergone training is not generally found to be associated with better prescription practice. ${ }^{107}$ The Integrated Management of Childhood Illnesses (IMCI) training approach, ${ }^{108,109}$ which involves on-site training, interactive learning, and opportunities for clinical practice, has shown some positive results for malaria treatment in Tanzania ${ }^{108}$ and Uganda. ${ }^{110}$

Job aids have been commonly employed as a complement to training, with mixed results. A study in Kenya found that the presence of a wall chart explaining correct prescription practices was significantly associated with better treatment quality. ${ }^{111}$ Other studies in Tanzania ${ }^{101,112}$ and Kenya ${ }^{113}$ found that training on ACT use and availability of job aids were not significantly associated with correct prescription by providers. A study in the Central African Republic found a negative association between presence of job aids and treatment quality. ${ }^{114}$
In Tanzania, a trial that tested interactive workshops on malaria testing and prescription, feedback summaries on RDTs and prescriptions, best practices and motivational messages delivered through SMS, and (for a subset) materials with Ministry of Health branding, found that these reduced providers' over-prescription of antimalarials to patients who had a negative RDT, but did not increase antimalarial prescription among those who had a positive RDT result. ${ }^{95}$

Improvements in knowledge or prescription practices from training tend to deteriorate over time. ${ }^{115}$ While the need for effective follow-up and supervision is commonly mentioned as an appropriate response, ${ }^{99,107,116}$ there is limited evidence of the effects of supervision on malaria treatment. A randomized evaluation in Nigeria found that supportive supervision using a checklist adapted from $\mathrm{IMCl}$ activities resulted in improvements in providers' knowledge of malaria and correct prescription practices. ${ }^{117}$ However, other studies have found that supervision visits were not significantly associated with correct prescription by providers in Tanzania ${ }^{101}$ and Malawi. ${ }^{118}$ In lieu of supervision, a study in rural Kenya found that text messages sent to health workers increased their adherence to proper malaria case management with medication by $23.7 \%{ }^{119}$

One review found that pre-packaging of drugs is one of the most effective ways of increasing adherence to dosage guidelines among both providers and patients. ${ }^{107}$ In Ghana, providers noted that when they received prepackaged antimalarial doses, they tended to modify their prescription behavior to dispense the correct packaged amount; patient compliance also improved with pre-packaging. ${ }^{120}$ In Burkina Faso, packaging antimalarials for children in age-specific packets with a full course of treatment led to improvements in adherence to dosage and duration guidelines for treatment. ${ }^{121}$

\section{Potential application of behavioral economics approaches}

In contexts where user fees are not a barrier, the most relevant decisions and actions are taken by the provider, but the patient may strongly influence the provider's behavior. In some contexts, over-prescription may be driven by patients' and their caretakers' demand for antimalarials. ${ }^{115,122}$ Behavioral economics approaches could be applied to support a clinician in framing their treatment recommendation in a way that is best positioned to be positively received. 
Behavioral economics can also be applied to support accurate decision-making and action in areas where contextual features unrelated to the patient may lead a provider to prescribe in a sub-optimal way. For instance, health workers from lower cadres tend to be more likely to prescribe ACT correctly. ${ }^{99,101,111}$ Behavioral economics could be applied to address potential overconfidence as it relates to identity to address this issue in higher-cadre health workers. There is also evidence that high patient load in facilities is associated with incorrect treatment practices; 99,123 behavioral economics could be applied to support providers in managing the negative impacts of scarcity (limited available mental bandwidth due in this case to excessive workload).

\section{Conclusion}

There is potential for behavioral economics approaches to improve provider ACT prescribing behavior when 1) accurate testing (RDT or microscopy) is available and used, 2) ACTs are free to patients, and 3) ACTs are consistently available in sufficient quantities so that rationing of drugs is not a rational provider response.

\subsection{Nutrition among children under two years old}

\section{Provider encourages breastfeeding within one hour after delivery}

\section{Potential link between provider behavior and health outcomes}

Breastfeeding within one hour after delivery provides many health benefits for the infant that are well-established in the literature. ${ }^{124,125}$ Encouraging early initiation of breastfeeding could accompany other essential newborn care in the case of facility-based births. While little research has considered providers' role in this behavior, a small number of observational studies has shown provider counseling ${ }^{126,127}$ and early skin-to-skin contact $^{128}$ (which can be facilitated by providers) to be associated with early initiation of breastfeeding. In one study in Madagascar, a multi-pronged intervention including provider-facing components was tested to increase breastfeeding initiation along with other outcomes. ${ }^{129}$ Among women who initiated breastfeeding within an hour, health workers and $\mathrm{CHW}$ s were by far the most common sources of information reported. ${ }^{129}$ The potential role for provider counseling is also suggested in literature identifying patient-side barriers. For example, one systematic literature review noted patients' cultural reasons for failing to initiate early breastfeeding, including the belief that colostrum is harmful ${ }^{130}$ or that administering other pre-lacteal feedings such as water or butter is beneficial. ${ }^{131}$ Provider counseling could help address such concerns, though this question does not appear to have been studied explicitly.

\section{Interventions focused on this behavior}

Training modules such as the WHO/UNICEF Breastfeeding Counselling: a training course attempt to equip providers with the skills to support mothers in early breastfeeding, such as through observing and assessing breastfeeds and asking open questions and empathizing during counseling. ${ }^{132}$ In an RCT conducted in Brazil, those who received the training experienced statistically significant increases in knowledge as well as clinical and counseling skills (though patient breastfeeding behavior was not assessed). ${ }^{133}$

A more comprehensive approach with patient and provider-facing components is embodied in baby-friendly hospital support. This support consists of facility-level practices under the rubric of " 10 Steps to Successful Breastfeeding." Recommended steps include having a written breastfeeding policy communicated to all staff, training staff in the skills necessary to implement the policy, informing pregnant women about the benefits of breastfeeding, helping women initiate breastfeeding within a half-hour after birth (for example by demonstrating how to position the baby and achieve a latch), and showing mothers how to maintain lactation. A recent systematic review and meta-analysis found that Baby Friendly Hospital support resulted in a $20 \%$ increase in early initiation of breastfeeding. ${ }^{134}$

Another provider-focused intervention mentioned earlier in the review is the use of checklists. WHO's SCC includes a point around initiation of breastfeeding and skin-to-skin contact in the "soon after birth" section of the list to make this action more salient for the provider. A recent evaluation of the SCC in India saw moderate absolute increases in breastfeeding within one hour of birth in facilities implementing the SCC with coaching. ${ }^{135}$

\section{Potential application of behavioral economics approaches}

Both providers and patients play a role in ensuring prompt initiation of breastfeeding, but unlike in several other behaviors, the actions of both occur within a narrow timeframe and in the same physical context. While 
structural barriers such as limited staffing in facilities may make it more difficult for providers to counsel consistently, ${ }^{8,15}$ there may be behavioral dimensions of these barriers as well and ways to optimize the provider environment to increase counseling. Behavioral economics approaches could be used to cue a provider to counsel and strengthen how to frame the messages in a way that better addresses patient concerns. Behavioral economics approaches could be used to prime patients to consider breastfeeding initiation, which would then facilitate effective provider counseling-important given providers' limited time and workload. Since this action is likely to occur within the health facility, contextual cues that could prompt the right decisions and actions in providers and patients may be more easily identified and addressed with behavioral economics approaches.

\section{Conclusion}

Behavioral economics approaches hold promise in increasing provider counseling in initiation of breastfeeding within one hour of delivery in contexts where facility-based delivery is high. It is important to note that having a high facility-based delivery rate is not a contextual limitation but simply a high-impact context. Behavioral economics approaches can support this goal for the women who deliver in facilities regardless of the general prevalence of facility-based delivery in the context.

\section{Provider encourages exclusive breastfeeding for six months}

\section{Potential link between provider behavior and health outcomes}

Exclusive breastfeeding for the first six months of life confers health benefits for infants. Besides nutrition, these benefits include preventing diarrhea and other common childhood illnesses. ${ }^{136}$ Evidence shows that providers may be effective in promoting duration of exclusive breastfeeding, especially with several interactions with patients (e.g., three to 6) occurring across both the prenatal and postpartum periods; a literature review suggests these are both important moments to provide support as long as the interventions are only moderately time-intensive. ${ }^{137}$ Studies conducted in Mexico, Uganda, Burkina Faso, and South Africa with peer counselors and in Ghana with nurse counselors also found that rates of exclusive breastfeeding were higher among women who had received counseling and frequent follow-up visits from counselors. ${ }^{138-140}$ Combined individual and group education and counseling, whether conducted at the facility or in the community by health professionals or lay counselors, may also increase duration of breastfeeding. ${ }^{141}$ Health care providers may be most effective when they understand the sociodemographic and cultural determinants of exclusive breastfeeding, such as perceived insufficient milk supply or beliefs about infant nutrition, so that they can offer practical guidance in advance to address these barriers. ${ }^{142}$ It should be noted, however, that some barriers to exclusive breastfeeding may be structural and not easily overcome with provider intervention. In a systematic review of exclusive breastfeeding in lower-resource settings, maternal employment or illness were among the most common non-behavioral barriers to exclusive breastfeeding. ${ }^{142}$

\section{Interventions focused on this behavior}

A study in Madagascar indicates that provider training was effective in increasing exclusive breastfeeding when combined with additional messaging and community mobilization. In this instance, 1- to 2-day skills-based modules for providers and CHWs focused on reviewing protocols for contact with clients and enhancing interpersonal communication skills to encourage mothers to try a new practice. These elements were combined with community mobilization events and dissemination of key messages through local mass media and resulted in an increase in the rate of exclusive breastfeeding of infants under six months of age. ${ }^{143}$

This finding is consistent with other literature indicating that training of hospital staff, when integrated with mass media, counseling, and community mobilization significantly increase exclusive breastfeeding prevalence. ${ }^{129,134}$

Job aids, such as counseling cards, have also been used to support health workers in encouraging exclusive breastfeeding. ${ }^{150}$ These cards were part of a broader intervention tested in Benin, which also included training through role-playing and technical reference guides. The intervention was shown to increase the exposure of pregnant women to messaging around breastfeeding; however, exclusive breastfeeding itself was not measured. ${ }^{144}$

\section{Potential application of behavioral economics approaches}

Both providers and patients are involved in making relevant decisions and taking action related to this behavior. For patients who have not decided whether to undertake exclusive breastfeeding or who have decided not to breastfeed, a provider may initiate this conversation to try to address concerns. Behavioral economics 
approaches could be used to cue a provider to have this conversation with targeted framing during a number of potential touchpoints including ANC, PNC, or vaccination. A patient could then decide to exclusively breastfeed but would need to take continuous action on this intention. Should an appropriately timed touchpoint exist, a provider could be cued to address concerns that arise after the patient takes the initial action. Importantly, as noted in the literature, frequent follow-ups by providers may be critical to support patients in overcoming potential behavioral barriers to maintain the behavior. This strategy itself could be devised using theory from behavioral economics, and the content of these follow-up sessions could also be optimized with behavioral economics.

\section{Conclusion}

Behavioral economics approaches focused on providers may hold promise for increasing exclusive breastfeeding when 1) ANC, PNC, facility-based delivery, or vaccine coverage is high and there are frequent interactions with the health system during the 6 -month postpartum period or 2) there is a well-organized cadre of CHWs trained in exclusive breastfeeding counseling with strong postpartum identification and frequent contact with new mothers but adherence to counseling is low.

\section{Provider counsels on minimum meal frequency and nutritious, age-appropriate meals}

\section{Potential link between provider behavior and health outcomes}

Complementary feeding, or introducing solid foods to infants over six months (with or without continued breastfeeding), supports children's growth and health, although guidelines on appropriate timing and composition of foods vary across countries. ${ }^{145}$ The role of providers may be significant in this domain, despite inconsistent guidelines. A meta-analysis including 17 randomized and quasi-randomized studies showed that nutrition counseling alone as well as the provision of complementary foods led to significant increases in the weight of infants six months to two years old, possibly resulting from greater food diversity (including more animal products), higher meal frequency, and better food-related hygiene. ${ }^{146-148}$ Most interventions involved community health or nutrition educators conducting home visits or group trainings, ${ }^{148-151}$ whereas fewer enlisted doctors. ${ }^{152}$

\section{Interventions focused on this behavior}

Provider training has been conducted to spur provider counseling. The WHO course Complementary Feeding:
Family Foods for Breastfed Children forms the basis of a 3-day training course for health professionals on the topic. This course was evaluated in an RCT in Egypt, which found better outcomes in the treatment versus control group: Clinicians were more likely to conduct counseling and give appropriate advice, mothers had higher recall of advice, and children had statistically significantly greater weight gain. ${ }^{153}$

The WHO Global Strategy for Infant and Young Child Feeding suggests the role of providers and lay counselors to increase counseling. ${ }^{154} \mathrm{~A}$ review of studies of in-service provider training on child nutrition found that training led to improvements in providers' knowledge (18 of 18 studies) and counseling (12 of 12 studies)-including counseling skills, likelihood of providing nutrition advice, and appropriateness of the advice given. ${ }^{155}$ Studies in Brazil ${ }^{152,156}$ and Pakistan ${ }^{157}$ found that training providers on child feeding counseling improved providers' communication skills during counseling with mothers of sick children, improved mothers' knowledge of recommendations, and reduced growth faltering in children.

Quality improvement interventions can also improve provider counseling on nutrition. A study in clinics in Guinea and Kenya found that COPE services led to improvements in counseling and respectful care in a number of areas, including child nutrition. The approach was intended as a complement to the ICMI training and consisted of self-administered guides encouraging providers to consider and respond to issues of service quality, accompanied by supportive supervision. ${ }^{61}$

Job aids such as pictorial books to accompany provider counseling and materials for facilitating community meetings on infant nutrition have been produced by many organizations. However, studies examining the efficacy of such interventions are sparse.

\section{Potential application of behavioral economics approaches}

Both providers and patients are involved in making relevant decisions and taking action related to this behavior. First, a provider such as a CHW would initiate the counseling either cued by a patient or conducted per standard practice. Behavioral economics approaches could be used to make this action top of mind for the provider should salience be a barrier. Behavioral economics could also be used to optimize the counseling itself, whether it be to structure the content in a way that helps avoid choice overload, reframe the messages so they address 
client concerns to spur action, or simplify the delivery of the counseling itself, among other options depending on the barriers at play. A patient would then need to make a decision about this advice and take action on it. In optimizing the counseling with behavioral economics approaches, attention could be paid to ensuring that initial patient intention is established during the counseling session itself, so that the provider angle can be most effective. A range of different patient-side barriers might exist, which could arise from the moment of intention formation with the provider to the practice of these behaviors in the household by the patient. Frequent interaction with the health system in this period can help ensure that the provider will be well-positioned to support patients in taking action. Behavioral economics approaches could be used to cue community-based or facility-based providers to engage in conversations with patients about feeding practices after the initial counseling and could help optimize provider-oriented interventions to support maintenance of these patient behaviors.

\section{Conclusion}

Behavioral economics approaches focused on providers may hold promise for strengthening provider counseling on meal frequency and content when 1) immunization coverage is high and providers trained in meal frequency and content are available during these sessions, or 2) there is a well-organized cadre of CHWs trained in meal frequency and content who have frequent contact with new mothers but adherence to counseling is low.

\subsection{HIV/AIDS testing and treatment adherence}

\section{Provider initiates testing and counseling in a health facility}

\section{Potential link between provider behavior and health outcomes}

It is well-established in the literature that provider-initiated testing and counseling (PITC) increases HIV testing uptake $\mathrm{e}^{158}$ and is often the main entry point to comprehensive HIV care for anyone who interacts with a provider. ${ }^{159}$ Since PITC can occur regardless of whether or not patients have HIV-related signs or symptoms, this behavior has particularly high potential to affect health outcomes by helping providers identify HIV-positive patients who would not have otherwise sought testing-or been recommended testing - and can reap the benefits of earlier treatment initiation.
Moreover, the way in which providers offer testing can also influence testing uptake and subsequent health outcomes. There is compelling evidence that offering HIV testing as a routine step that patients are expected though not required to undergo (an opt-out rather than an opt-in approach) can increase testing uptake, ${ }^{160,161}$ in part by reducing perceived stigma associated with testing and making it easier for both providers and patients to discuss HIV openly. ${ }^{162,163}$

Although PITC has been recommended by the WHO since $2007^{159}$ and commonly forms a component of countries' HIV testing guidelines, ${ }^{164}$ gaps in provider compliance remain. ${ }^{165-167}$ Structural barriers such as stock-outs and shortages of testing supplies, understaffed facilities, and lack of private spaces to deliver counseling sometimes hinder delivery of PITC, ${ }^{165,168,169}$ although there is also evidence that behavioral barriers (discussed below) are at play.

\section{Interventions focused on this behavior}

The WHO recommends that all providers who perform testing receive training and ongoing supervision on obtaining informed consent, protecting confidentiality and privacy, avoiding stigma and discrimination, and conducting appropriate referral. ${ }^{159}$ The WHO has compiled an extensive library of training tools and materials for providers on these topics. ${ }^{170}$ In interventions introducing routine opt-out testing in ANC settings (which led to substantial increases in testing uptake), clinic staff received training on how to introduce HIV testing as part of routine antenatal services and to dispel the common misconception that testing is mandatory, though these studies did not evaluate the effectiveness of the training itself on increasing screening rates. ${ }^{160-162}$

An impact evaluation of Rwanda's pay-for-performance scheme found that financial incentives to providers increased HIV testing and counseling. Providers were paid based on a range of outcomes, including number of clients tested and number of couples tested, with a significantly higher payment for couples. The likelihood of an individual being tested increased by $6.1-10.6 \%$ while the likelihood of a couple being tested increased by $10.2-14.5 \% .{ }^{171}$

Provider-oriented interventions have also focused on reducing the effects of stigma in HIV testing and treatment. The Horizons Program evaluated 10 such programs across several regions, finding that multi-pronged interventions led to reductions in biased treatment, 
improvements in understanding and equitable use of universal precautions, and improvements in counseling and testing practices such as seeking informed consent. Rates of provider-initiated testing were not measured. ${ }^{172}$

One component of this program was sharing data on stigma and its effects on the health care facility to help facilities and providers recognize and acknowledge its existence, which researchers found led to improved buy-in and engagement from management. In India, this data was used to develop a supervisory checklist to help hospital managers identify discriminatory practices. Involving management in this way helped catalyze reform in the facilities. ${ }^{173} \mathrm{~A}$ pre-post analysis of another component in Vietnam found that the introduction of "safe and friendly" hospital policies covering issues such as access to testing and confidentiality led to reductions in discriminatory practices. ${ }^{174}$ Another component of the multi-pronged approach included trainings to all cadres of providers. A study in Vietnam found that the addition of a workshop, co-facilitated by people living with HIV, on the causes and manifestations of social stigma, led to greater improvements in testing and treatment behaviors than a standard training on HIV care alone. ${ }^{172}$

\section{Potential application of behavioral economics approaches}

In order to complete this behavior, providers must remember to initiate testing, decide to initiate testing for a particular patient, act on that decision, and offer testing in the correct manner and accompanied by appropriate counseling. Behavioral economics approaches could be used to address the provider barriers that may arise at these stages to increase compliance with PITC. For example, behavioral economics approaches could remind providers to test patients and enhance provider motivation. ${ }^{165}$ They could also help mitigate discomfort or stress associated with testing ${ }^{175}$ and fear of confronting an HIV-positive test result. ${ }^{169}$ Where provider attitudes toward patients affect PITC, from deterring high-risk patients from coming in for testing ${ }^{172}$ to directly denying care, ${ }^{176}$ behavioral economics approaches could help shift patterns of behavior and mental models.

\section{Conclusion}

Provider-focused behavioral economics approaches hold promise for improving provider adherence to PITC when testing supplies are available.

\section{Provider delivers community-based testing ser- vices according to best practices}

\section{Potential link between provider behavior and health outcomes}

Community-based testing services, often delivered by trained lay providers, are a key step in identifying HIV-positive individuals and linking them to appropriate treatment, and in particular to reaching key populations that are unlikely to visit a health care facility or to proactively seek out testing services. ${ }^{164}$ Testing strategies outside the facility setting have increased uptake, as providers can influence who receives facilitated access to testing services and is therefore more likely to be tested. ${ }^{177,178}$ Providers' delivery of community-based testing services can also influence health outcomes through their choices about where and how to focus their efforts. For example, the WHO recommends index testing as a strategy to improve efficiency and cost-effectiveness of HIV testing services, ${ }^{164}$ though providers may resist or struggle with this shift in testing approach. ${ }^{179}$

Structural barriers such as a lack of supplies and equipment for testing, long distances to reach patients, and limited availability of trained providers to conduct testing pose a challenge to delivering testing services. In the absence of these barriers, provider behavior can influence health outcomes by increasing coverage of testing services and ensuring that testing reaches the populations most likely to benefit from it.

\section{Interventions focused on this behavior}

While there is evidence suggesting that community-based testing through CHWs and other providers can enhance uptake and quality of HIV services, there is scant evidence on related provider-facing interventions in this area. ${ }^{178}$ In Malawi, CHWs received two weeks of theoretical training on care and support for both HIV/AIDS and tuberculosis, plus on-the-job training with community nurses. ${ }^{180}$ However, these interventions were not evaluated to measure their effectiveness in improving community-based testing.

Interventions aimed at shifting provider testing strategies have successfully increased rates of index testing, but gaps in compliance remain. The Clinical and Community HIV/AIDS Services Strengthening project in Mozambique trained community counselors on index testing and 
implemented a data monitoring system to track counselor performance. Monitoring allowed for follow-up with specific counselors for additional skill-building to improve results, as well as to to address barriers identified among certain counselors who failed to switch to index testing. ${ }^{179}$

\section{Potential application of behavioral economics approach}

Both provider-facing and patient-facing interventions may be effective in increasing coverage of HIV testing among target populations. Behavioral economics interventions among providers performing community-based outreach and testing could increase compliance with defined testing policies (such as index testing or targeting key populations) by strengthening the intention to follow guidelines and supporting follow-through on this intention. ${ }^{179}$ Where guidelines allow discretion on the part of providers as to where they focus testing outreach, behavioral economics approaches could be used to support targeting testing with appropriate heuristics for identifying high-risk groups and salient feedback on outreach efforts. Behavioral economics could also be used to support providers in how they offer testing services, for example framing testing services in a way that most resonates with at-risk, under-tested groups and organizing these campaigns in a way best positioned to help patients overcome their concerns or address potential provider stigma. ${ }^{181}$

In cases where front-line providers have less control over who is offered testing, or where target populations are already being offered but are not accepting testing services, patient-facing behavioral economics interventions may hold more promise instead of (or as a complement to) provider-facing approaches.

\section{Conclusion}

Provider-focused behavioral economics approaches hold promise for improving testing and counseling in community-based settings when testing supplies are available and providers are adequately trained.

\section{Provider ensures accurate testing}

\section{Potential link between provider behavior and health outcomes}

Accurate testing is essential to ensuring timely diagnosis and treatment for HIV-positive individuals and as a safeguard against initiating treatment unnecessarily for HIV-negative individuals whose initial test result is reactive. Accurate testing relies on adherence to the relevant testing algorithm, which specifies the correct type and sequencing of tests to use under different circumstances and how different types of results should be interpreted. ${ }^{182}$ Although RDTs are relatively simple to perform, providers can make errors in interpreting results that identify false positives (HIV-negative individuals incorrectly started on ART) and false negatives (HIV-positive individuals who are not referred for treatment). ${ }^{183}$ Depending on test results and recent or ongoing risk of exposure, patients should be re-tested within a specified timeframe or should receive additional confirmatory testing. ${ }^{164}$

The appropriateness of a provider's counseling in each of these scenarios and how the provider manages the conversation with a patient may also influence the likelihood of the patient's return for subsequent re-testing and confirmatory testing, increasing diagnosis rates. Additionally, some providers may be able to mobilize outreach efforts in the case that a patient does not return, which can also impact accurate testing. Accurate testing, in turn, is needed to ensure that HIV-positive individuals receive the appropriate treatment (see Provider initiates appropriate HIV care immediately after a diagnosis is confirmed, page 30) while minimizing the potential cost and unnecessary harm from initiating ART in patients who are not in fact HIV positive. ${ }^{184}$

\section{Interventions focused on this behavior}

In low-resource settings, most HIV testing is performed by non-laboratory providers, for whom the CDC and WHO have developed a training package as well as guidelines for countries to roll out a training program. ${ }^{183}$ Non-laboratory providers can provide testing with a high degree of accuracy even after a brief training; in Cambodia, non-laboratory providers who underwent a half-day training performed marginally better than laboratory staff. $^{185}$

The WHO's training recommendations place particular emphasis on the importance of training and supportive supervision for lay providers. ${ }^{164}$ A recent review found that after completing a training program, lay providers are generally able to collect and interpret diagnostic tests with comparable accuracy to a clinician and are acceptable to patients, though most studies did not assess the accuracy or quality of the counseling provided by lay providers. ${ }^{186}$ Moreover, these studies did not evaluate the type or extent of training needed to support accurate testing, though even brief trainings have been 
shown to improve quality. A study in Zambia found that participation in the 3-day national training program on HIV rapid testing was significantly associated with testing accuracy. ${ }^{187}$

In addition to training, quality assurance programs consisting of on-site supervision, standardization of trainings and tools - such as a log book for use at all testing sites-and proficiency testing are recommended to sustain results from training. ${ }^{183,188}$ Proficiency testing consists of sending simulated samples to HIV-testing sites to assess the accuracy of results and evaluate technical competence in testing, and can help identify gaps in provider capacity and further training needs. ${ }^{187,189}$

In Zambia, a standards-based management and recognition program involved detailed performance standards outlined in a checklist used to assess provider performance, combined with training, supportive supervision, and on-site assistance. The program led to a $36 \%$ increase in repeat HIV testing during ANC (in accordance with prevention of mother-to-child transmission [PMTCT] guidelines), as well as improvements in ongoing counseling on HIV testing during ANC. ${ }^{190}$

\section{Potential application of behavioral economics approach}

Most actions needed to ensure accurate testing lie with the provider. Behavioral economics approaches may hold potential to reinforce proper processes and interpretation in busy work environments or where repetition dampens providers' adherence to the testing algorithm. In addition, behavioral economics could inform interventions to build providers' confidence when they have not received extensive training but still hold the requisite skills. In other settings, prompts for providers could help mitigate overconfidence in initial test results or clinical assessments. Behavioral economics can also offer strategies to help providers deliver uncomfortable and often unwelcome news about necessary follow-up to patients in a way that is both clear and motivating.

\section{Conclusion}

Behavioral economics approaches have potential to support accurate testing, including effective counseling to clients on retesting or confirmatory testing, where testing supplies are available and where providers receive adequate training on the mechanics of performing and interpreting tests.

\section{Provider performs voluntary assisted partner notification services}

\section{Potential link between provider behavior and health outcomes}

Disclosure of positive HIV test results to a patient's sexual or drug-use partners has potential to improve health outcomes in two ways: by identifying individuals with an increased likelihood of being HIV positive and linking them to testing and treatment ${ }^{191,192}$ and by supporting effective preventive steps for couples in serodiscordant (mixed HIV status) partnerships. Providers can play a role in partner notification in several ways. Passive notification occurs when a provider encourages HIV-positive individuals to disclose their status to partners. Provider referral occurs when, with the consent of the HIV-positive patient, the provider confidentially contacts the person's partner directly to offer testing services. In contract referral, HIV-positive patients enter into a contract with a provider and agree to disclose their status and potential HIV exposure to their partners within a specific time period. If the partners of the HIV-positive individual do not access testing or contact the provider within that period, the provider will contact the partners directly. In dual referral, the provider accompanies the patient when they disclose to partners. Provider, contract, and dual referral are methods of assisted partner notification recommended by the $\mathrm{WHO}{ }^{193}$

Providers play an essential role in assisted partner notification, both by counseling HIV-positive patients on options for notifying partners and by accompanying patients when they disclose to their partners or by following up directly with partners. Assisted partner notification has been shown to increase screening rates and detection of HIV among partners. ${ }^{194}$ Moreover, the manner in which a provider offers voluntary assisted partner notification to HIV-positive individuals may influence the patient's willingness to disclose and help minimize any associated risks to the disclosing partner. ${ }^{193}$

\section{Interventions focused on this behavior}

The WHO highlights the importance of training and support for providers counseling patients appropriately on partner notification, reinforcing the voluntary nature of partner notification, tracing and locating partners, documentation and reporting, risk minimization, and maintaining privacy. ${ }^{193}$ However, we are not aware of any evidence on the relative effectiveness of different types of training, supervision, or other support for providers on 
partner notification or any other interventions that are meant to change provider behavior with regard to this practice in particular.

\section{Potential application of behavioral economics approach}

To carry out assisted partner notification, the provider must first discuss patients' HIV status and sexual and drug-use history and counsel on disclosure options. The provider is tasked with convincing but not pressuring patients to disclose, a balance that behavioral economics approaches could help strike through framing and motivation. Although there are often barriers on the patient side to consenting to partner notification (including fear of stigma or violence), providers may play an important role in helping patients overcome those barriers.

If the patient consents to assisted notification, providers must carry out their role in notification by accompanying the patient when they disclose to partners or by notifying partners directly. Where structural barriers do not impede notification, behavioral economics could help providers to overcome procrastination and competing claims on their attention and to conduct ultimate disclosure conversations sensitively in a way that encourages appropriate care.

\section{Conclusion}

Behavioral economics approaches may hold promise in increasing partner disclosure and testing when adequate testing supplies are available and assisted HIV partner notification services are recommended by national or local policy but partner disclosure and testing remain low.

\section{Provider initiates appropriate HIV care immedi- ately after a diagnosis is confirmed}

\section{Potential link between provider behavior and health outcomes}

HIV testing can only serve as a gateway to treatment if providers link patients to appropriate, timely care after diagnosis. This includes initiating ART as soon as possible for everyone living with HIV regardless of CD4 count (which indicates the effect of the virus on a person's immune system). This "Test and Start" approach improves health outcomes for the HIV-positive patient and reduces the likelihood of transmission. ${ }^{195}$ For providers who are authorized to prescribe ART and work in facilities that stock it, this means prescribing ART immediately upon confirmation of the diagnosis. For other providers, it means referring the patient to a facility where they can begin ART and supporting the patient to ensure follow-through (in some cases, accompanying the patient to the facility where they can receive ART). ${ }^{196}$

In either case, appropriate immediate care upon diagnosis also includes counseling patients on the meaning of their diagnosis and the importance of taking and adhering to ART. ${ }^{197}$ There are potential negative impacts of beginning and not maintaining treatment, including increased infectiousness ${ }^{198}$ and development of drug resistance. ${ }^{195}$ WHO recommendations nonetheless conclude that the benefits of early treatment initiation outweigh the potential risks, though these risks underscore the importance of effective counseling beginning from the time of diagnosis, and in some cases ongoing adherence support, to accompany the Test and Start approach. ${ }^{195}$

HIV-positive patients often fail to initiate treatment when they are eligible. ${ }^{199,200}$ Although structural barriers, including poor information systems, lack of available drugs, and other direct and indirect costs are often at play, ${ }^{201}$ the evidence suggests that providers can play a role in strengthening the linkage to treatment. ${ }^{167,196}$ Improving linkage to care and preventing loss to follow-up is estimated to nearly double the benefits (reduced death toll and prevention of new infections) from universal testing and treatment. ${ }^{202}$

\section{Interventions focused on this behavior}

Referral systems for HIV care are often weak and fail to generate feedback to providers on who has followed up and when, ${ }^{199}$ and developing more robust referral systems with tracking tools has been shown to support providers to facilitate referrals more effectively. In a study in Tanzania, 2-part referral forms with unique matching numbers were used both to facilitate patients' access to the HIV clinic and to monitor the proportion of diagnosed clients who followed up. When combined with other patient-facing interventions, referral rates were near $100 \%$, with more than two-thirds of patients following through on the referral.

Training and supportive supervision have been shown to support effective initiation of ART by lower cadres of providers. The Streamlining Tasks \& Roles to Expand Treatment and Care for HIV (STRETCH) program in South Africa combined educational outreach to nurses with supervision by a facility manager and a toolkit (including a checklist outlining the six key elements of 
comprehensive HIV care to be integrated into all primary care services and letters authorizing nurses to prescribe ART). ${ }^{203}$ The nurses were able to safely deliver ART and the intervention improved several health outcomes and quality-of-care indicators but did not reduce mortality rates. ${ }^{204}$ Conversely, brief trainings of a few days were found in Uganda and Zimbabwe to be insufficient for nurses to feel comfortable managing ART for PMTCT and were supplemented with ongoing supervision. ${ }^{205}$

A randomized trial in South Africa found that an on-site outreach education program for all clinic staff, as compared with offsite training for ART specialist nurses only, ${ }^{206}$ improved health workers' compliance with recommended care following an HIV diagnosis. ${ }^{a}$ The outreach education program covered practical topics such as coordination between providers in the facility and considerations of the clinical context and simplified material from multiple sources into a single set of guidelines. ${ }^{206}$ An online distance learning program was found (in a pre-post analysis) to improve providers' knowledge of ART and their reported confidence initiating ART and encouraging patient adherence. ${ }^{207}$

A study in Malawi found that a simple screening tool for ART eligibility for CHWs outperformed the then-current WHO clinical staging guidelines for determining ART eligibility. The tool allowed providers to classify patients as ART-eligible based on a small number of readily observable features and was far less complex and time-consuming than WHO staging. ${ }^{208}$ While this particular tool will become less relevant with the expansion of universal HIV treatment regardless of CD4 count, its effectiveness suggests that streamlined or simplified versions of referral or prescription guidance may be effective in supporting appropriate HIV care initiation, especially for lower provider cadres.

\section{Potential application of behavioral economics approach}

A provider undertakes most of the necessary decisions and actions in providing HIV care after diagnosing a patient. Behavioral economics approaches may be useful in navigating complex and evolving standards of care, boosting providers' confidence in their skills and judgment when they are adequately trained. Behavioral economics could also inform efforts to ensure providers emphasize treatment for all patients, as some evidence

an that study, provision of co-trimoxazole prophylaxis before ART initiation. indicates that providers may hesitate to prescribe ART to patients they think are unlikely to adhere to treatment. ${ }^{209}$ Beyond offering services to all patients, counseling and encouraging could be further behaviorally optimized to address barriers patients may face at this stage.

\section{Conclusion}

There is potential for behavioral economics approaches to improve immediate ART initiation where 1) ART is available and 2) the main barriers to treatment for patients are not structural.

\section{Provider regularly monitors viral loads}

\section{Potential link between provider behavior and health outcomes}

Regular monitoring of individuals on ART is important to ensure treatment efficacy and improved health outcomes. ${ }^{210}$ Monitoring allows providers to identify cases in which the patient is not adhering to ART or has developed drug resistance and to adjust ART or provide additional support accordingly. ${ }^{211}$ Correct monitoring involves both frequency of monitoring ${ }^{\mathrm{b}}$ and use of the most appropriate method. Where available, viral load monitoring is the recommended method because it detects treatment failure earlier than CD4 count monitoring or clinical symptoms, often before resistance has developed. ${ }^{211}$

Point-of-care (POC) viral load testing is beginning to become available in resource-limited settings. ${ }^{212}$ However, testing is still frequently done by sending samples to a laboratory, in some cases with waits of several weeks or longer for results. ${ }^{213}$ As a result, performing viral load monitoring requires providers to identify patients who are due for monitoring, stimulate them to undergo monitoring, take samples correctly, send them to a laboratory, and receive, read, and document results. This set of provider behaviors is essential to communicating results to patients and providing timely support to patients whose viral loads are not ideal (see Provider communicates results of monitoring to patients, page 32 and Provider counsels if viral loads are not ideal, page 33). These behaviors have great potential to influence health outcomes by increasing the number of HIV-positive individuals who have achieved viral load suppression ${ }^{214}$ and limiting the development of drug resistance. ${ }^{215}$

${ }^{b} \mathrm{WHO}$ guidance recommends monitoring at 6 and 12 months after initiating ART and every 12 months thereafter.. ${ }^{210}$ 
Structural barriers (including high cost, lack of transportation, and insufficient equipment) limit access to viral load monitoring in many contexts. ${ }^{216}$ However, provider behavioral barriers such as improper processes and equipment use ${ }^{217}$ as well as incomplete understanding of the benefits of viral load monitoring also all contribute to under-use, ${ }^{217}$ suggesting opportunities for behaviorally informed interventions.

\section{Interventions focused on this behavior}

Viral load monitoring has until recently been unavailable in most low- and middle-income countries. As a result, although recommendations for promoting provider adherence exist, few studies to date have documented any evidence of their effectiveness in promoting provider behaviors.

A review of several interventions to support scale-up of viral load testing suggests that provider training and use of monitoring and evaluation tools to identify and correct gaps support scale-up efforts. ${ }^{218}$ WHO guidelines recommend training and supportive supervision for clinicians, including clear messages for providers on the rationale for viral load testing as well as guidance on viral load clinical algorithms, interpreting results, and documenting follow-up. ${ }^{219}$

In Botswana, providers significantly increased correct CD4 and viral load monitoring when a clinical mentorship program was implemented. 220

A study in Mozambique found suggestive evidence that use of the iDART system-a pharmacy software that facilitates ART stock control, dispenses drugs, monitors patients, sends messages to patients who are late to treatment, and flags patients with a high viral loadhelped improve adherence to viral load monitoring by improving communication with patients who required enhanced adherence counseling. ${ }^{221}$

Médecins Sans Frontières (MSF) has implemented several interventions to improve viral load monitoring, although none have been evaluated. In one intervention, a formally assigned staff member serves as a "viral load focal point" dedicated to managing documentation and identifying patients in need of adherence counseling and repeat viral load testing. In Zimbabwe and Lesotho, clinics set monthly targets for viral load tests and mentors provided regular feedback. MSF suggests these targets facilitated supervision and enhanced motivation. In Zimbabwe, viral load testing was incorporated into patients' regular ART appointments and instructions around when to test were simplified to facilitate appropriate test timing. While not evaluated, this intervention reflected experiences in Malawi, where restrictive instructions around viral load testing resulted in patients not being tested. 222

\section{Potential application of behavioral economics approaches}

Effective viral load monitoring relies on actions undertaken by both patients and providers. Monitoring is predicated on a touchpoint with patients. As such, behavioral economics could inform demand-generation efforts to encourage HIV-positive patients to visit facilities for testing 222 or identification and outreach through community-based providers. Among providers, behavioral economics could inform trainings to familiarize providers with new technologies they may not fully understand (even if they have been adequately trained). ${ }^{217}$ Behavioral economics could also help overstretched providers ${ }^{221}$ comply with a number of additional complex steps or adopt new guidelines in a timely fashion. Finally, behavioral economics approaches could help temper providers' overconfidence in their ability to conduct accurate clinical monitoring, since their assessments of patients' status and adherence to ART tend to be inaccurate. ${ }^{223}$

\section{Conclusion}

There is potential for behavioral economics approaches to improve provider compliance with viral load monitoring guidelines where 1) viral load testing technology is available and there are no major structural barriers to its use, 2) providers are adequately trained in the technology but use it insufficiently or incorrectly, and 3) adherence support or second-line ART (for treating drug-resistant cases) is available for cases in which treatment failure is detected.

\section{Provider communicates results of monitoring to patients}

\section{Potential link between provider behavior and health outcomes}

A critical component of regular monitoring is communicating the results of the test and the importance of viral load monitoring to patients. ${ }^{224,225}$ This may be accomplished through a follow-up visit to the health facility, text messages, or outreach by providers (including volunteers and other community-based providers) to patients after results are received. Alternatively, where samples are processed on-site, results can be communicated 
on the same day. ${ }^{226}$ In the case that viral loads are high, this communication is critically important to ensure that patients do not become despondent and that they receive the appropriate support (see Provider counsels if viral loads are not ideal, next section). ${ }^{227}$ For patients who have suppressed viral loads, communicating results of monitoring appropriately can serve as a positive affirmation of treatment, ${ }^{227}$ which could improve health outcomes if the affirmation increases patients' motivation to maintain adherence to treatment.

There is evidence that a large proportion of patients with elevated viral load results do not receive repeat viral load testing, suggesting that providers may not be communicating results or their implications effectively. ${ }^{228}$ Structural barriers such as difficulty tracing or contacting patients, poor sample management, and limited staff capacity impede viral load monitoring follow-up with patients. ${ }^{216,229}$ However, these impediments are not significant enough to rule out any contribution behavioral economics might make to improve the consistency and quality of communicating monitoring results.

\section{Interventions focused on this behavior}

As in the case of conducting viral load monitoring, recommendations for promoting follow-up results communication exist; however, we were not able to find studies documenting evidence of their effectiveness in promoting provider behaviors.

MSF recommends a clearly defined process for follow-up and a point person assigned to review, document, and act on results to improve timely follow-up. These recommendations are based on programmatic or laboratory scale-up of viral load testing in seven countries between 2013 and 2016. In Zimbabwe and Malawi, a high viral load form was used to flag when follow-up is required and also to facilitate outreach and communication to patients. ${ }^{222}$ SMS communication to clinics and patients served as an additional means of contacting patients. ${ }^{222}$ While MSF's implementation suggests these strategies could hold promise, they were not rigorously evaluated.

\section{Potential application of behavioral economics approaches}

A provider's role in communicating the results of monitoring depends on the provider's cadre and position and the health care setting itself. Where patients proactively visit a facility to receive viral load results, the provider's role may be limited to appropriately counseling patients during testing on the importance of follow-up. In this case, it is likely that most behavioral barriers occur on the patient side; thus, patient-facing interventions may hold the most promise in those settings. However, where providers are equipped to perform patient outreach to communicate results or encourage follow-up, behavioral economics may be applied to these processes to improve outcomes. For instance, behavioral economics could help instill an intention to act by dispelling assumptions about patients who are unlikely to be helped by these measures. Behavioral economics could also be used to provide a clear moment at which outreach is expected to occur or a deadline for performing it, especially when tracking and communicating with patients may require persistence and sustained attention.

\section{Conclusion}

There is potential for behavioral economics approaches to increase and improve provider communication of viral load results to patients where 1) viral load results are consistently received by the facility and 2) there are existing cadres of community-based providers to conduct outreach.

\section{Provider counsels if viral loads are not ideal}

\section{Potential link between provider behavior and health outcomes}

Routine monitoring can detect cases of non-adherence, but improving adherence (and re-suppressing viral loads) necessitates following up with patients if their viral loads are not ideal and linking them to adherence support services or providing those services directly. This step is critically important to ensuring that monitoring translates to improved health outcomes. The WHO recommends an adherence-support intervention followed by repeat viral load testing for all patients with high viral loads. ${ }^{219}$

In low-resource settings, adherence counseling is often conducted by lay providers and takes place in either individual or group settings. Adherence-support counseling can cover a range of behavioral or structural barriers to patients' adherence, often guiding patients to identify these barriers and collaborating with patients on strategies to overcome them. ${ }^{230}$ While provider counseling may not be able to overcome certain structural barriers, ${ }^{231}$ appropriate counseling following detection of high viral loads has been shown in some settings (though not always $)^{232}$ to improve treatment adherence and lead to re-suppression. ${ }^{214,234}$ However, lay counselors commonly receive insufficient training and support, undermining 
their ability to counsel effectively ${ }^{233}$ and highlighting an opportunity for behaviorally informed interventions.

\section{Interventions focused on this behavior}

There is particularly little research on interventions to support providers in the performance of effective counseling or to ensure that it is offered to all patients who might benefit.

MSF recommends training for providers on adherence support ${ }^{222}$ and has created training and implementation tools, including job aids to support counseling. ${ }^{230}$ The WHO also recommends that providers offering adherence support receive training on effective counseling and an algorithm to guide adherence sessions before switching patients to second-line ART. ${ }^{219}$ Neither set of recommendations has been rigorously evaluated.

Job aids and patient-facing tools may be helpful to guide conversation and support compliance with a counseling protocol. A study in Thailand provided lay counselors with a poster to help guide patients through interpretation of test results and a simple protocol to structure the conversation. Counselors quickly understood and were able to implement the protocol, and it led to improvements in patient adherence (though the study did not compare this counseling protocol with any other approach). ${ }^{234}$

\section{Potential application of behavioral economics approaches}

To provide effective counseling, providers must first determine that patients require adherence support. Behavioral economics could enhance providers' willingness to refer patients to adherence by first helping them correctly interpret patients' reasons for non-adherence. Once a need for counseling has been established, the counseling itself could then further be shaped to address the critical factors driving patients' non-adherence. Evidence that soft skills and patients' perceptions of being known and understood by providers are effective in increasing adherence to $\mathrm{ART}^{235}$ indicates that behavioral economics approaches hold promise to support these skills and improve counseling approaches.

\section{Conclusion}

Behavioral economics approaches have potential to increase the effectiveness of ART adherence counseling when providers are trained to counsel and patients do not experience structural barriers in access to care.

\subsection{Family planning}

\section{Provider counsels sexually active adolescents (15-19) on modern contraceptive use}

\section{Potential link between provider behavior and health outcomes}

Access to youth-friendly services has been shown to impact the sexual and reproductive health (SRH) and well-being of adolescents, including reducing pregnancy rates, increasing condom use, increasing knowledge of access to different contraceptive methods, and increasing use of family planning and reproductive health services. ${ }^{236,237}$ A recent evidence review found that youth-friendly services and comprehensive sexuality education were two approaches with strong evidence of positive impact on adolescent use of contraceptives. ${ }^{238}$ While school-based models may provide the most effective channel for comprehensive sexuality education, quality counseling through health providers may be a complementary or alternative channel. Particularly effective dimensions of youth-friendly services include trained, friendly, non-judgmental providers and communication and outreach to inform youth and encourage service usages. ${ }^{238}$ This review suggests the importance of provider behavior, especially given the role that bias may play, while at the same time highlighting the need for complementary approaches to effectively tackle the complex issue of adolescent contraceptive use.

\section{Interventions focused on this behavior}

One approach to changing provider behavior as it relates to adolescent family planning counseling in many settings is training. ${ }^{239} \mathrm{~A}$ literature review on the effectiveness of initiatives to improve adolescent access to and use of SRH services in low- and middle-income countries, however, found limited efficacy of providing adolescent friendliness training for providers. ${ }^{236}$ Evidence is much stronger when a combination of patient-facing and structural interventions are implemented in concert with provider training.

Another program implemented in Mozambique and Tanzania sought to mainstream youth-friendly SRH services in public-sector facilities through a range of interventions including training providers and staff in the full range of contraceptive methods, conducting facility data reviews and citizen report cards to improve performance feedback, providing job aids to CHWs, and implementing a mentorship program in addition 
to structural improvements to facilities. While not rigorously evaluated, results suggest these programs may have increased the number of new adolescent contraceptive users and use of services across time, led to increased uptake of long-acting reversible contraception (LARC), and increased correspondence between the methods clients were referred for and the methods they received at the facility, which authors used as a proxy for reduced provider bias. ${ }^{237}$

\section{Potential application of behavioral economics approaches}

Providers must decide to offer counseling and then provide it; these decisions and actions are closely tied. Behavioral economics approaches could be used to cue the provider to give counseling, including in cases where bias or mental models may inhibit this service currently. Behavioral economics could also inform counseling content, for example in order to frame messages in a way most likely to resonate with adolescents and to support intention formation.

\section{Conclusion}

Behavioral-economics-informed provider-side approaches could hold promise to increase adolescent contraceptive uptake when 1) provider bias is a key main barrier prohibiting uptake or 2) other components of youth-friendly services are being implemented.

\section{Provider counsels parents to discuss modern contraceptive use with adolescent children}

\section{Potential link between provider behavior and health outcomes}

Parental involvement in adolescent children's sexual health can help ease and accelerate the use of modern contraception; in some contexts, it is crucial to addressing adolescents' sexual health needs. ${ }^{240}$ Studies show that some providers feel uncomfortable giving contraceptives to adolescents without parental permission and fear parental repercussions. This may be particularly true in small communities where providers may be more recognized or accountable. ${ }^{241}$ There is little evidence, however, on whether providers counseling parents on discussing contraceptive use with their children has an impact on adolescent sexual health. Furthermore, the link between parent-child communication and contraceptive use is also unclear, according to the conclusions of a review on sexuality and HIV/AIDS in sub-Saharan Africa. ${ }^{242}$ The health impact of providers counseling parents on adolescent contraceptive use is therefore unclear.

\section{Interventions focused on this behavior}

Some work has been done around the relationship between parents and adolescents aimed at increasing community support for adolescent contraceptive use; however, these programs do not single out the role providers play in such efforts. ${ }^{243}$ Provider-facing interventions have targeted provider behavior when dealing with adolescents rather than attempting to address provider counseling with parents, which seems to be an unexplored area.

\section{Potential application of behavioral economics approaches}

Both providers and patients undertake decisions and actions related to this behavior. Behavioral economics approaches could be used to cue providers to discuss contraceptive use with parents-perhaps necessary, since parents most likely visit the health facility for other reasons that the provider may be focused on. Behavioral economics approaches could also be used to facilitate the conversation, for both the provider and the parent, by adjusting processes in a standard consultation so that this counseling is perceived as the default step. Another way behavioral economics could be used to facilitate the conversation would be in supporting the framing of the counseling using insights from the behavioral sciences. These insights could also be used to frame counseling takeaways best positioned to drive action on the part of the parent-in this case, to speak with their adolescent child about contraceptive use. However, because the link between this provider behavior and ultimate action on the part of the adolescent is distal, behavioral economics approaches focused on providers may hold limited promise. For instance, even after forming an intention, parents would still need to act on that intention to speak with children instead of changing their minds, deferring the conversation, or falling prey to a number of barriers that would be challenging for a provider to address after the initial counseling.

\section{Conclusion}

While behavioral economics could increase execution of this provider behavior, the evidence does not suggest that this behavior itself will have an important effect on outcomes. 


\section{Provider counsels women in unions on spacing}

\section{Potential link between provider behavior and health outcomes}

Effective counseling on spacing can prompt women to consider whether and when they would like to have a child or another child. Doing so may instill a moment of choice and help form an intention to avoid pregnancy that then facilitates family planning acceptance. Evidence that higher quality of care helps women be more decisive in pregnancy intentions and reduces the likelihood of unintended pregnancy suggests provider behavior can influence health outcomes. ${ }^{244}$

\section{Interventions focused on this behavior}

Multiple organizations have developed job aids that include this behavior as a step within larger processes. Most of these tools have been deemed effective at improving quality of care, which could affect spacing. For example, the WHO developed the Decision-Making Tool for Family Planning Clients and Providers as a job aid that providers can use to guide clients through an informed decision-making process, including discussing spacing. The tool, designed as a flipchart, was deemed useful by providers as a job aid and decision aid in Mexico ${ }^{246}$ and increased client involvement in decision-making in Nicaragua. ${ }^{147}$ Changes in contraceptive use were not measured. Population Council's Balanced Counseling Strategy: A Toolkit for Family Planning Service Providers ${ }^{c}$ is a set of job aids for counseling clients about family planning, including an 11-step algorithm, which gives providers step-by-step guidance on how to conduct a counseling session (organized according to stages of the consultation) to guide the provider through the counseling process, a set of counseling cards for contraceptive methods, and brochures for each method. Healthy timing and spacing of pregnancies is one of the topics covered in the tool. The Balanced Counseling Strategy has been shown to improve the quality of care in family planning. ${ }^{248}$ However, an evaluation of a similar tool suggests that simply providing a tool may not alone improve the quality of counseling. ${ }^{249}$

\footnotetext{
'The initial version of this toolkit has been updated and expanded. The most recent version of the toolkit, The Balanced Counseling Strategy Plus: A Toolkit for Family Planning Service Providers Working in High HIV/STI Prevalence Settings (Third Edition) is available at: https://www. popcouncil.org/research/the-balanced-counseling-strategy-plus-a-toolkit-for-family-planning-service
}

Similarly, an evaluation of the Consult and Choose program in Jordan, which included a counseling protocol that prompted providers to counsel about birth spacing and six other family-planning-oriented tasks, found that providers were less likely to discuss birth spacing than five of the other tasks. In the same program, some clients viewed a film that included messages about birth spacing. Eighty-five percent of those who watched the film reported that it was influential, and one-third of those women said they would plan their families as a result. ${ }^{250}$ Spacing and contraceptive use outcomes were not measured.

Another study involved providers discussing a narrative around postpartum pregnancy risk and distributing leaflets during typical community health activities. It found reported shifts in perceived pregnancy risk, benefits of spacing, and social support for postpartum family planning. While more than half of women interviewed stated they intended to use a modern contraceptive, only approximately one-third of women were using a modern contraceptive method. ${ }^{251}$

In Zambia, a standards-based management and recognition program focused on PMTCT led to a $41 \%$ increase in family planning counseling on spacing and contraceptive methods among HIV-positive women. The program included detailed performance standards combined with training, supportive supervision, and on-site assistance for providers. ${ }^{247}$ The evaluation did not disentangle which of these components was most effective in increasing family planning counseling on spacing specifically.

Beyond job aids, a CHW-based program in Bangladesh increased contraceptive use and significantly lowered pregnancy 24 months after a live birth through training and communication materials around spacing. ${ }^{252} \mathrm{CHWS}$ in the intervention arm received additional training on healthy spacing, postpartum family planning, and contraceptive methods and logistics management. Notably, the original study design was adapted so CHWs could distribute pills and condoms during their routine visits because participants requested contraceptives from CHWs, suggesting some intention formation occurred among participants. ${ }^{253}$ Therefore, the study's results represent the combined effect of the training, communication materials, and direct provision of family planning methods. 


\section{Potential application of behavioral economics approaches}

While the patient herself (in concert with her partner and family) must determine her desired birth spacing, a provider who counsels on ideal spacing can provide an initial moment of choice for the woman or couple to consider spacing and potentially instill an intention to avoid pregnancy by increasing her perception of pregnancy risk. Behavioral economics approaches could motivate a provider to initiate such a conversation and could provide framing more likely to be effective with a woman on reasons for spacing and contraceptive options, as evidence suggests that provider biases around women's marital status and current family size may affect the quality of counselling. ${ }^{250,254}$ The patient would then need to decide whether to use contraception and accept a method, perhaps even concurrently. If a woman did not accept a method immediately, a provider could still create a plan or appointment to provide the patient with a contraceptive method. These conversations could be especially effective when women have reasons to return to a health facility after an initial conversation, for example for PNC or children's immunizations.

\section{Conclusion}

Behavioral economics holds promise in increasing the frequency and quality of provider counseling on spacing.

\section{Provider counsels women on communicating with partner about family planning}

\section{Potential link between provider behavior and health outcomes}

Male partner involvement in family planning can help ease and accelerate the use of contraception, particularly in cultures where men are the primary decision-makers in the household. ${ }^{255}$ One avenue to increasing men's involvement is to encourage partner communication such that men are included in learning and making decisions about contraception. Providers may contribute to this dynamic in different ways, whether by counseling women on communicating with their partners about family planning or by engaging men in couples counseling. Despite this potential, there is limited evidence on the effectiveness of providers engaging women to discuss with their partners as tied to family planning uptake, and a recent evidence review on couples counseling noted both the need for more evidence as well as the great variation in approaches and definitions of couples counseling. ${ }^{256}$ One study in Jordan, for instance, found no significant difference in outcomes between counseling women alone or counseling couples together. ${ }^{257}$ Furthermore, most research focuses on efforts that engage men or couples directly (i.e., patient-facing interventions) rather than efforts that examine provider behavior as a driver for male engagement. Therefore, it is difficult to establish a definitive link between this provider behavior and family planning outcomes.

\section{Interventions focused on this behavior}

While there is an ever-growing body of interventions that seek to increase male engagement, there is a dearth of literature on interventions to influence provider behavior as it relates to encouraging male engagement or couples counseling. Engaging partners is often mentioned in counseling job aids and other tools, but we did not identify explicit interventions that seek to affect how providers frame these messages or increase couple communication. Additionally, while a recent evidence review highlighted literature on couples counseling interventions, it noted the lack of practical guidance on integrating it with other services, which could include changing provider behavior to provide this service or to conduct couples counseling more effectively. ${ }^{256}$

\section{Potential application of behavioral economics approaches}

The decisions made and actions taken to facilitate men's involvement in family planning are complex and may only tangentially involve a provider. A provider would need to determine to counsel a woman about communicating with her partner or counsel a couple together, and then do so. Behavioral economics approaches could motivate the provider to counsel, for example by addressing bias about what may be effective or by reducing discomfort around the conversation. Behavioral economics approaches might also be used to improve the quality of counseling, for example by framing messages to motivate women to discuss with their partners. However, women who were counseled alone would then need to decide to speak with their partners and follow through on this intention instead of deferring this action or changing their minds, resulting in potential for drop-off on the patient side. Couples counseling could provide a more promising avenue for a provider to increase family planning uptake, and behavioral economics could be 
used to optimize this counseling in cases where men have contact with providers.

\section{Conclusion}

havioral economics approaches could help improve provider behavior to give effective counseling to engage men when men frequently attend family planning counseling or have contact with providers at other times, though it is unclear whether this would affect family planning uptake.

\section{Provider counsels women on a full suite of methods}

\section{Potential link between provider behavior and health outcomes}

Myths and misconceptions about contraception and a lack of awareness about the available methods are often identified as barriers to contraception uptake. ${ }^{258}$ Thus, providers can play a role in improving uptake of methods by providing accurate information, answering questions, and guiding patients to select the best methods for them. Quality family planning counseling enables providers to play that role and has been shown to increase uptake of contraceptive methods. 244,250,257,259 While structural barriers such as stock-outs and scarcity of time may affect a provider's ability to counsel women on a full suite of methods, evidence also suggests that behavioral approaches can address other critical barriers that hinder optimal counseling. ${ }^{260}$ For instance, while provider counseling can play an important, positive role in helping women choose the best method for them, provider bias and assumptions may limit counseling on methods. For example, some providers may believe LARCs are only appropriate for multiparous women. ${ }^{250,261}$ Providers may also choose to counsel women on methods that are simplest for them to provide or methods that require minimal effort from the patient due to bias regarding her capacity for proper use. Focusing on provider behavior to ensure that women are counseled on a full suite of methods therefore seems to hold promise to increase family planning uptake given the importance of quality counseling.

\section{Interventions focused on this behavior}

Several job aids have been developed and implemented to encourage counseling on a full suite of methods. The WHO's Decision-Making Tool for Family Planning Clients and Providers is one such job aid. The tool is a 2-sided flipchart, with one side designed as a decision aid to help clients choose a family planning method and the other side a job aid for providers with technical information, counseling tips, and other guidance. Evaluations in Mexico and Nicaragua found the tool to be useful as both a job aid and a decision aid. ${ }^{246,247}$ Evidence from Nicaragua suggests that such tools can improve providers' counseling on contraceptive methods. Using this tool, providers worked harder to identify new clients' needs, involve them in the decision-making process, and educate them on their chosen methods more thoroughly.,247 In Tanzania, mobile job aids supported providers discussing the full suite of methods. While client and provider reports suggest that the tool improved quality of counseling, only 1 in 10 clients reported discussing the full suite of methods with the provider. ${ }^{262}$

Financial incentives for providers tied to indicators around interpersonal communication content have been used in some settings to improve provider counseling behavior, including counseling on family planning methods. In Egypt, an incentive scheme significantly improved the likelihood that providers helped patients choose a contraceptive method, though whether or not providers counseled on a full suite of methods was not necessarily measured, as the intervention sought to improve quality of counseling rather than method uptake in order to protect patient choice. $^{263}$

In Indonesia, providers who completed self-assessment exercises after a training on client-centered counseling improved the quality of their family planning counseling (as compared with providers who only attended training). The self-assessment consisted of a weekly 15- to 20-minute written exercise to reflect on a single client interaction. As a result of this intervention, providers offered more information in longer sessions; the frequency of providers' facilitative communication increased; and clients' questions and satisfaction improved. Providers who attended a peer-review group meeting in addition to completing self-assessment showed further improvements in counseling behaviors but no additional increases in client satisfaction. ${ }^{264}$
'Other existing tools include: Population Council's The Balanced Counseling Strategy Plus: A Toolkit for Family Planning Service Providers Working in High HIV/STI Prevalence Settings, Johns Hopkins School of Public Health's GATHER guide to family planning counseling; and UK's National Institute for Health and Care Excellence's guidance for LARCS. 


\section{Potential application of behavioral economics approaches}

The decision to explain the full suite of methods and providers' follow-through on this decision are nearly simultaneous and likely occur within the same clinical interaction. This close timing makes the application of behavioral-economics-informed cues more likely to affect both the decision and action. Evidence shows that knowledge about a method does not affect whether a provider counsels on that method if the provider already holds assumptions about which methods are appropriate for certain woman. ${ }^{265}$ Behavioral economics approaches could be used to help frame the counseling so that it is less susceptible to provider bias and to minimize hassles for the provider in explaining different methods. Behavioral economics could also shape counseling that effectively expands the choice set of a woman rather than being a rote exercise.

\section{Conclusion}

Behavioral economics holds promise in improving provider counseling on the full suite of methods to improve health outcomes when frequent stock-outs of those methods is not an issue.

\section{Provider counsels on condom use}

\section{Potential link between provider behavior and health outcomes}

The link between condom use and protection against pregnancy and sexually transmitted infections (STIS) as these relate to health outcomes is well-established. For providers to play a meaningful role in increasing condom use, provider counseling must address patient-side behavioral barriers that hinder condom use in the relevant population. ${ }^{266}$ For example, for adolescents in South Africa, six factors were found to hinder condom use: lack of perceived risk, peer norms, condom availability, adult attitudes toward condoms and sex, gendered power relations, and the economic context of adolescent sexuality. The evidence on the effectiveness of condom counseling on condom usage to prevent pregnancy, STIs, or HIV, however, is not strong. ${ }^{267}$ That said, there are a number of factors that make the link between this behavior and relevant health outcomes challenging to assess. For one, it is difficult to accurately measure condom use, which often relies on self-reported data prone to social desirability bias. ${ }^{268}$ Additionally, the effectiveness of counseling on condom use may be different depending on the reason for condom promotion, the target population, and the focus of the counseling session. For instance, a focus on negotiation skills rather than the benefits of condom usage may be more effective, especially among at-risk groups. ${ }^{269} \mathrm{~A}$ meta-analysis found no significant effect of counseling on condom use between study groups for HIV or pregnancy, but did find some effects for other STIs. ${ }^{270}$ Other research suggests that improving the quality of counseling could further reduce condom failure rates. ${ }^{271}$ This body of research therefore implies that while the current evidence may not strongly support the link between this provider behavior and health outcomes generally, it may be effective for certain health conditions or populations. This highlights the need for additional research to fully understand the impact of this provider behavior on outcomes.

\section{Interventions focused on this behavior}

Previous work on condom-use counseling has focused on implementing and evaluating different counseling techniques (e.g., motivational interviewing vs. general health counseling) and their impact on condom use-not necessarily the provider behavior itself or adherence to best practices. One intervention type that has been used to influence provider condom counseling is job aids. A common job aid is The A Toolkit for Family Planning Service Providers for family planning counseling in areas with high rates of HIV and STIs. ${ }^{e, 248}$ This toolkit includes an algorithm that summarizes the process for the provider, counseling cards, and brochures. While this toolkit is widely available, there does not appear to be research evaluating its effectiveness for provider behavior specifically or the link between its application and condom usage. Other job aids to support counseling on dual protection, such as PATH's counseling job aid, have been developed for use by CHWs and peer educators. ${ }^{272}$ These job aids include checklists for providers to prepare for their outreach visit or deliver counseling as well as messaging guides to address client objections. It does not appear that research on the effectiveness of these aids has been conducted.

Trainings are another common intervention that have been used to change provider behavior around counseling on condoms. Many different resources to facilitate this training exist, including K4Health's Condom Use

\footnotetext{
eThe initial version of this toolkit has been updated and expanded. The most recent version of the toolkit, The Balanced Counseling Strategy Plus: A Toolkit for Family Planning Service Providers Working in High HIV/STI Prevalence Settings (Third Edition) is available at: https://www. popcouncil.org/research/the-balanced-counseling-strategy-plus-a-toolkit-for-family-planning-service
} 
Toolkit. The impact of these trainings on provider behavior does not seem to have been researched rigorously.

\section{Potential application of behavioral economics approaches}

While a provider may counsel patients on condom use, the decision and action to use condoms must be undertaken by the patient, repeatedly and consistently. That said, provider counseling may be effective in helping form an intention or overcoming behavioral barriers on initial use by patients, for example by instructing on proper use or addressing perceived norms around condom use. Behavioral economics could be used to cue a provider to counsel on condom use and choose the right framing to facilitate the patient's engagement with counseling. Where $\mathrm{CHWs}$ are trained in condom promotion, behavioral economics approaches could help support their work with patients to overcome barriers to initiation and to support consistent use.

\section{Conclusion}

Behavioral economics appears to hold some promise as to ensuring and enhancing providers' counseling on condom use to improve outcomes when user fees for condoms are not a barrier for patients and when

1) $\mathrm{CHWs}$ are trained in condom promotion and effective in encouraging consistent use or 2) existing patient-facing interventions are effective in encouraging consistent use upon initial provider counseling.

\subsection{Provider behaviors with greatest behavioral economics potential}

The preceding summaries described the connections between provider behaviors and ultimate health outcomes, characterized existing evidence on provider-oriented interventions addressing the behavior, and assessed the potential for solutions informed by behavioral economics. The following 11 behaviors were determined to hold great promise for enhancement with behavioral economics approaches (Table 2).

TABLE 2. PROVIDER BEHAVIORS WITH GREATEST BEHAVIORAL ECONOMICS POTENTIAL

\begin{tabular}{|c|c|c|c|c|}
\hline FAMILY PLANNING & $\begin{array}{l}\text { HIV/AIDS TESTING } \\
\text { AND TREATMENT } \\
\text { ADHERENCE }\end{array}$ & $\begin{array}{l}\text { NUTRITION IN } \\
\text { CHILDREN UNDER } 2 \\
\text { YEARS OLD }\end{array}$ & $\begin{array}{l}\text { MATERNAL, } \\
\text { NEWBORN, AND } \\
\text { CHILD HEALTH }\end{array}$ & MALARIA \\
\hline $\begin{array}{l}\text { Provider counsels on } \\
\text { a full suite of methods }\end{array}$ & $\begin{array}{l}\text { Provider initiates test- } \\
\text { ing and counseling in a } \\
\text { health facility }\end{array}$ & $\begin{array}{l}\text { Provider encourages } \\
\text { breastfeeding within } 1 \\
\text { hour after delivery }\end{array}$ & $\begin{array}{l}\text { Provider follows best } \\
\text { practices during } \\
\text { facility-based delivery }\end{array}$ & $\begin{array}{l}\text { Provider conducts } \\
\text { microscopy or rapid } \\
\text { diagnostic test }\end{array}$ \\
\hline \multirow[t]{3}{*}{$\begin{array}{l}\text { Provider counsels } \\
\text { women in unions on } \\
\text { spacing }\end{array}$} & $\begin{array}{l}\text { Provider delivers } \\
\text { community-based test- } \\
\text { ing services according } \\
\text { to best practices }\end{array}$ & & $\begin{array}{l}\text { Provider gives es- } \\
\text { sential newborn care } \\
\text { immediately after } \\
\text { facility-based birth }\end{array}$ & $\begin{array}{l}\text { Provider prescribes } \\
\text { quality-assured ACT } \\
\text { with correct dosage for } \\
\text { confirmed malaria cases }\end{array}$ \\
\hline & $\begin{array}{l}\text { Provider performs vol- } \\
\text { untary assisted partner } \\
\text { notification services }\end{array}$ & & & \\
\hline & $\begin{array}{l}\text { Provider initiates } \\
\text { appropriate HIV care } \\
\text { immediately after a } \\
\text { diagnosis is confirmed }\end{array}$ & & & \\
\hline
\end{tabular}




\section{Cross-Cutting Insights and Applications}

While all individual health behaviors have unique dimensions that merit independent assessment, many behaviors in the health field are interlinked. These linkages provide opportunities for impacting multiple behaviors or identifying places where lessons can be leveraged to influence programming on different behaviors. On the programming or service-delivery side, integrated services are a clear, long-standing example of supporting patients and/or providers to overcome barriers related to one health-seeking behavior by integrating its delivery with another service for which there is already a clear touchpoint. In the behavior-change field, research on gateway behaviors presents another model for understanding the interconnectedness of behavior. ${ }^{273}$

Provider behavior change is particularly ripe for generating cross-cutting applications, as individual health providers typically offer a wide range of services and do not always conceive of their role from the narrow lens of individual health areas. The Health Communication Capacity Collaborative began through a 2016 review $^{274}$ to unpack some of the cross-cutting applications of relevance to provider behavior and specifically to identify cultural, attitudinal, and motivational factors that may impede provider behavior. We build on this analysis with a more granular examination of the common underlying behavioral drivers (contextual and psychological) at the root of these issues, which informs our discussion of where and how they may arise elsewhere. We also build on the Integrated Gateway Model for health behavior ${ }^{273}$ by unpacking the contextual features that influence providers' behavior at gateway moments and identifying behavioral insights that help explain why a patient's experience in a particular gateway moment may be uniquely powerful.

This section explores the cross-cutting insights and applications that may arise across provider behaviors, focusing on the 27 provider behaviors discussed in this report and considering three different angles (Figure 1): common behavioral barriers, transversal themes, and spillover effects. These cross-cutting insights become particularly valuable in the shift from insights about provider behavior toward solutions that drive particular desired behaviors.

- Behavioral barriers hold health care providers back from acting as they should (and in some cases, as they intend to) in their roles of providing information, guidance, and clinical services to patients. These

\section{FIGURE 1: BARRIERS, TRANSVERSAL THEMES, AND SPILLOVER EFFECTS}
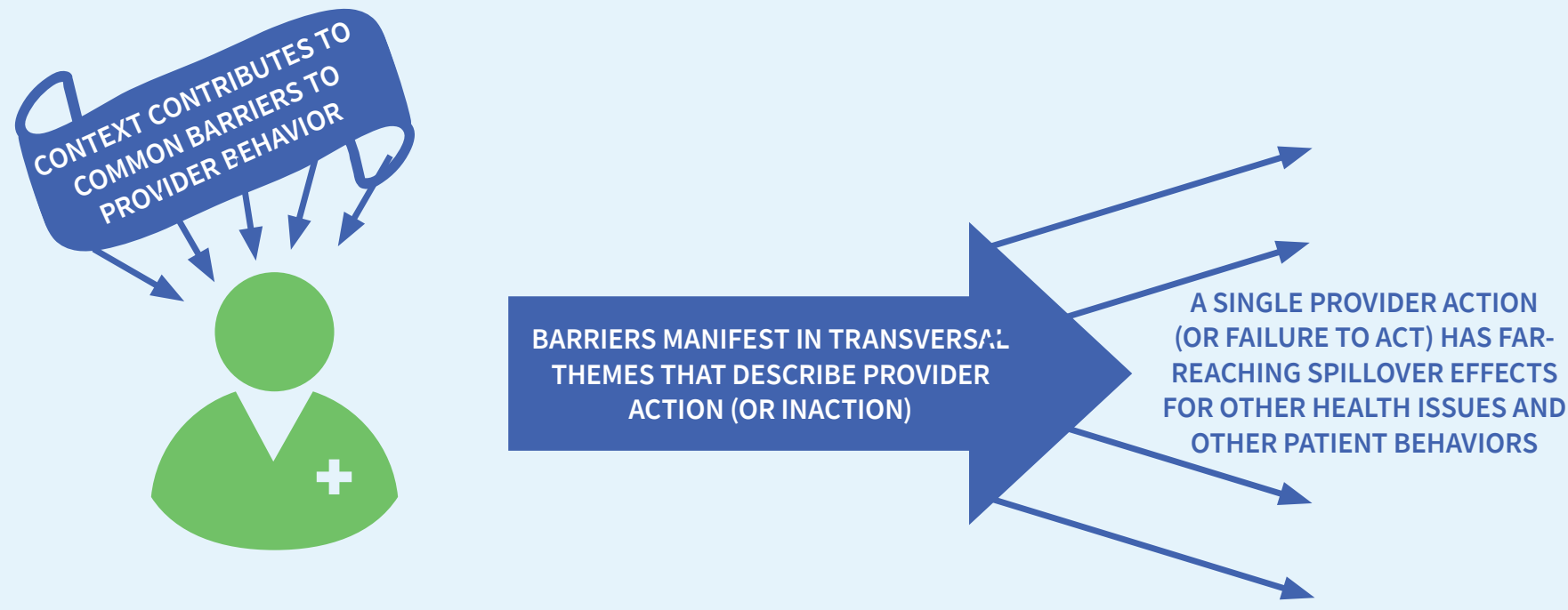
barriers arise from features of the context in which providers live and work that trigger universal psychological tendencies. Very similar barriers may appear across quite different provider behaviors, triggered by similar contextual features. Understanding how cues in one context contribute to a behavioral barrier for a single provider behavior can generate insights into how a similar barrier might arise elsewhere for a different provider or a different behavior. An example of a behavioral barrier could be status quo bias, which is a psychological tendency to resist change.

- Transversal themes are the common manifestations of various barriers in the way providers act. An understanding of when and how transversal themes result from behavioral barriers opens the door to applying components of one solution to another health issue or behavior. An example of a transversal theme is provider motivation, which may be the result of different barriers working together.

- Spillover effects include insights on how increasing uptake of a health service may increase the likelihood of future interactions with the health system in a way that could affect other health outcomes by directly or indirectly surmounting patient-side barriers through provider behavior change. A single provider behavior often has far-reaching effects on patient behaviors that it does not directly touch. Understanding the spillover effects of provider behaviors allows us to focus our efforts on the most impactful behaviors (and interventions) and eventually to move toward more comprehensive solutions that support provider behavior more broadly.

This section reviews each of the three cross-cutting dimensions in turn and ends with an analysis of which provider behaviors may show most promise to generate cross-cutting insights.

\section{Common hypothesized barriers}

Behavioral barriers arise from features in a person's context that cue certain biases or psychological tendencies. The result is a decision or action (or failure to decide or act) that is not optimal and cannot be fully explained by structural factors and rational economic decision-making. Because behavioral barriers are rooted in context, they are often unique and context specific. However, in many cases similar barriers tend to arise across types of behaviors from features that are common to many contexts. This section describes 10 such barriers, which we hypothesize to be present throughout the provider behaviors described in this report. The list below is intended to be illustrative rather than exhaustive; there are almost certainly many other barriers that commonly influence providers' actions.

Health care workers in the developing world often work in a context of profound scarcity: Supplies and resources are limited, and so is providers' time. The context of scarcity taxes mental bandwidth-attention, cognitive processing, and self-control-in ways that hinder executive functioning. ${ }^{275}$ Scarcity is a crucial feature of the context that exacerbates a number of the barriers discussed below, making them particularly likely to arise (and to have profound effects on providers' behavior) in low-resource settings.

One byproduct of this scarcity is that health care workers' attention is often stretched in many different directions. A single nurse working in a rural health facility, for example, may at one time be tasked with monitoring the condition of a woman who recently gave birth, vaccinating children, and managing patient intake-all while worrying about and fielding questions from the long line of patients waiting for care. We all have limited attentional capacity-much more limited than we tend to think. ${ }^{276-278}$ The result is that we tend to miss details without realizing it and to notice only what we expect to see. It's very difficult for a new or unexpected detail to push through the "noise," even when it might seem obvious or impossible to miss. Limited attention can lead providers to forget to perform a task or to miss details that we (and they) think they should notice. It is most likely to create barriers when the provider is juggling several tasks and where there are no salient cues to consider that task. For example, a provider may have been trained to counsel an infant's caregivers on warning signs of common infections, recognize this task as important, and still fail to do so because in the hectic moments of caring for a young child and imparting a wide array of information, they simply forget. Another provider, distracted by the long line of patients waiting for her, may fail to impart important information when counseling an HIV-positive patient on ART or may not dedicate sufficient time to convincing the patient of the importance of beginning treatment immediately.

A related consequence of scarcity is the tendency to tunnel attention and effort on what is immediately in front of us. For health care providers, this "tunnel" often 
encompasses the immediate patient or task at hand. A provider who sees a pregnant woman for her first ANC visit may tunnel on the clinical advice he is giving the woman and fail to counsel her on when to return for future visits and on the importance of doing so-an important and related piece of information that falls just outside the "tunnel" of his immediate responsibilities to monitor the woman's and child's current conditions and provide advice on any pressing clinical needs. Tunneling can also cause providers to focus on the patients who are physically present in the facility at the expense of those who are not, such as a patient who has not come to the clinic for HIV monitoring when they should have.

Busy providers are continually forced to make judgements about what activities are most important and about how to allocate their limited time and effort. In doing so, they face many occasions to assess what drives their patients to act or not act in a particular way, often with limited information about what actually influences patient behavior. These assessments are shaped by various biases and may in turn cause providers to underestimate the role that certain actions such as counseling, informing, or encouraging can play in shaping patients' behavior, with the result that they dedicate less time and effort to those tasks or skip them altogether. One such bias is the tendency to explain and predict others' behavior by their intrinsic personality traits (such as laziness or lack of interest) rather than by contextual features (such as the information or advice they've received), called the fundamental attribution error. ${ }^{279}$ When providers do not receive salient or clear feedback on the effects of providing counseling or information, it is particularly easy to develop and maintain flawed perceptions of what drives patient behavior. For example, when choosing whether to counsel a new mother on age-appropriate meals, the provider may receive little or no feedback on whether the mother follows through on the guidance. As a result, the provider may find it easy to presume that counseling has no effect and that how the mother feeds her child depends entirely on "the sort of mother she is." For other types of counseling, the cases in which providers fail to produce the desired behavior in the patient may be recalled with more ease than successful attempts-for example, a provider might hesitate to encourage an HIVpositive patient to speak with his partner if the provider can recall multiple instances when she failed to convince a patient to disclose, even if the counseling has been effective many more times.
Conversely, other contextual cues can generate overconfidence about a provider's effectiveness or understanding of the situation. We all have a tendency to be over-optimistic about our own chances of experiencing positive outcomes, in part because we focus on factors that improve our own chances while failing to recognize the degree to which others also share those factors. ${ }^{280}$ For a health care provider whose context cues him to focus on the ways in which he is in control, it may be particularly easy to overestimate the degree of that control-for example, overestimating one's ability to assess a patient's condition without a diagnostic test.

When we face the possibility of receiving or acknowledging unpleasant information, we tend to ostrich, or avoid confronting the bad news, even when doing so would be useful. ${ }^{281} \mathrm{~A}$ health care provider might avoid broaching the topic of notifying partners of HIV status, for example, if doing so requires him to discuss the patient's sexual history in a way that violates social norms or is uncomfortable. Similarly, a provider might avoid counseling a patient on a topic if she expects a negative or defensive response. Ostriching can occur even when a provider is not fully aware of the reasons he avoids certain behaviors or conversations, or doesn't recognize the extent to which the prospect of an unpleasant response is shaping her actions.

Status quo bias is a psychological tendency to resist change, which can make it painful for us to adapt to new processes, rules, or expectations. ${ }^{282}$ It can be particularly strong when switching entails a cost (even if the cost is trivial) or when it requires the actor to make some express or implied admission that the status quo is not optimal. Uncertainty about some aspect of the change can also increase our preference for what we're doing now, as the potential risks of changing loom larger in our minds than what we may stand to gain. For health care workers, status quo bias can trigger resistance to using new technologies, adapting to new clinical guidelines, or changing how they allocate time. A CHW might hesitate to comply with new guidelines for index case testing targeting individuals for HIV testing in part because doing so involves an implicit recognition that the way she was approaching testing before was not ideal. The same worker might resist changing her approach to testing because the potential downsides of the shift (failing to test an HIV-positive person who is not identified through the more targeted approach of index testing) loom larger the potential gains (reaching and testing more high-risk individuals). Status quo bias might also lead a provider to 
fail to implement a new protocol for essential newborn care because they are deterred by the time and effort it would take to become comfortable with this step; even if they do not disagree with the new protocol, have been adequately trained, and the additional effort is minimal, the hassle of this final step can exert a profound influence.

Mental models (or schemas) are representations we hold of how the world around us works and of our relationship to it. They are unconscious mental structures that are shaped by prior experience and guide us as we navigate decisions and actions. ${ }^{283}$ For health care providers, mental models of their own roles may shape perceptions of what is appropriate and important for them to do, while mental models of patients' needs may shape decisions about when, how, and to whom to offer particular services. Those mental models are formed through the training providers have received, what they observe others doing around them, and subtle cues in their environment that reinforce perceptions of how the health system works and the role they play within it. A clinician whose training has focused largely on delivering tests, administering procedures, and prescribing medication may find it difficult to align this mental model of his role with an expectation that he also convinces patients to follow through as needed. It may be easy for this clinician to assume, for example, that the task of convincing a mother to have her child vaccinated falls to a community volunteer and that his role is limited to administering the vaccine. Mental models of target patient populations can shape decisions about who needs certain services. For example, mental models of who is most at risk (which may not align with actual risk profiles) or who is most likely to follow through with treatment can influence whom a provider offers HIV testing to. Because these models have deep roots in providers' lived experiences, simply providing more training or information is often insufficient to correct them.

Our brains strive to reduce ambiguity and to form consistent understandings of people and situations. One result is halo effects, which cause us to interpret new information about a person or situation in a way that is consistent with other information we have received. ${ }^{284,285}$ For a health care provider, this might result in being less likely to counsel on warning signs for disease when a child appears healthy in other respects or placing less emphasis on the importance of PNC after an easy delivery.

\section{Transversal themes}

It is common for more than one behavioral barrier to be present in a single scenario. The combined influence of barriers manifests in transversal themes that describe the way providers act and give clues for how the context in which they work may influence other decisions and actions. This section highlights four such themes, though others certainly exist.

Provider bias refers to biases that manifest in the way providers interact with patients and the services they choose to provide in a particular case. It can influence who receives what services in what way and has implications for respectful and appropriate care of patients. Notably, provider bias does not always result (although it may result) ${ }^{286}$ in patients being poorly treated or denied services. The roots of provider bias are complex, stemming from numerous different cognitive biases (systematic errors in memory or evaluation) including attribution bias as well as from other types of barriers. The consequence is that health care services may not be provided optimally to all patients.

Provider bias might, for example, help explain a provider's choice to counsel some but not all HIV-positive patients on disclosing their status to their partners because they make assumptions about who is most likely to follow through or who is most at risk or because the conversation may be particularly uncomfortable with certain types of patients. It might also explain a provider's willingness to counsel adolescents on family planning or the way the provider carries out the conversation, which may be shaped by views about who "should" be having sex, the types of advice adolescents need, and the role they should play as a health care provider in supporting family planning choices among this population.

Provider motivation refers to the degree of motivation providers have to carry out their defined duties as well as to go above and beyond in service of the broader purpose of their role. It includes motivation of volunteer as well as professional cadres of health care providers, recognizing the substantial differences between the two. Motivation determines the level of effort providers are willing to make, their willingness to devote time and energy to tasks they perceive to be outside of their delineated responsibilities, and even how they think about what those responsibilities are. While provider motivation may in large part be explained by structural factors 
such as compensation, workload, and training and preparation, there may also be significant behavioral factors at play. For example, the tone and content of feedback providers receive from patients as well as supervisors (and the feedback that is most salient to them) may profoundly influence their mental models of their roles as providers. These mental models can, in turn, influence their motivation to complete certain tasks. As a result, there may be great potential for behavioral science to inform solutions to increase motivation, even when structural changes are not possible.

Provider motivation might, for example, explain a midwife's willingness to devote the time to counseling a new mother on PNC or encouraging breastfeeding, especially if she views these as tasks outside of her core duties that can or should be done by a volunteer. It might also explain a CHW's decisions about how much of their time doing outreach and testing for HIV they dedicate to the hardest-to-reach populations.

\section{A third transversal theme is providers' navigation of} referral systems and coordination and information sharing between providers within and outside a facility. This interaction is integral to carrying out many of the behaviors described in this report. Providers' willingness and ability to interact effectively with other providers may be influenced by mental models of their own and other providers' roles, by their comfort navigating referrals, and in some cases by patients' mental models of who can and should provide certain services.

Interaction and referrals are particularly important and particularly challenging in the case that coordination between facility-based providers and community volunteers is necessary. This happens whenever one portion of the continuum of care is provided in the community and the other in a facility or when community-based volunteers rely on information from the facility (or vice versa). For example, follow-up with HIV-positive patients who have stopped treatment may be carried out by a volunteer who seeks out the patient in the community based on information provided by the facility and then refers the patient back to the facility to continue care. This relies on providers in the community and facility holding consistent views of their respective roles and dedicating effort to ensuring that there is good coordination and continuity from the patient's perspective.

Uptake of new technology or clinical guidelines by providers is a final transversal theme that describes providers' willingness to adopt new resources, protocols, and guidance and use them consistently and appropriately. Status quo bias predicts a general resistance to change, but several other behavioral barriers may also help to explain the seemingly paradoxical behavior of providers when presented with new tools that can help them provide care more efficiently or effectively. Providers may react negatively to new technology or guidelines if they contradict mental models of the provider's role or of what appropriate care entails or if providers do not fully understand the implications of these new practices for patient outcomes. In other cases, a provider may procrastinate in taking the steps needed to assimilate new practices as he "tunnels" on the more immediately pressing needs of the patient in front of him.

Hesitance to take up new technology might be seen in a provider's failure to adhere to new guidelines for viral load monitoring of HIV-positive patients that she is less comfortable applying or in failure to counsel patients on the latest best practices related to infant nutrition that she does not fully understand or accept. A provider might fail to apply new guidelines for essential newborn care if she perceives some inconsistency with her prior training or if she views some of the tasks she is asked to carry out as better suited to other providers.

\section{Spillover effects}

Potential spillover effects are present in three different manifestations:

\section{Facilitating movement along the treatment continuum,} for example by helping patients to progress from HIV counseling and testing to treatment or ensuring that women who attend ANC return to the facility for birth and PNC. Solutions that address barriers arising early in the continuum or relating directly to transition between different stages of care may be particularly relevant to ensure continuity of care.

\section{Creating another touchpoint with the health system} through which providers can offer different types of health services, such as offering HIV testing to a young woman who comes to the facility for family planning or counseling a new mother on birth spacing and family planning when she brings her child for vaccinations. Creating additional touchpoints may be most valuable for patients who have limited contact with the health care system (such as youth) or where there are critical health 
care needs that patients do not always proactively seek out (such as HIV testing).

Increasing comfort or confidence to seek care for other needs or at later times by offering respectful and patient-centered care that empowers patients to access the health care system for later needs. A positive experience during and immediately after childbirth may cause a woman to more quickly seek care for a sick child months or years later; it may also increase her willingness to visit the facility for subsequent pregnancies.

\section{Behaviors with the most potential for cross-cutting lessons and impact}

There are cross-cutting aspects to each of the behaviors we examined in this review, but the potential for cross-cutting insights and applications is particularly great for certain behaviors. For each of the 27 behaviors described in Section 3, we estimated the applicability of common hypothesized barriers, transversal themes, and spillover effects. We classified the following seven behaviors as having the highest potential for cross-cutting applications: at least four common hypothesized barriers shared with multiple behaviors across health areas, at least three relevant transversal themes, and the high likelihood of at least two spillover effects. 
TABLE 3: EXAMPLES OF CROSS-CUTTING DIMENSIONS FOR THE SEVEN BEHAVIORS WITH HIGHEST POTENTIAL FOR CROSS-CUTTING INSIGHTS

\begin{tabular}{|c|c|c|c|}
\hline BEHAVIOR & $\begin{array}{l}\text { COMMON HYPOTHESIZED } \\
\text { BARRIERS }\end{array}$ & TRANSVERSAL THEMES & SPILLOVER EFFECTS \\
\hline $\begin{array}{l}\text { Provider initiates appropriate } \\
\text { HIV care immediately after a } \\
\text { diagnosis is confirmed }\end{array}$ & $\begin{array}{l}\text { Provider doesn't receive salient } \\
\text { feedback on effectiveness of } \\
\text { referrals and uses faulty heuris- } \\
\text { tics to assess her performance } \\
\text { Mental models of what appro- } \\
\text { priate treatment entails limit } \\
\text { what the provider feels she is } \\
\text { "able" to do }\end{array}$ & $\begin{array}{l}\text { Biased assumptions about who } \\
\text { is most likely to follow through } \\
\text { Motivation needed to exert the } \\
\text { effort to counsel resistant or } \\
\text { confused patients } \\
\text { Adoption of new treatment } \\
\text { guidelines or comfort treating } \\
\text { certain types of patients }\end{array}$ & $\begin{array}{l}\text { Treatment adherence } \\
\text { Testing of partners } \\
\text { Touchpoint with facility to } \\
\text { provide other care }\end{array}$ \\
\hline $\begin{array}{l}\text { Provider performs voluntary } \\
\text { assisted partner notification } \\
\text { services }\end{array}$ & $\begin{array}{l}\text { Provider avoids an uncom- } \\
\text { fortable conversation about } \\
\text { patient's sexual history } \\
\text { When attention is focused on } \\
\text { the patient at hand, provider } \\
\text { may not be cued to consider } \\
\text { testing and care of others }\end{array}$ & $\begin{array}{l}\text { Biased assumptions about } \\
\text { which method of notification is } \\
\text { most appropriate for a particu- } \\
\text { lar patient } \\
\text { Adoption of new guidelines for } \\
\text { assisted notification (rather } \\
\text { than passive referral) }\end{array}$ & $\begin{array}{l}\text { Testing and treatment of } \\
\text { partners } \\
\text { Treatment adherence for both } \\
\text { partners }\end{array}$ \\
\hline $\begin{array}{l}\text { Provider encourages four or } \\
\text { more ANC visits }\end{array}$ & $\begin{array}{l}\text { Provider's mental model of her } \\
\text { role may not include convincing } \\
\text { patients to seek care } \\
\text { When tunneling on immediate } \\
\text { clinical advice, it is easy to } \\
\text { overlook future needs }\end{array}$ & $\begin{array}{l}\text { Biased assumptions about who } \\
\text { needs ANC most or who is least } \\
\text { likely to come } \\
\text { Coordination between commu- } \\
\text { nity-based and facility-based } \\
\text { providers }\end{array}$ & $\begin{array}{l}\text { Facility-based delivery } \\
\text { Later care-seeking, including } \\
\text { for PNC, family planning, care } \\
\text { for children, and care for next } \\
\text { pregnancy }\end{array}$ \\
\hline $\begin{array}{l}\text { Provider follows best practices } \\
\text { during facility-based delivery }\end{array}$ & $\begin{array}{l}\text { Provider tunnels on needs that } \\
\text { she perceives as most urgent } \\
\text { and deprioritizes respectful } \\
\text { care } \\
\text { Steps that do not involve using } \\
\text { equipment or documentation } \\
\text { are less salient and easy to skip }\end{array}$ & $\begin{array}{l}\text { Biased views of what type of } \\
\text { care certain mothers deserve } \\
\text { Uptake of new clinical guide- } \\
\text { lines that are perceived as } \\
\text { inconsistent with training or } \\
\text { supervision }\end{array}$ & $\begin{array}{l}\text { Later care-seeking for other } \\
\text { needs, including for PNC and } \\
\text { family planning } \\
\text { Facility-based delivery for next } \\
\text { pregnancy }\end{array}$ \\
\hline $\begin{array}{l}\text { Provider gives essential new- } \\
\text { born care immediately after } \\
\text { facility-based birth }\end{array}$ & $\begin{array}{l}\text { Mental model of the function of } \\
\text { the checklist means that certain } \\
\text { steps are viewed as optional } \\
\text { After a healthy delivery, pro- } \\
\text { vider may underestimate how } \\
\text { critical newborn care is }\end{array}$ & $\begin{array}{l}\text { Motivation to perform tedious } \\
\text { tasks when there's no oversight } \\
\text { of compliance } \\
\text { Uptake of guidelines that may } \\
\text { be perceived as inconsistent } \\
\text { with practical experience }\end{array}$ & $\begin{array}{l}\text { Good practices for child } \\
\text { nutrition } \\
\text { Later care-seeking for other } \\
\text { needs }\end{array}$ \\
\hline $\begin{array}{l}\text { Provider encourages PNC } \\
\text { check-up within two days of } \\
\text { birth }\end{array}$ & $\begin{array}{l}\text { Tunneling on immediate needs } \\
\text { of the mother and baby leads } \\
\text { provider to overlook future } \\
\text { care-seeking } \\
\text { Provider may avoid broaching } \\
\text { the topic if it means confront- } \\
\text { ing the mother's resistance to } \\
\text { return }\end{array}$ & $\begin{array}{l}\text { Coordination and referrals be- } \\
\text { tween community-based and } \\
\text { facility-based providers }\end{array}$ & $\begin{array}{l}\text { Good practices for child } \\
\text { nutrition } \\
\text { Vaccinations } \\
\text { Family planning counseling } \\
\text { Touchpoint with facility to } \\
\text { provide other care }\end{array}$ \\
\hline $\begin{array}{l}\text { Provider encourages full com- } \\
\text { pletion of timely vaccinations } \\
\text { for children under two years old }\end{array}$ & $\begin{array}{l}\text { Provider doesn't receive salient } \\
\text { feedback on performance, } \\
\text { making it easy to assume that } \\
\text { performance is adequate } \\
\text { Facility-based providers may } \\
\text { have a mental model of their } \\
\text { role as only administering } \\
\text { the vaccine, not encouraging } \\
\text { follow-up }\end{array}$ & $\begin{array}{l}\text { Biased assumptions about who } \\
\text { needs vaccination most or is } \\
\text { least likely to seek it out } \\
\text { Outreach by community-based } \\
\text { workers and coordination with } \\
\text { facility about who has not } \\
\text { completed }\end{array}$ & $\begin{array}{l}\text { Family planning counseling } \\
\text { Counseling on warning signs } \\
\text { Touchpoint with facility to } \\
\text { provide other care }\end{array}$ \\
\hline
\end{tabular}


This gap analysis distills the availability and strength of evidence for provider-facing interventions among priority health areas described in Section 3. These interventions include SBC interventions but also other interventions that sought to affect provider behavior after initial clinical skills were in place. Overall, the evidence on these interventions is scant. The interventions that have been studied to date and are included in this review include some behavioral angle or application but do not necessarily use the explicit lens of behavioral economics in their design or evaluation. This more comprehensive approach to compiling interventions allows for the identification of gaps where there are opportunities to develop new interventions informed by behavioral economics and also where there may be promise to enhance existing interventions with behavioral economics approaches regardless of whether or not they were initially conceived as SBC interventions.

The wide variety of provider-facing interventions encountered in this review fall into several general categories informed by a taxonomy of intervention types: ${ }^{287}$

- Training and education interventions reinforce or supplement providers' existing knowledge. While they are common (and frequently recommended by global entities such as the WHO) and some have demonstrated efficacy, the evidence of their impact on provider behavior is mixed. Targeted, repetitive interventions with applied and interactive components where accompanying media and appropriate setting have been carefully considered appear to be most effective. ${ }^{288}$ While training as a format is not typically viewed as a behavioral economics intervention, different workshops, especially values clarification or mindset interventions, do build on behavioral science research, and training messaging could be enhanced by behavioral economics. Examples of this category cited in the review include provider training on youth-friendly services or training on ART adherence.

- Managerial approaches involve supportive supervision, mentorship, or peer support or the implementation of audits, targets, monitoring, or feedback to improve performance. These have been implemented more frequently and have shown more success than other intervention types. While these interventions have often been viewed as health systems strengthening interventions, behavioral economics often employs similar approaches with a more behavioral lens, and some of these approaches do build on behavioral science research. Examples of this category cited in the review include the clinical mentorship program to support adherence to the SCC or targets and accompanying feedback for viral load tests.

- Institutional process improvements include checklists or case sheets to enhance provider behaviors across facilities or groups, and individual process improvements target individuals' performance through microplanning devices, job aids, and targeted reminders. The limited evidence available for these types of interventions suggests that they are promising, although they have not been implemented or studied as frequently as others. Many of these interventions build on principles from behavioral economics or have been employed by behavioral economics practitioners. Examples of this category cited in the review include job aids for family planning counseling or self-administered provider guides for ICMI.

- Supplementary patient-facing materials furnish assistance to providers primarily when they counsel, explain, or otherwise convey information to patients. Few of the programs identified in our review employed these materials. Behavioral economics may employ such approaches when it is clear that patient-side factors affect provider behaviors, and this format may address patient barriers. Examples of this category cited in the review include brochures on condom usage post-counseling or leaflets on pregnancy risk to support spacing.

- Financial incentives reward providers for a desired behavior. Few of the programs identified in our review linked financial incentives with the specific provider behaviors of focus, although there is a broader body of evidence on financial incentives for health care providers showing mixed results. ${ }^{289}$ Behavioral economics may employ incentives as a means to drive behavior change. Examples of this 
category cited in the review include performance incentives to increase ANC coverage and facility-based delivery.

For each behavior, we assessed the availability and strength of evidence for existing interventions targeting the behavior. Because our literature review was not comprehensive, we assessed analytical quality according to a simple heuristic. ${ }^{f}$ Seventeen of the behaviors described in Section 3 have moderate or strong promise for behavioral economics solutions. Further research would be fruitful for all of these behaviors, but consideration of the availability and strength of existing evidence hints at where and how further research may be particularly valuable.

Where existing evidence is limited or mixed (see Table 4), research will be particularly valuable to investigate the behavioral barriers at play and to explore and test the potential of behavioral economics approaches. Where evidence is promising (see Table 5), further research could be particularly valuable to deepen understanding of the intervention types that show early promise (most have not been evaluated for persistence of positive effects). Further research in these areas could contribute to refinements of interventions from a behavioral economics angle to increase their effectiveness in improving provider behavior.
TABLE 4: INTERVENTIONS WITH LIMITED OR MIXED EVIDENCE OF IMPACT ACROSS ALL INTERVENTION TYPES THAT MAY PARTICULARLY BENEFIT FROM RESEARCH TO DEVELOP BEHAVIORAL ECONOMICS SOLUTIONS

\section{HEALTH AREA PROVIDER BEHAVIOR}

\begin{tabular}{|c|c|}
\hline FPRH & $\begin{array}{l}\text { Provider counsels women in unions on } \\
\text { spacing }\end{array}$ \\
\hline FPRH & Provider counsels on condom use \\
\hline HIV & $\begin{array}{l}\text { Provider performs voluntary assisted part- } \\
\text { ner notification services }\end{array}$ \\
\hline HIV & $\begin{array}{l}\text { Provider communicates results of HIV moni- } \\
\text { toring to patients }\end{array}$ \\
\hline $\mathrm{MNCH}$ & $\begin{array}{l}\text { Provider encourages full completion of } \\
\text { timely vaccinations for children under two } \\
\text { years old }\end{array}$ \\
\hline Malaria & Provider conducts microscopy or RDT \\
\hline Malaria & $\begin{array}{l}\text { Provider prescribes quality-assured ACT with } \\
\text { right dosage }\end{array}$ \\
\hline
\end{tabular}

fWe consider randomized control trials and observational analyses not at risk from selection bias, uncontrolled confounding and other biases as rigorous, while pre-post tests, descriptive analyses, and others do not meet this standard. 


\section{TABLE 5: INTERVENTIONS WITH PROMISING SUGGESTION OF IMPACT THAT WARRANT FURTHER EXPLORATION TO ENHANCE EFFECTIVENESS WITH BEHAVIORAL ECONOMICS}

\begin{tabular}{|c|c|c|}
\hline $\begin{array}{l}\text { HEALTH } \\
\text { AREA }\end{array}$ & PROVIDER BEHAVIOR & INTERVENTIONS WITH INITIAL PROMISE \\
\hline FPRH & $\begin{array}{l}\text { Provider counsels sexually active adoles- } \\
\text { cents on modern contraceptive use }\end{array}$ & $\begin{array}{l}\text { Managerial approaches: multi-pronged approach that includes data reviews, citizen } \\
\text { report cards, job aids, and a mentorship program }\end{array}$ \\
\hline \multirow[t]{2}{*}{ HIV } & \multirow{2}{*}{$\begin{array}{l}\text { Provider initiates testing and counseling in a } \\
\text { health facility }\end{array}$} & Institutional process improvement: supervisory checklist \\
\hline & & Financial incentives: pay-for-performance scheme related to multiple outcomes \\
\hline HIV & $\begin{array}{l}\text { Provider delivers community-based services } \\
\text { according to best practices }\end{array}$ & Managerial approaches: data monitoring system paired with individualized follow-up \\
\hline \multirow[t]{2}{*}{ HIV } & \multirow[t]{2}{*}{ Provider ensures accurate testing } & Training and education initiatives: training of lay providers \\
\hline & & $\begin{array}{l}\text { Managerial approaches: performance standards and checklists combined with } \\
\text { supportive supervision }\end{array}$ \\
\hline \multirow[t]{2}{*}{ HIV } & \multirow{2}{*}{$\begin{array}{l}\text { Provider initiates appropriate HIV care } \\
\text { immediately after a diagnosis is confirmed }\end{array}$} & Managerial approaches: educational outreach accompanied by supervision \\
\hline & & $\begin{array}{l}\text { Institutional process improvement: toolkit including a checklist and authorization } \\
\text { letters; 2-part referral forms; ART eligibility screening tool }\end{array}$ \\
\hline HIV & Provider regularly monitors viral loads & Managerial approaches: clinical mentorship programs \\
\hline HIV & Provider counsels if viral loads are not ideal & Individual process improvement: algorithm to guide adherence sessions \\
\hline \multirow[t]{2}{*}{ Nutrition } & \multirow[t]{2}{*}{$\begin{array}{l}\text { Provider encourages breastfeeding within } \\
\text { one hour after delivery }\end{array}$} & $\begin{array}{l}\text { Training and education initiatives: training on skills to support mothers during early } \\
\text { breastfeeding }\end{array}$ \\
\hline & & $\begin{array}{l}\text { Institutional process improvement: comprehensive program involving institutional } \\
\text { breastfeeding policy, training, and providing support for mothers; checklist and } \\
\text { coaching to encourage breastfeeding }\end{array}$ \\
\hline \multirow[t]{2}{*}{ Nutrition } & \multirow[t]{2}{*}{$\begin{array}{l}\text { Provider encourages exclusive breastfeeding } \\
\text { for six months }\end{array}$} & $\begin{array}{l}\text { Training and education initiatives: training on skills and communication combined } \\
\text { with messaging and community mobilization }\end{array}$ \\
\hline & & $\begin{array}{l}\text { Individual process improvement: counseling cards and technical reference guides } \\
\text { combined with training }\end{array}$ \\
\hline \multirow[t]{2}{*}{ Nutrition } & \multirow{2}{*}{$\begin{array}{l}\text { Provider counsels on minimum meal } \\
\text { frequency and nutritious, age- } \\
\text { appropriate foods }\end{array}$} & Training and education initiatives: course on complementary feeding \\
\hline & & Managerial approaches: supportive supervision following self-administered surveys \\
\hline \multirow[t]{2}{*}{$\mathrm{MNCH}$} & \multirow{2}{*}{$\begin{array}{l}\text { Provider gives relevant information to care- } \\
\text { givers on signs, symptoms, and appropriate } \\
\text { treatment of immediate risks (such as acute } \\
\text { respiratory infections and diarrheal disease) }\end{array}$} & $\begin{array}{l}\text { Managerial approaches: supervisory system (including regular reporting) and peer } \\
\text { support groups following training }\end{array}$ \\
\hline & & $\begin{array}{l}\text { Training and education initiatives: training of CHWs combined with provision of } \\
\text { medical supplies }\end{array}$ \\
\hline $\mathrm{MNCH}$ & Provider encourages four or more ANC visits & Institutional process improvement: performance teams to identify and address gaps \\
\hline \multirow[t]{3}{*}{$\mathrm{MNCH}$} & \multirow[t]{3}{*}{ Provider encourages facility-based delivery } & Managerial approaches: quarterly supervision combined with training \\
\hline & & Institutional process improvement: performance teams to identify and address gaps \\
\hline & & Individual process improvement: using birth plans with patients \\
\hline \multirow[t]{2}{*}{$\mathrm{MNCH}$} & \multirow{2}{*}{$\begin{array}{l}\text { Provider follows best practices during } \\
\text { facility-based delivery }\end{array}$} & Managerial approaches: quarterly supportive supervision combined with training \\
\hline & & $\begin{array}{l}\text { Institutional process improvement: checklist combined with coaching visits; audits } \\
\text { and feedback mechanisms; provider recognition; changes to clinical environment }\end{array}$ \\
\hline \multirow[t]{3}{*}{$\mathrm{MNCH}$} & \multirow[t]{3}{*}{$\begin{array}{l}\text { Provider gives essential newborn care im- } \\
\text { mediately after facility-based birth }\end{array}$} & $\begin{array}{l}\text { Training and education initiatives: trainings in essential newborn care and } \\
\text { resuscitation }\end{array}$ \\
\hline & & Managerial approaches: coaching combined with checklist implementation \\
\hline & & Institutional process improvement: checklist combined with coaching \\
\hline $\mathrm{MNCH}$ & $\begin{array}{l}\text { Provider encourages post-natal check-up } \\
\text { within two days of birth }\end{array}$ & $\begin{array}{l}\text { Individual process improvement: using birth plans and discussing birth prepared- } \\
\text { ness with patients }\end{array}$ \\
\hline \multirow[t]{2}{*}{ Malaria } & \multirow{2}{*}{$\begin{array}{l}\text { Provider counsels pregnant women and } \\
\text { mothers on the importance of sleeping } \\
\text { under an ITN and provides IPTp }\end{array}$} & Training and education initiatives: retraining on delivery, timing, and dosage of IPTp \\
\hline & & $\begin{array}{l}\text { Managerial approaches: supportive supervision combined with community } \\
\text { mobilization }\end{array}$ \\
\hline
\end{tabular}




\section{Conclusion: The Path Forward}

\section{Using these insights for research and impact}

Our aim is for insights from this review to be used to inform two types of critical decisions about programming and research on provider behavior change:

- Designing and adapting programs to maximize impact, adapting proven solutions to new contexts, employing lessons from the literature to address different challenges, and making strategic decisions about where and how to intervene for maximum impact on health outcomes.

- Shaping the research agenda to fill critical gaps in understanding of provider behavior, choosing research projects with an eye toward generating novel and broadly applicable insights, and considering how the results can be applied across contexts, behaviors, and health areas so that the impact of provider-facing behavioral interventions continues to grow.

\section{Considerations for future research}

Given the nascent stage of both research and practice in this field, our primary focus is on shaping the research agenda to develop a more robust evidence base. This review provides a first step toward identifying priority research areas based on five considerations:

1. Potential for behavioral economics solutions. For nearly all of the provider behaviors included in this review, there is potential for behavioral economics to impact health outcomes by increasing the likelihood that a provider performs an action or improving the way in which they do so. However, certain behaviors show particularly great potential for behavioral economics solutions because the provider behavior has a strong link to health outcomes and because there is a promising angle from which an intervention might improve provider behavior. Section 3 (Evidence for Behavioral Economics Solutions) identifies 11 such behaviors for which the promise of behavioral economics' approaches focused on providers is particularly great.

2. Limited existing research or early promise of impact. Although none of the provider behaviors or intervention types discussed in this review have been fully explored, the evidence for behavioral economics interventions is particularly sparse for certain provider behaviors. For other behaviors, the evidence suggests that there may be positive impact from behavioral economics interventions, but how and under what circumstances those interventions influence provider behavior is not yet fully understood. In both of these areas, further research will be particularly valuable to uncover new solutions. Section 5 (Gap Analysis) identifies seven such provider behaviors for which the need for additional research is particularly great.

3. Shared hypothesized barriers and transversal themes with other behaviors. Certain behaviors share a particularly large number of hypothesized behavioral barriers with other behaviors, which may manifest in similar ways for other decisions and actions in other health areas. Research that sheds light on how cues in one context hold back provider behavior is likely to be broadly relevant, as it generates insights into how similar barriers might arise elsewhere for a different provider or in another behavior. Section 4 (Cross-Cutting Insights and Applications) identifies seven such provider behaviors that are particularly likely to generate cross-cutting insights.

4. Potentially great spillover effects on other health behaviors and outcomes. Some provider behaviors are particularly likely to result in spillover effects for other health behaviors and outcomes, for example by creating additional touchpoints between individuals and the health system or by offering care at a crucial moment in a way that encourages future care-seeking behavior for other needs. Research that informs a solution to improve these particularly impactful provider behaviors is likely to have far-reaching direct and indirect consequences for health outcomes. Section 4 (Cross-Cutting Insights 
and Applications) identifies seven such provider behaviors with high potential for spillover effects.

5. The context is conducive to behavioral economics solutions. Behavioral solutions hold the most promise in certain contexts, such as those where the provider behavior is not currently being performed optimally (with consequent effects for health outcomes) and where the primary barriers are not structural. Section 3 describes potentially relevant structural considerations for each provider behavior, which should be investigated within the context in which research is conducted.

\section{Example: application to formative research in Zambia}

ideas42 used the insights generated through this review to select a focus area for formative research on provider behavior change in Zambia. This research, conducted through the Breakthrough RESEARCH project, entails using ideas42's behavioral mapping methodology combined with qualitative fieldwork to refine and validate hypotheses about the relevant behavioral barriers at play. This research will inform our design of a solution to improve provider behavior.

Our research project was scoped according to the five considerations outlined above, which led us to focus on the following behavior: Provider follows best practices during facility-based delivery (Section 3.1).

1. Potential for behavioral economics solutions. Providers' compliance with good practices during delivery has great potential to improve health outcomes for mothers and infants. Because these behaviors happen at a discrete point in time and do not rely heavily on client intermediation or specialized equipment and supplies, there are promising angles through which providers' behavior may be influenced by behavioral interventions.

2. Limited existing research or early promise of impact. Although there is evidence of positive impact on providers' compliance with good practices during delivery from certain types of interventions (such as trainings), there is limited evidence on other types of interventions. There is particularly little evidence on interventions to promote respectful care, suggesting that formative research in this area has potential to contribute meaningfully to the knowledge base.

\section{Shared hypothesized barriers and transversal} themes with other behaviors. Insights uncovered through research on this behavior are likely to be applicable to other provider behaviors across a range of health areas. For example, this research may generate broadly applicable insights into how providers perceive tradeoffs between urgent clinical needs and respectful care, the influence of provider bias on how different types of patients are treated, and how providers make (and remember) choices about new clinical guidelines.

4. Potentially great spillover effects on other health behaviors and outcomes. The experience during childbirth is likely to have a particularly strong impact on how the mother perceives the health system and quality of care at the facility she attends. A positive, respectful experience may lead her to be more likely to return for PNC and to be quicker to seek care for a sick child months or years later; it may also increase her willingness to visit the facility for subsequent deliveries.

5. The context is conducive to behavioral economics solutions. Despite the country's relatively high rates of facility-based birth (67\%), ${ }^{290}$ maternalg and neonatal ${ }^{\mathrm{e}}$ mortality in Zambia are high. Although progress has been made in reducing both in recent years, they remain substantially higher than Sustainable Development Goal targets. ${ }^{\text {h }}$ Some of the leading causes of maternal and neonatal mortality, including hemorrhage, infection, and asphyxia, are avoidable with adherence to good clinical practices during and immediately following birth. ${ }^{293}$ However, there is evidence of gaps in adherence to recommended clinical practices during routine and emergency obstetric care in Zambia. ${ }^{294,295}$ There is also evidence that providers skip tasks even when the necessary supplies and equipment are available (i.e., that structural barriers are not always relevant). ${ }^{295}$ Moreover, there is evidence that providers frequently fail to provide respectful care during facility-based delivery. ${ }^{296-298}$ This suggests that the Zambian context

'The 2015 maternal mortality rate in Zambia was 224 per 100,000 live births. ${ }^{291,292}$

hSDG target for maternal mortality is under 70 per 100,000 live births by 2030. ${ }^{291}$ SDG target for neonatal mortality is under 12 deaths per 1,000 live births by $2030 .^{292}$ 
is very appropriate for behavioral interventions to improve compliance with good clinical practices and respectful care during facility-based delivery, with consequent benefits for maternal and neonatal health.

\section{Connecting research to impact}

The aims of shaping research and maximizing impact are interconnected. As carefully scoped and designed research fills gaps in understanding of behavioral economics approaches to provider behavior change, these lessons can translate to improvements in programming.

FIGURE 2: CONNECTING RESEARCH TO IMPACT

\section{SHAPE THE RESEARCH AGENDA}

1. Explore behaviors for which there is a promising angle for behavioral economics solutions
TO

\section{MAXIMIZE IMPACT}

Match behavioral economics solutions to behavioral problems
2. Explore behaviors where there are gaps in research and/or show early promise of impact
Gain insight into the behavioral aspects of the problem
Measure the impact of new solutions on provider behavior and health outcomes
Adapt solutions with proven success

Extrapolate learnings from one provider behavior to others

Choose programmatic focus to maximize overall impact
4. Consider potential spillover effects on other health behaviors and outcomes
Measure spillover effects from improving a provider behavior
Unpack the specific barriers and themes within behaviors
Validate behavioral and structural aspects of the context
Design for both behavioral and structural barriers solutions (i.e., challenges are not primarily structural) 
1. Rice, Thomas. 2013. "The behavioral economics of health and health care," Annual Review of Public Health 34(1): 431-47. doi: 10.1146/ annurev-publhealth-031912-114353.

2. Thorgeirsson, Tryggvi, and Ichiro Kawachi. 2013. "Behavioral economics," American Journal of Preventive Medicine 44(2): 185-89. doi: 10.1016/j. amepre.2012.10.008.

3. Trujillo, A. J. et al. 2015. "Applying behavioural economics to health systems of low- and middle-income countries: what are policymakers' and practitioners' views?," Health Policy and Planning 30(6): 747-58. doi: 10.1093/heapol/czu052.

4. Mangham, L. J., and K. Hanson. 2010. "Scaling up in international health: what are the key issues?," Health Policy and Planning 25(2): 85-96. doi: 10.1093/ heapol/czp066.

5. Bhutta, Zulfiqar A, Jai K Das, Rajiv Bahl, and Joy E Lawn. 2014. "Can available interventions end preventable deaths in mothers, newborn babies, and stillbirths, and at what cost?", The Lancet 384(9940): 347-70. doi: 10.1016/S0140-6736(14)60792-3

6. Semrau, Katherine E.A. et al. 2017. "Outcomes of a coaching-based WHO Safe Childbirth Checklist Program in India," New England Journal of Medicine 377(24): 2313-24. doi: 10.1056/NEJMoa1701075.

7. Beltman, J. J. et al. 2013. "Local health workers' perceptions of substandard care in the management of obstetric hemorrhage in rural Malawi," BMC Pregnancy and Childbirth 13(39). doi: 10.1186/1471-2393-13-39.

8. Opondo, Charles et al. 2009. "Are hospitals prepared to support newborn survival? An evaluation of eight first-referral level hospitals in Kenya," Tropical Medicine \& International Health 14(10): 1165-72. doi: 10.1111/j.1365-3156.2009.02358.x.

9. Patabendige, Malitha, and Hemantha Senanayake. 2015. "Implementation of the WHO Safe Childbirth Checklist Program at a tertiary care setting in Sri Lanka: a developing country experience," BMC Pregnancy and Childbirth 15(12). doi: 10.1186/ s12884-015-0436-0.
10. Bradley, Janet, et al. 2017. "Improving the knowledge of labour and delivery nurses in India: a randomized controlled trial of mentoring and case sheets in primary care centres," BMC Health Services Research 17(1). doi: 10.1186/s12913-016-1933-1.

11. Berhan, Yifru, and Asres Berhan. 2014. "Antenatal care as a means of increasing birth in the health facility and reducing maternal mortality: a systematic review," Ethiopian Journal of Health Sciences 24 Suppl (0 Suppl): 93-104. doi: 10.4314/ejhs.v24i0.9s.

12. Tura, Gurmesa, Mesganaw Fantahun, and Alemayehu Worku. 2013. "The effect of health facility delivery on neonatal mortality: systematic review and meta-analysis," BMC Pregnancy and Childbirth 13(18). doi: 10.1186/1471-2393-13-18.

13. Bohren, Meghan A. et al. 2014. "Facilitators and barriers to facility-based delivery in low- and middle-income countries: a qualitative evidence synthesis," Reproductive Health 11(1): 71. doi: 10.1186/1742-4755-11-71.

14. King, Rosemary, Ruth Jackson, Elaine Dietsch, and Asseffa Hailemariam. 2015. "Barriers and facilitators to accessing skilled birth attendants in Afar Region, Ethiopia," Midwifery 31(5): 540-46. doi: 10.1016/j. midw.2015.02.004.

15. Echoka, Elizabeth et al. 2013. "Existence and functionality of emergency obstetric care services at district level in Kenya: theoretical coverage versus reality," BMC Health Services Research 13: 113. doi: 10.1186/1472-6963-13-113.

16. Majrooh, Muhammad Ashraf et al. 2014. "Coverage and quality of antenatal care provided at primary health care facilities in the 'Punjab' Province of 'Pakistan,'” PLoS ONE 9(11): e113390. doi: 10.1371/ journal.pone.0113390.

17. Andrew, Erin V. W. et al. 2014. "Factors affecting attendance at and timing of formal antenatal care: results from a qualitative study in Madang, Papua New Guinea," PLOS ONE 9(5): e93025. doi: 10.1371/ journal.pone.0093025.

18. Mwaniki, Michael K. et al. 2014. "Improving service uptake and quality of care of integrated maternal health services: the Kenya Kwale District Improvement 
Collaborative," BMC Health Services Research 14(1). doi: 10.1186/1472-6963-14-416.

19. Jennings, Larissa, André Sourou Yebadokpo, Jean Affo, and Marthe Agbogbe. 2010. "Antenatal counseling in maternal and newborn care: use of job aids to improve health worker performance and maternal understanding in Benin," BMC Pregnancy and Childbirth 10(75). doi: 10.1186/1471-2393-10-75.

20. Midhet, Farid, and Stan Becker. 2010. "Impact of community-based interventions on maternal and neonatal health indicators: results from a community randomized trial in rural Balochistan, Pakistan," Reproductive Health 7(1). doi: 10.1186/1742-4755-7-30.

21. McPherson, Robert A. et al. 2010. "Process evaluation of a community-based intervention promoting multiple maternal and neonatal care practices in rural Nepal," BMC Pregnancy and Childbirth 10(31). doi: 10.1186/1471-2393-10-31.

22. McNabb, Marion et al. 2015. "Assessment of the quality of antenatal care services provided by health workers using a mobile phone decision support application in Northern Nigeria: a pre/post-intervention study," PLOS ONE 10(5): e0123940. doi: 10.1371/ journal.pone.0123940.

23. Basinga, Paulin et al. 2011. "Effect on maternal and child health services in Rwanda of payment to primary health-care providers for performance: an impact evaluation," The Lancet 377(9775): 1421-28. doi: 10.1016/S0140-6736(11)60177-3.

24. Esan, Oluwaseun T. and Adesegun O. Fatusi. 2014. "Performance needs assessment of maternal and newborn health service delivery in urban and rural areas of Osun State, South-West, Nigeria," African Journal of Reproductive Health 18(2): 105-16.

25. Taleb, Fahmida et al. 2015. "Transforming maternal and newborn health social norms and practices to increase utilization of health services in rural Bangladesh: a qualitative review," BMC Pregnancy and Childbirth 15(75). doi: 10.1186/s12884-015-0501-8.

26. Magoma, Moke et al. 2013. "The effectiveness of birth plans in increasing use of skilled care at delivery and postnatal care in rural Tanzania: a cluster randomised trial," Tropical Medicine \& International Health 18(4): 435-43. doi: 10.1111/tmi.12069.

27. Shiferaw, Solomon et al. 2013. "Why do women prefer home births in Ethiopia?," BMC Pregnancy and Childbirth 13(5). doi: 10.1186/1471-2393-13-5.
28. Dhingra, Usha et al. 2014. "Delivery, immediate newborn and cord care practices in Pemba Tanzania: a qualitative study of community, hospital staff and community level care providers for knowledge, attitudes, belief systems and practices," BMC Pregnancy and Childbirth 14(173). doi: 10.1186/1471-2393-14-173.

29. Mason, Linda et al. 2015. "Barriers and facilitators to antenatal and delivery care in Western Kenya: a qualitative study," BMC Pregnancy and Childbirth 15(26). doi: 10.1186/s12884-015-0453-z.

30. Moyer, Cheryl A. et al. 2012. "Clean delivery practices in rural northern Ghana: a qualitative study of community and provider knowledge, attitudes, and beliefs," BMC Pregnancy and Childbirth 12(50). doi: 10.1186/1471-2393-12-50.

31. Moyer, Cheryl A. et al. 2014. "'They treat you like you are not a human being': maltreatment during labour and delivery in rural Northern Ghana," Midwifery 30(2): 262-68. doi: 10.1016/j.midw.2013.05.006.

32. Ramachandran, D.L. 2010. "Mobile persuasive technologies for rural health," Doctoral dissertation, University of California, Berkley, USA. Retrieved from: https://escholarship.org/uc/item/1jk560p4.

33. Namazzi, Gertrude et al. 2015. "Strengthening health facilities for maternal and newborn care: experiences from rural Eastern Uganda," Global Health Action 8(1): 24271. doi: 10.3402/gha.v8.24271.

34. Kumar, Neha and Richard J. Anderson. 2015.

"Mobile phones for maternal health in rural India." In Proceedings of the 33rd Annual ACM Conference on Human Factors in Computing Systems (CHI '15). Association for Computing Machinery, New York, NY, USA, 427-436. doi: 10.1145/2702123.2702258

35. Bonfrer, Igna, Ellen Van de Poel, and Eddy Van Doorslaer. 2014. "The effects of performance incentives on the utilization and quality of maternal and child care in Burundi." Social Science \& Medicine 123: 96-104. doi: 10.1016/j.socscimed.2014.11.004.

36. Faye, Adama, Mariama Niane, and Ibrahim Ba. 2011. "Home birth in women who have given birth at least once in a health facility: contributory factors in a developing country," Acta Obstetricia et Gynecologica Scandinavica 90(11): 1239-43. doi: 10.1111/j.1600-0412.2011.01163.x.

37. Ratcliffe, Hannah L. et al. 2016. "Mitigating disrespect and abuse during childbirth in Tanzania: an exploratory study of the effects of two facility-based 
interventions in a large public hospital," Reproductive Health 13(1): 79. doi: 10.1186/s12978-016-0187-z.

38. van Lonkhuijzen, L, et al. 2010. "A systematic review of the effectiveness of training in emergency obstetric care in low-resource environments: systematic review of training in obstetric emergencies," BJOG: An International Journal of Obstetrics \& Gynaecology 117(7): 777-87. doi: 10.1111/j.1471-0528.2010.02561.x.

39. Gülmezoglu AM et al. 2006. "Cluster randomised trial of an active, multifaceted educational intervention based on the WHO Reproductive Health Library to improve obstetric practices," BJOG: An International Journal of Obstetrics \& Gynaecology 114(1): 16-23. doi: 10.1111/j.1471-0528.2006.01091.x.

40. van den Akker, Thomas et al. 2011. "Reduction of severe acute maternal morbidity and maternal mortality in Thyolo District, Malawi: the impact of obstetric audit," PLOS ONE 6(6): e20776. doi: 10.1371/ journal.pone.0020776.

41. Kujawski, Stephanie A. et al. 2017. "Community and health system intervention to reduce disrespect and abuse during childbirth in Tanga Region, Tanzania: a comparative before-and-after study," PLoS Medicine 14(7): e1002341. doi: 10.1371/journal.pmed.1002341.

42. Abuya, Timothy et al. 2015. "The effect of a multi-component intervention on disrespect and abuse during childbirth in Kenya," BMC Pregnancy and Childbirth 15(224). doi: 10.1186/s12884-015-0645-6.

43. Salam, Rehana A et al. 2014. "Essential childbirth and postnatal interventions for improved maternal and neonatal health," Reproductive Health 11(Suppl 1): S3. doi: 10.1186/1742-4755-11-S1-S3.

44. Mrisho, Mwifadhi et al. 2009. "the use of antenatal and postnatal care: perspectives and experiences of women and health care providers in rural Southern Tanzania," BMC Pregnancy and Childbirth 9(1). doi: 10.1186/1471-2393-9-10.

45. Mohan, Diwakar et al. 2015. "Determinants of postnatal care use at health facilities in rural Tanzania: multilevel analysis of a household survey," BMC Pregnancy and Childbirth 15(1). doi: 10.1186/ s12884-015-0717-7.

46. Jacobs, Choolwe et al. 2017. "Predictors of antenatal care, skilled birth attendance, and postnatal care utilization among the remote and poorest rural communities of Zambia: a multilevel analysis," Frontiers in Public Health 5: 11. doi: 10.3389/fpubh.2017.00011.
47. Dutamo, Zeleke, Nega Assefa, and Gudina Egata. 2015. "Maternal health care use among married women in Hossaina, Ethiopia," BMC Health Services Research 15: 1. doi: 10.1186/s12913-015-1047-1.

48. Jacobs, Choolwe et al. 2018. "Evaluation of a community-based intervention to improve maternal and neonatal health service coverage in the most rural and remote districts of Zambia," PLOS ONE 13: e0190145. doi: 10.1371/journal.pone.0190145.

49. Mwangi, Annie, Charlotte Warren, Nancy Koskei, and Holly Blanchard. 2008. "Strengthening postnatal care services including postpartum family planning in Kenya," FRONTIERS Final Report. Washington, DC: Population Council. doi: 10.31899/rh4.1181.

50. Dbaibo, Ghassan, Vladimir Tatochenko, and Peter Wutzler. 2016. "Issues in pediatric vaccine-preventable diseases in low- to middle-income countries," Human Vaccines \& Immunotherapeutics 12(9): 2365-77. doi: 10.1080/21645515.2016.1181243.

51. Ronveaux, O. et al. 2005. "The immunization data quality audit: verifying the quality and consistency of immunization monitoring systems," Bulletin of the World Health Organization 83(7): 503-10.

52. Crocker-Buque, Tim, Godwin Mindra, Richard Duncan, and Sandra Mounier-Jack. 2017. "Immunization, urbanization and slums-a systematic review of factors and interventions." BMC Public Health 17(1). doi: 10.1186/s12889-017-4473-7.

53. Organización Mundial de la Salud. 2013. Global Vaccine Action Plan 2011-2020. Geneva: World Health Organization.

54. Sridhar, Shruti et al. "A systematic literature review of missed opportunities for immunization in low- and middle-income countries," Vaccine 32(51): 6870-79. doi: 10.1016/j.vaccine.2014.10.063.

55. Cohen, Megan A. et al. 2015. "Assessing providers' vaccination behaviors during routine immunization in India," Journal of Tropical Pediatrics 61(4): 244-49. doi: 10.1093/tropej/fmv023.

56. Expanded Programme on Immunization of the Department of Immunization, Vaccines and Biologicals. 2009. "Microplanning for immunization service delivery using the Reaching Every District (RED) Strategy." Geneva: World Health Organization/ UNICEF. http://www.who.int/immunization/sage/9_ Final_RED_280909.pdf. 
57. MCHIP. N.d. "Tools and job aids for routine immunization." New Delhi: MCHIP. https://www.mchip. net/sites/default/files/revised\%20index\%20for\%20 tools\%20\&\%20job\%20aids_2.pdf.

58. Lakew, Gebre Asmamaw et al. 2017. "Status of surveillance and routine immunization performances in Amhara Region, Ethiopia: findings from in-depth peer review," Pan African Medical Journal 27. doi: 10.11604/pamj.supp.2017.27.2.10755.

59. Pradhan, Narottam et al. 2012. "Expanding and improving urban outreach immunization in Patna, India," Tropical Medicine \& International Health 17(3): 292-9. doi: 10.1111/j.1365-3156.2011.02916.x.

60. Ryman, Tove K. et al. 2011. "Implementation and evaluation of the Reaching Every District (RED) Strategy in Assam, India, 2005-2008," Vaccine 29(14): 2555-60. doi: 10.1016/j.vaccine.2011.01.061.

61. Bradley, Janet and Susan Igras. 2005. "Improving the quality of child health services: participatory action by providers," International Journal for Quality in Health Care 17(5): 391-99. doi: 10.1093/intqhc/mzi057.

62. Oliver-Williams, Clare et al. 2017. "Using mobile phones to improve vaccination uptake in 21 low- and middle-income countries: systematic review," JMIR MHealth and UHealth 5(10): e148. doi: 10.2196/ mhealth.7792.

63. Andreoni, James et al. 2016. "Using preference estimates to customize incentives: an application to polio vaccination drives in Pakistan." Cambridge, MA: National Bureau of Economic Research. doi: 10.3386/ w22019.

64. Merilind, Eero, Rauno Salupere, Katrin Västra, and Ruth Kalda. 2015. "The influence of performance-based payment on childhood immunisation coverage," Health Policy 119(6): 770-77. doi: 10.1016/j.healthpol.2015.01.015.

65. Langston, A. et al. 2014. "Plausible role for $\mathrm{CHW}$ peer support groups in increasing care-seeking in an integrated community case management project in Rwanda: a mixed methods evaluation," Global Health: Science and Practice 2(3): 342-54. doi: 10.9745/ GHSP-D-14-00067.

66. Baqui, Abdullah H. et al. 2009. "Effectiveness of home-based management of newborn infections by community health workers in rural Bangladesh," The Pediatric Infectious Disease Journal 28(4): 304-10. doi: 10.1097/INF.0b013e31819069e8.
67. Lewin, Simon et al. 2010. "Lay health workers in primary and community health care for maternal and child health and the management of infectious diseases," Cochrane Database of Systematic Reviews 2010 (3): CD004015. doi: 10.1002/14651858. CD004015.pub3.

68. Colvin, Christopher J. et al. 2013. “Understanding care-seeking for child illness in sub-Saharan Africa: a systematic review and conceptual framework based on qualitative research of household recognition and response to child diarrhoea, pneumonia and malaria," Social Science \& Medicine 86: 66-78. doi: 10.1016/j. socscimed.2013.02.031.

69. Bedford, K. Juliet A. and Alyssa B. Sharkey. "Local barriers and solutions to improve care-seeking for childhood pneumonia, diarrhea and malaria in Kenya, Nigeria and Niger: a qualitative study," PLOS ONE 9(6): e100038. doi: 10.1371/journal.pone.0100038.

70. Gilroy, Kate et al. 2006. Management of Sick Children by Community Health Workers: Intervention Models and Programme Examples. New York, NY, USA; Geneva, Switzerland: UNICEF and WHO, Dept. of Child and Adolescent Health Development.

71. Winch, Peter J. et al. 2005. "Intervention models for the management of children with signs of pneumonia or malaria by community health workers," Health Policy and Planning 20(4): 199-212. doi: 10.1093/ heapol/czi027.

72. Seidenberg, Philip D. et al. 2012. "Impact of integrated community case management on health-seeking behavior in rural Zambia," The American Journal of Tropical Medicine and Hygiene 87(Suppl 5): 105-10. doi: 10.4269/ajtmh.2012.11-0799.

73. Gilroy, K. et al. 2004. "Impact of IMCI training and language used by provider on quality of counseling provided to parents of sick children in Bougouni District, Mali," Patient Education and Counseling 54(1): 35-44. doi: 10.1016/S0738-3991(03)00189-7.

74. Carvajal-Vélez, Liliana et al. 2016. “Diarrhea management in children under five in sub-Saharan Africa: does the source of care matter? A countdown analysis," BMC Public Health 16(1). doi: 10.1186/ s12889-016-3475-1.

75. Yansaneh, Aisha I. et al. 2014. "Influence of community health volunteers on care seeking and treatment coverage for common childhood illnesses in the context of free health care in rural Sierra Leone," Tropical Medicine \& International Health 19(12): 1466-76. doi: 10.1111/tmi.12383. 
76. Chopra, Mickey et al. 2012. "Strategies to improve health coverage and narrow the equity gap in child survival, health, and nutrition," The Lancet 380(9850): 1331-40. doi: 10.1016/S0140-6736(12)61423-8.

77. Hill, Jenny et al. 2013. "Factors affecting the delivery, access, and use of interventions to prevent malaria in pregnancy in sub-Saharan Africa: a systematic review and meta-analysis," PLoS Medicine 10(7): e1001488. doi: 10.1371/journal.pmed.1001488.

78. Hill, Jenny et al. 2014. "Prioritizing pregnant women for long-lasting insecticide treated nets through antenatal care clinics," PLoS Medicine 11(9): e1001717. doi: 10.1371/journal.pmed.1001717.

79. Ezire, Onoriode et al. 2015. "Determinants of use of insecticide-treated nets among pregnant women in Nigeria," International Journal of Women's Health 7: 655-61. doi: 10.2147/IJWH.S77807.

80. Okeibunor, Joseph C. et al. 2011. "Preventing malaria in pregnancy through community-directed interventions: evidence from Akwa Ibom State, Nigeria," Malaria Journal 10(1): 227. doi: 10.1186/1475-2875-10-227.

81. Singh, Megha, Graham Brown, and Stephen J Rogerson. 2013. "Ownership and use of insecticide-treated nets during pregnancy in sub-Saharan Africa: a review," Malaria Journal 12(1): 268. doi: 10.1186/1475-2875-12-268.

82. Wang, Paul et al. 2016. "Community point distribution of insecticide-treated bed nets and community health worker hang-up visits in rural Zambia: a decision-focused evaluation." Malaria Journal 15(140). doi: 10.1186/s12936-016-1165-1.

83. Stromberg, D. G. et al. 2011. "A community health worker program for the prevention of malaria in Eastern Kenya," Education for Health 24(2): 474.

84. Maheu-Giroux, Mathieu, and Marcia C. Castro. 2014. "Factors affecting providers' delivery of intermittent preventive treatment for malaria in pregnancy: a five-country analysis of national service provision assessment surveys," Malaria Journal 13(1): 440. doi: 10.1186/1475-2875-13-440.

85. Onoka, Chima A, Obinna E. Onwujekwe, Kara Hanson, and Benjamin S. Uzochukwu. 2012. "Sub-optimal delivery of intermittent preventive treatment for malaria in pregnancy in Nigeria: influence of provider factors," Malaria Journal 11(1): 317. doi: 10.1186/1475-2875-11-317.
86. Ashwood-Smith, Hannah et al. 2002. "Availability and use of sulphadoxine-pyrimethamine (SP) in pregnancy in Blantyre District: a safe motherhood and BIMI joint survey," Malawi Medical Journal 14(1): 8-11.

87. Das, Ashis et al. 2014. "Strengthening malaria service delivery through supportive supervision and community mobilization in an endemic Indian setting: an evaluation of nested delivery models," Malaria Journal 13(1): 482. doi: 10.1186/1475-2875-13-482.

88. Ouma, P. O. et al. 2007. "The effect of health care worker training on the use of intermittent preventive treatment for malaria in pregnancy in rural Western Kenya," Tropical Medicine \& International Health 12(8): 953-61. doi: 10.1111/j.1365-3156.2007.01876.x.

89. Kabaghe, Alinune N. 2016. "Health workers' compliance to rapid diagnostic tests (RDTs) to guide malaria treatment: a systematic review and meta-analysis," Malaria Journal 15(1). doi: 10.1186/ s12936-016-1218-5.

90. Burchett, Helen E. D. et al. 2017. "Improving prescribing practices with rapid diagnostic tests (RDTs): synthesis of 10 studies to explore reasons for variation in malaria RDT uptake and adherence," BMJ Open 7(3): e012973. doi: 10.1136/bmjopen-2016-012973.

91. Aung, Tin et al. 2015. "Improving uptake and use of malaria rapid diagnostic tests in the context of artemisinin drug resistance containment in Eastern Myanmar: an evaluation of incentive schemes among informal private healthcare providers," Malaria Journal 14(1): 105. doi: 10.1186/s12936-015-0621-7.

92. Harvey, Steven A. et al. 2008. "Improving community health worker use of malaria rapid diagnostic tests in Zambia: package instructions, job aid and job aid-plus-training." Malaria Journal 7(1): 160. doi: 10.1186/1475-2875-7-160.

93. Hutchinson, Eleanor et al. 2015. "'It puts life in us and we feel big': shifts in the local health care system during the introduction of rapid diagnostic tests for malaria into drug shops in Uganda," Critical Public Health 25(1): 48-62. doi: 10.1080/09581596.2014.886762.

94. Wahlström, Rolf et al. 2003. "Effectiveness of feedback for improving case management of malaria, diarrhoea and pneumonia-a randomized controlled trial at provincial hospitals in Lao PDR," Tropical Medicine \& International Health 8 (10): 901-9. doi: 10.1046/j.1365-3156.2003.01105.x. 
95. Cundill, Bonnie et al. 2015. "Prescriber and patient-oriented behavioural interventions to improve use of malaria rapid diagnostic tests in Tanzania: facility-based cluster randomised trial," BMC Medicine 13(1). doi: 10.1186/s12916-015-0346-z.

96. Reyburn, Hugh et al. 2004. "Overdiagnosis of malaria in patients with severe febrile illness in Tanzania: a prospective study," BMJ 329(7476): 1212. doi: 10.1136/bmj.38251.658229.55.

97. Sudoi, Raymond K. et al. 2012. "The magnitude and trend of Artemether-Lumefantrine Stock-Outs at public health facilities in Kenya," Malaria Journal 11(1): 37. doi: 10.1186/1475-2875-11-37.

98. Whitty, Christopher JM et al. 2008. "Deployment of ACT antimalarials for treatment of malaria: challenges and opportunities," Malaria Journal 7(Suppl 1): S7. doi: 10.1186/1475-2875-7-S1-S7.

99. Wasunna, Beatrice, Dejan Zurovac, Catherine A Goodman, and Robert W Snow. 2008. "Why don't health workers prescribe ACT? a qualitative study of factors affecting the prescription of ArtemetherLumefantrine," Malaria Journal 7(1): 29. doi: 10.1186/1475-2875-7-29.

100. O'Connell, Kathryn A. et al. 2011. "Got ACTs? Availability, price, market share and provider knowledge of anti-malarial medicines in public and private sector outlets in six malaria-endemic countries," Malaria Journal 10(1): 326. doi: 10.1186/1475-2875-10-326.

101. Selemani, Majige et al. 2013. "Health worker factors associated with prescribing of artemisinin combination therapy for uncomplicated malaria in rural Tanzania," Malaria Journal 12(1): 334. doi: 10.1186/1475-2875-12-334.

102. Rowe, Alexander K., Faustin Onikpo, Marcel Lama, and Michael S. Deming. 2003. "Risk and protective factors for two types of error in the treatment of children with fever at outpatient health facilities in Benin," International Journal of Epidemiology 32(2): 296-303.

103. Dodoo, Alexander N.O. et al. 2009. "Pattern of drug utilization for treatment of uncomplicated malaria in urban Ghana following national treatment policy change to artemisinin-combination therapy," Malaria Journal 8(1): 2. doi: 10.1186/1475-2875-8-2.

104. Bawate, Charles, Sylvia T. Callender-Carter, Ben Nsajju, and Denis Bwayo. 2016. "Factors affecting adherence to national malaria treatment guidelines in management of malaria among public healthcare workers in
Kamuli District, Uganda," Malaria Journal 15(1). doi: 10.1186/s12936-016-1153-5.

105. Hamer, Davidson H. et al. 2007. "Improved diagnostic testing and malaria treatment practices in Zambia," JAMA 297(20): 2227. doi: 10.1001/jama.297.20.2227.

106. Chandler, Clare I.R. et al. 2008. "Guidelines and mindlines: why do clinical staff over-diagnose malaria in Tanzania? a qualitative study," Malaria Journal 7(1): 53. doi: 10.1186/1475-2875-7-53.

107. Smith, Lucy A., Caroline Jones, Sylvia Meek, and Jayne Webster. 2009. "Review: provider practice and user behavior interventions to improve prompt and effective treatment of malaria: do we know what works?," The American Journal of Tropical Medicine and Hygiene 80(3): 326-35.

108. Armstrong Schellenberg, Joanna et al. 2004. "The effect of integrated management of childhood illness on observed quality of care of under-fives in rural Tanzania," Health Policy and Planning 19(1): 1-10.

109. World Health Organization and UNICEF. 1997. "IMCl In-Service Training." Geneva: World Health Organization/UNICEF. http://www.who.int/maternal_child_adolescent/documents/9241595650/ en/.

110. Gouws, Eleanor et al. 2004. "Improving antimicrobial use among health workers in first-level facilities: results from the multi-country evaluation of the integrated management of childhood illness strategy," Bulletin of the World Health Organization 82(7): 509-15.

111. Zurovac, D. et al. 2004. "Predictors of the quality of health worker treatment practices for uncomplicated malaria at government health facilities in Kenya," International Journal of Epidemiology 33(5): 1080-91. doi: 10.1093/ije/dyh253.

112. Masanja, Irene M. et al. 2013. "Correct dosing of artemether-lumefantrine for management of uncomplicated malaria in rural Tanzania: do facility and patient characteristics matter?" Malaria Journal 12(1): 446. doi: 10.1186/1475-2875-12-446.

113. Wasunna, Beatrice et al. 2010. "Health worker performance in the management of pediatric fevers following in-service training and exposure to job aids in Kenya," Malaria Journal 9(1): 261. doi: 10.1186/1475-2875-9-261.

114. Rowe, A. K. et al. 2000. "Predictors of correct treatment of children with fever seen at outpatient health 
facilities in the Central African Republic," American Journal of Epidemiology 151(10): 1029-35.

115. Ofori-Adjei, D., and D. K. Arhinful. 1996. "Effect of training on the clinical management of malaria by medical assistants in Ghana," Social Science \& Medicine 42(8): 1169-76.

116. Littrell, Megan et al. 2011. "Monitoring fever treatment behaviour and equitable access to effective medicines in the context of initiatives to improve ACT access: baseline results and implications for programming in six African countries," Malaria Journal 10(1): 327. doi: 10.1186/1475-2875-10-327.

117. Bello, Danjuma Ayotunde et al. 2013. "Supportive supervision: an effective intervention in achieving high quality malaria case management at primary health care level in Jos, Nigeria," Annals of African Medicine 12(4): 243. doi: 10.4103/1596-3519.122695.

118. Osterholt, Dawn M. et al. 2006. "Predictors of treatment error for children with uncomplicated malaria seen as outpatients in Blantyre District, Malawi," Tropical Medicine and International Health 11(8): 1147-56. doi: 10.1111/j.1365-3156.2006.01666.x.

119. Zurovac, Dejan et al. 2011. "The effect of mobile phone text-message reminders on Kenyan health workers' adherence to malaria treatment guidelines: a cluster randomised trial," The Lancet 378(9793): 795-803. doi: 10.1016/S0140-6736(11)60783-6.

120. Yeboah-Antwi, K. et al. 2001. "Impact of prepackaging antimalarial drugs on cost to patients and compliance with treatment," Bulletin of the World Health Organization 79(5): 394-99.

121. Pagnoni, F. et al. 1997. "A community-based programme to provide prompt and adequate treatment of presumptive malaria in children," Transactions of the Royal Society of Tropical Medicine and Hygiene 91(5): 512-17.

122. Gidebo, K D, Thandisizwe Redford Mavundla, and T E Masango. 2015. "Factors influencing malaria treatment and patient adherence to antimalarial drugs in Southern Ethiopia," Africa Journal of Nursing and Midwifery 16(2): 85. doi: 10.25159/2520-5293/39.

123. Meremikwu, Martin et al. 2007. "Antimalarial drug prescribing practice in private and public health facilities in South-East Nigeria: a descriptive study," Malaria Journal 6(1): 55. doi: 10.1186/1475-2875-6-55.

124. Debes, Amanda K. 2013. "Time to initiation of breastfeeding and neonatal mortality and morbidity: a systematic review," BMC Public Health 13(Suppl 3): S19. doi: 10.1186/1471-2458-13-S3-S19.

125. Khan, Jehangir, Linda Vesel, Rajiv Bahl, and José Carlos Martines. 2015. "Timing of breastfeeding initiation and exclusivity of breastfeeding during the first month of life: effects on neonatal mortality and morbidity-a systematic review and meta-analysis," Maternal and Child Health Journal 19(3): 468-79. doi: 10.1007/ s10995-014-1526-8.

126. Sharma, Arvind et al. 2016. "Factors associated with early initiation of breastfeeding among mothers of tribal area of Madhya Pradesh, India: a community based cross sectional study," International Journal of Community Medicine and Public Health 3(1): 194-99. doi: 10.18203/2394-6040.ijcmph20151561.

127. Setegn, Tesfaye, Mulusew Gerbaba, and Tefera Belachew. 2011. "Determinants of timely initiation of breastfeeding among mothers in Goba Woreda, South East Ethiopia: a cross sectional study," BMC Public Health 11(217). doi: 10.1186/1471-2458-11-217.

128. Singh, Kavita et al. 2017. "The importance of skinto-skin contact for early initiation of breastfeeding in Nigeria and Bangladesh," Journal of Global Health 7(2): 020505. doi: 10.7189/jogh.07.020505.

129. Baker, Elizabeth Jean, Linda C. Sanei, and Nadra Franklin. 2006. "Early initiation of and exclusive breastfeeding in large-scale community-based programmes in Bolivia and Madagascar," Journal of Health, Population, and Nutrition 24(4): 530-39.

130. Sharma, Indu K., and Abbey Byrne. 2016. "Early initiation of breastfeeding: a systematic literature review of factors and barriers in South Asia," International Breastfeeding Journal 11(1). doi: 10.1186/ s13006-016-0076-7.

131. Legesse, Misgan, Melake Demena, Firehiwot Mesfin, and Demewoz Haile. 2014. "Prelacteal feeding practices and associated factors among mothers of children aged less than 24 months in Raya Kobo District, North Eastern Ethiopia: a cross-sectional study," International Breastfeeding Journal 9(1): 189. doi: 10.1186/s13006-014-0025-2.

132. Savage, F., and B. Daelmans. 1995. "Training health care workers to counsel breastfeeding mothers," Dialogue on Diarrhoea 59: 2.

133. Rea, M. F., S. I. Venancio, J. C. Martines, and F. Savage. 1999. "Counselling on breastfeeding: assessing knowledge and skills," Bulletin of the World Health Organization 77(6): 492-98. 
134. Sinha, Bireshwar, et al. 2015. "Interventions to improve breastfeeding outcomes: a systematic review and meta-analysis," Acta Paediatrica 104: 114-34. doi: 10.1111/apa.13127.

135. Marx Delaney, Megan et al. 2017. "Improving adherence to essential birth practices using the WHO Safe Childbirth Checklist with peer coaching: experience from 60 public health facilities in Uttar Pradesh, India," Global Health: Science and Practice 5(2): 217-31. doi: 10.9745/GHSP-D-16-00410.

136. Kramer, Michael S, and Ritsuko Kakuma. 2012. "Optimal duration of exclusive breastfeeding," Cochrane Database of Systematic Reviews 8: CD003517. doi: 10.1002/14651858.CD003517.pub2.

137. Wouk, Kathryn et al. 2016. "Clinical interventions to promote breastfeeding by Latinas: a meta-analysis," Pediatrics 137(1): e20152423. doi: 10.1542/ peds.2015-2423.

138. Aidam, Bridget A., Rafael Pérez-Escamilla, and Anna Lartey. 2005. "Lactation counseling increases exclusive breast-feeding rates in Ghana," The Journal of Nutrition 135(7): 1691-95. doi: 10.1093/jn/135.7.1691.

139. Morrow, Ardythe L. et al. 1999. "Efficacy of homebased peer counselling to promote exclusive breastfeeding: a randomised controlled trial," The Lancet 353(9160): 1226-31. doi: 10.1016/ s0140-6736(98)08037-4.

140. Tylleskär, Thorkild et al. 2011. "Exclusive breastfeeding promotion by peer counsellors in sub-Saharan Africa (PROMISE-EBF): a cluster-randomised trial," The Lancet 378(9789): 420-27. doi: 10.1016/ s0140-6736(11)60738-1.

141. Haroon, Sarah et al. 2013. "Breastfeeding promotion interventions and breastfeeding practices: a systematic review," BMC Public Health 13(Suppl 3): S20. doi: 10.1186/1471-2458-13-S3-S20.

142. Balogun, Olukunmi et al. 2015. "Factors influencing breastfeeding exclusivity during the first 6 months of life in developing countries: a quantitative and qualitative systematic review," Maternal \& Child Nutrition 11(4): 433-51. doi: 10.1111/mcn.12180.

143. Guyon, Agnès B. et al. 2009. "Implementing an integrated nutrition package at large scale in Madagascar: the essential nutrition actions framework," Food and Nutrition Bulletin 30(3): 233-44. doi: 10.1177/156482650903000304.
144. Syed, Uzma et al. 2006. "Immediate and early postnatal care for mothers and newborns in rural Bangladesh," Journal of Health, Population, and Nutrition 24(4): 508-18.

145. Robinson, S. M. 2015. "Infant nutrition and lifelong health: current perspectives and future challenges," Journal of Developmental Origins of Health and Disease 6(05): 384-89. doi: 10.1017/ S2040174415001257.

146. Imdad, Aamer, Mohammad Yawar Yakoob, and Zulfiqar A Bhutta. 2011. "Impact of maternal education about complementary feeding and provision of complementary foods on child growth in developing countries," BMC Public Health 11(Suppl 3): S25. doi: 10.1186/1471-2458-11-S3-S25.

147. Penny, Mary E. et al. 2005. "Effectiveness of an educational intervention delivered through the health services to improve nutrition in young children: a cluster-randomised controlled trial," The Lancet 365(9474): 1863-72. doi: 10.1016/ s0140-6736(05)66426-4.

148. Shi, Ling et al. 2010. "Effectiveness of an educational intervention on complementary feeding practices and growth in rural China: a cluster randomised controlled trial," Public Health Nutrition 13(4): 556-65. doi: 10.1017/S1368980009991364

149. Guldan, G. S. et al. 2000. "Culturally appropriate nutrition education improves infant feeding and growth in rural Sichuan, China." The Journal of Nutrition 130(5): 1204-11. doi: 10.1093/jn/130.5.1204.

150. Roy, S. K. et al. 2005. "Intensive nutrition education with or without supplementary feeding improves the nutritional status of moderately-malnourished children in Bangladesh," Journal of Health, Population, and Nutrition 23(4): 320-30.

151. Bhandari, N. et al. 2001. "Food supplementation with encouragement to feed it to infants from 4 to 12 months of age has a small impact on weight gain," The Journal of Nutrition 131(7): 1946-51. doi: 10.1093/ jn/131.7.1946.

152. Santos, I. et al. 2001. "Nutrition counseling increases weight gain among Brazilian children," The Journal of Nutrition 131(11): 2866-73. doi: 10.1093/ jn/131.11.2866.

153. El-Sayed, Hesham et al. 2014. "The effectiveness of the WHO training course on complementary feeding counseling in a primary care setting, Ismailia, Egypt," The Journal of the Egyptian Public Health Association 
89(1): 1-8. doi: 10.1097/01.EPX.0000443990.46047. a6.

154. Weltgesundheitsorganisation, ed. 2003. Global Strategy for Infant and Young Child Feeding. Geneva: WHO.

155. Sunguya, Bruno F. et al. 2013. "Nutrition training improves health workers' nutrition knowledge and competence to manage child undernutrition: a systematic review," Frontiers in Public Health 1. doi: 10.3389/fpubh.2013.00037.

156. Pelto, Gretel H. et al. 2004. "Nutrition counseling training changes physician behavior and improves caregiver knowledge acquisition," The Journal of Nutrition 134(2): 357-62. doi: 10.1093/jn/134.2.357.

157. Zaman, Shakila, Rifat N. Ashraf, and José Martines. 2008. "Training in complementary feeding counselling of healthcare workers and its influence on maternal behaviours and child growth: a cluster-randomized controlled trial in Lahore, Pakistan." Journal of Health, Population, and Nutrition 26(2): 210-22.

158. Kennedy, Caitlin E. et al. 2013. "Provider-initiated HIV testing and counseling in low- and middle-income countries: a systematic review," AIDS and Behavior 17(5): 1571-90. doi: 10.1007/s10461-012-0241-y.

159. World Health Organization, and Joint United Nations Programme on HIV/AIDS. 2007. Guidance on ProviderInitiated HIV Testing and Counselling in Health Facilities. Geneva: World Health Organization.

160. Creek, Tracy L. et al. 2007. "Successful introduction of routine opt-out HIV testing in antenatal care in Botswana," Journal of Acquired Immune Deficiency Syndromes 45(1): 102-7. doi: 10.1097/ QAI.0b013e318047df88.

161. Homsy, Jaco et al. 2006. "Routine intrapartum HIV counseling and testing for prevention of motherto-child transmission of HIV in a rural Ugandan hospital," Journal of Acquired Immune Deficiency Syndromes 42(2): 149-54. doi: 10.1097/01. qai.0000225032.52766.c2.

162. Chandisarewa, Winfreda et al. 2007. "Routine offer of antenatal HIV testing ('opt-out' approach) to prevent mother-to-child transmission of HIV in urban Zimbabwe," Bulletin of the World Health Organization 85(11): 843-50. doi: 10.2471/BLT.06.035188.

163. Evans, Catrin, and Eunice Ndirangu. 2011. "Implementing routine provider-initiated HIV testing in public health care facilities in Kenya: a qualitative descriptive study of nurses' experiences," AIDS Care 23(10): 1291-97. doi: 10.1080/09540121.2011.555751.

164. World Health Organization. 2015. Consolidated Guidelines on HIV Testing Services: 5Cs: Consent, Confidentiality, Counselling, Correct Results, and Connection. Geneva: World Health Organization. http://www.ncbi.nlm.nih.gov/books/NBK316021/.

165. Bott, Sarah et al. 2015. "Rewards and challenges of providing HIV testing and counselling services: health worker perspectives from Burkina Faso, Kenya and Uganda," Health Policy and Planning 30(8): 964-75. doi: 10.1093/heapol/czu100.

166. Roura, Maria et al. 2013. "Provider-initiated testing and counselling programmes in sub-Saharan Africa: a systematic review of their operational implementation," AIDS 27(4): 617-26. doi: 10.1097/ QAD.0b013e32835b7048.

167. MacPherson, Peter et al. 2012. "Suboptimal patterns of provider initiated HIV testing and counselling, antiretroviral therapy eligibility assessment and referral in primary health clinic attendees in Blantyre, Malawi," Tropical Medicine \& International Health 17(4): 507-17. doi: 10.1111/j.1365-3156.2011.02946.x.

168. Medley, Amy M., and Caitlin E. Kennedy. 2010. "Provider challenges in implementing antenatal provider-initiated HIV testing and counseling programs in Uganda," AIDS Education and Prevention 22(2): 87-99. doi: 10.1521/aeap.2010.22.2.87.

169. Ahmed, Shahira et al. 2016. "How providers influence the implementation of provider-initiated HIV testing and counseling in Botswana: a qualitative study," Implementation Science 11. doi: 10.1186/ s13012-015-0361-7.

170. World Health Organization. "HIV/AIDS training." Accessed 15 July, 2018. http://www.who.int/hiv/topics/vct/toolkit/components/training/en/index4.html.

171. de Walque, Damien et al. 2015. "Using provider performance incentives to increase HIV testing and counseling services in Rwanda," Journal of Health Economics 40: 1-9. doi: 10.1016/j. jhealeco.2014.12.001

172. Pulerwitz, Julie et al. 2010. "Reducing HIV-related stigma: lessons learned from Horizons research and programs," Public Health Reports 125(2): 272-81. doi: 10.1177/003335491012500218

173. Mahendra, Vaishali S. et al. 2006. "Reducing AIDSrelated stigma and discrimination in Indian hospitals," 
Horizons Final Report. New Delhi: Population Council. doi: 10.31899/HIV2.1027

174. Pulerwitz, Julie et al. 2015. "Improving hospital-based quality of care by reducing HIV-related stigma: evaluation results from Vietnam," AIDS and Behavior 19(2): 246-56. doi: 10.1007/s10461-014-0935-4.

175. Evans, Catrin et al. 2016. "The views and experiences of nurses and midwives in the provision and management of provider-initiated HIV testing and counseling: a systematic review of qualitative evidence," JBI Database of Systematic Reviews and Implementation Reports 13(12): 130-286. doi: 10.11124/jbisrir-2015-2345.

176. Nyblade, Laura, Anne Stangl, Ellen Weiss, and Kim Ashburn. 2009. "Combating HIV stigma in health care settings: what works?" Journal of the International AIDS Society 12: 15. doi: 10.1186/1758-2652-12-15.

177. Labhardt, Niklaus Daniel et al. 2014. "Home-based versus mobile clinic HIV testing and counseling in rural Lesotho: a cluster-randomized trial." PLOS Medicine 11(12): e1001768. doi: 10.1371/journal. pmed.1001768.

178. Mwai, Grace W. et al. 2013. "Role and outcomes of community health workers in HIV care in sub-Saharan Africa: a systematic review," Journal of the International AIDS Society 16: 18586. doi: 10.7448/ IAS.16.1.18586

179. Clinical and Community HIV/AIDS Services Strengthening (CHASS) project. 2017. "Implementation of community index case testing," Fact sheet. Washington, DC: FHI 360. Accessed 11 April 2018. https://www.fhi360.org/resource/implementation-community-index-case-testing-fact-sheet.

180. Zachariah, R. et al. 2006. "How can the community contribute in the fight against HIV/AIDS and tuberculosis? an example from a rural district in Malawi," Transactions of the Royal Society of Tropical Medicine and Hygiene 100(2): 167-75. doi: 10.1016/j. trstmh.2005.07.008.

181. Jean-Baptiste, Rachel. 2008. "HIV/AIDS-related stigma, fear, and discriminatory practices among healthcare providers in Rwanda," Operations Research Results. Published for the U.S. Agency for International Development (USAID) by the Quality Assurance Project. Bethesda, MD: University Research Co., LLC.

182. World Health Organization. 2015. "WHO recommendations to assure HIV testing quality." Geneva: World Health Organization.
183. Yao, Katy et al. 2010. "Ensuring the quality of HIV rapid testing in resource-poor countries using a systematic approach to training," American Journal of Clinical Pathology 134(4): 568-72. doi: 10.1309/ AJCPOPXR8MNTZ5PY.

184. Eaton, Jeffrey W., Cheryl C. Johnson, and Simon Gregson. 2017. "The cost of not retesting: human immunodeficiency virus misdiagnosis in the antiretroviral therapy 'test-and-offer' era," Clinical Infectious Diseases 65(3): 522-25. doi: 10.1093/cid/cix341

185. Kanal, Koum et al. 2005. "Evaluation of the proficiency of trained non-laboratory health staffs and laboratory technicians using a rapid and simple HIV antibody test," AIDS Research and Therapy 2: 5. doi: 10.1186/1742-6405-2-5.

186. Kennedy, C. E., P. T. Yeh, C. Johnson, and R. Baggaley. 2017. "Should trained lay providers perform HIV testing? A systematic review to inform World Health Organization guidelines," AIDS Care 29(12): 1473-79. doi: 10.1080/09540121.2017.1317710.

187. Mwangala, Sheila et al. 2016. "Accuracy in HIV rapid testing among laboratory and non-laboratory personnel in Zambia: observations from the National HIV Proficiency Testing System," PLOS ONE 11(1): e0146700. doi: 10.1371/journal.pone.0146700.

188. Parekh, Bharat S. et al. 2010. "Scaling up HIV rapid testing in developing countries: comprehensive approach for implementing quality assurance," American Journal of Clinical Pathology 134(4): 573-84. doi: 10.1309/AJCPTDIMFROOIKYX.

189. Learmonth, Kate M. et al. 2008. "Assessing proficiency of interpretation of rapid human immunodeficiency virus assays in nonlaboratory settings: ensuring quality of testing," Journal of Clinical Microbiology 46(5): 1692-97. doi: 10.1128/JCM.01761-07.

190. Kim, Young Mi et al. 2013. "Evaluation of a quality improvement intervention to prevent mother-to-child transmission of HIV (PMTCT) at Zambia Defence Force facilities," BMC Health Services Research 13(1). doi: 10.1186/1472-6963-13-345.

191. Hogben, Matthew, Tarra McNally, Melissa McPheeters, and Angela B. Hutchinson. 2007. "The effectiveness of HIV partner counseling and referral services in increasing identification of HIV-positive individuals a systematic review," American Journal of Preventive Medicine 33(Suppl 2): S89-100. doi: 10.1016/j.amepre.2007.04.015. 
192. Passin, Warren F. et al. 2006. "A systematic review of HIV partner counseling and referral services: client and provider attitudes, preferences, practices, and experiences," Sexually Transmitted Diseases 33(5): 320-28. doi: 10.1097/01.olq.0000194597.16236.48.

193. World Health Organization. 2016. Guidelines on HIV Self-Testing and Partner Notification: Supplement to Consolidated Guidelines on HIV Testing Services. Geneva: World Health Organization.

194. Netsanet, Fetene, and Ayalew Dessie. 2013.

"Acceptance of referral for partners by clients testing positive for human immunodeficiency virus," HIV/ AIDS-Research and Palliative Care 5: 19-28. doi: 10.2147/HIV.S39250.

195. World Health Organization. 2015. Guideline on When to Start Antiretroviral Therapy and on PreExposure Prophylaxis for HIV. Geneva: World Health Organization. http://www.ncbi.nlm.nih.gov/books/ NBK327115/.

196. Nsigaye, Ray et al. 2009. "From HIV diagnosis to treatment: evaluation of a referral system to promote and monitor access to antiretroviral therapy in rural Tanzania," Journal of the International AIDS Society 12: 31. doi: 10.1186/1758-2652-12-31.

197. Department of Health and Human Services Panel on Antiretroviral Guidelines for Adults and Adolescents. "Initiation of antiretroviral therapy adult and adolescent ARV." Washington, DC: AIDSinfo. Accessed 12 June 2018. https://aidsinfo.nih.gov/ guidelines/html/1/adult-and-adolescent-arv/10/ initiation-of-antiretroviral-therapy.

198. Kalichman, Seth C. et al. 2010. "Adherence to antiretroviral therapy and HIV transmission risks: implications for test-and-treat approaches to HIV prevention," AIDS Patient Care and STDs 24(5): 271-77. doi: 10.1089/apc.2009.0309.

199. Rosen, Sydney, and Matthew P. Fox. 2011. "Retention in HIV care between testing and treatment in sub-Saharan Africa: a systematic review," PLOS Medicine 8(7): e1001056. doi: 10.1371/journal.pmed.1001056.

200. Labhardt, Niklaus Daniel et al. 2016. "Same day ART initiation versus clinic-based pre-ART assessment and counselling for individuals newly tested HIVpositive during community-based HIV testing in rural Lesotho-a Randomized Controlled Trial (CASCADE Trial)," BMC Public Health 16: 329. doi: 10.1186/ s12889-016-2972-6.
201. Govindasamy, Darshini, Nathan Ford, and Katharina Kranzer. 2012. "Risk factors, barriers and facilitators for linkage to antiretroviral therapy care: a systematic review," AIDS 26(16): 2059-67. doi: 10.1097/ QAD.0b013e3283578b9b.

202. Bendavid, Eran, Margaret L. Brandeau, Robin Wood, and Douglas K. Owens. 2010. "Comparative effectiveness of HIV testing and treatment in highly endemic regions," Archives of Internal Medicine 170(15): 1347-54. doi: 10.1001/archinternmed.2010.249.

203. Uebel, Kerry E. et al. 2011. "Task shifting and integration of HIV care into primary care in South Africa: the development and content of the Streamlining Tasks and Roles to Expand Treatment and Care for HIV (STRETCH) intervention," Implementation Science 6(1). doi: 10.1186/1748-5908-6-86.

204. Fairall, Lara et al. 2012. "Task shifting of antiretroviral treatment from doctors to primary-care nurses in South Africa (STRETCH): a pragmatic, parallel, cluster-randomised trial," The Lancet 380(9845): 889-98. doi: 10.1016/S0140-6736(12)60730-2.

205. Kieffer, Mary Pat et al. 2014. "Lessons learned from early implementation of Option B+: The Elizabeth Glaser Pediatric AIDS Foundation experience in 11 African countries," Journal of Acquired Immune Deficiency Syndromes 67(Suppl 4): S188-94. doi: 10.1097/QAI.0000000000000372.

206. Zwarenstein, Merrick et al. 2011. "Outreach education for integration of HIV/AIDS care, antiretroviral treatment, and tuberculosis care in primary care clinics in South Africa: PALSA PLUS Pragmatic Cluster Randomised Trial," The BMJ 342. doi: 10.1136/bmj. d2022.

207. Chang, Larry William et al. 2012. "Evaluation of a multimodal, distance learning HIV management course for clinical care providers in India," Journal of the International Association of Physicians in AIDS Care 11(5): 277-82. doi: 10.1177/1545109712451330.

208. MacPherson, Peter et al. 2014. "A novel community health worker tool outperforms WHO clinical staging for assessment of antiretroviral therapy eligibility in a resource-limited setting," Journal of Acquired Immune Deficiency Syndromes 65(2): e74-78. doi: 10.1097/ QAI.0b013e3182a20e74.

209. Weiser, John et al. 2017. "Barriers to universal prescribing of antiretroviral therapy by HIV care providers in the United States, 2013-2014," Journal of Acquired Immune Deficiency Syndromes 74(5): 479-87. doi: 10.1097/QAI.0000000000001276. 
210. World Health Organization. 2017. "What's new in treatment monitoring: viral load and CD4 testing." Geneva: World Health Organization. https://www.who.int/hiv/pub/arv/ treatment-monitoring-info-2017/en/

211. UNAIDS. 2016. "The need for routine viral load testing: questions and answers." Geneva: UNAIDS. https://www.unaids.org/sites/default/files/ media_asset/JC2845_en.pdf

212. Dorward, Jienchi, Paul K. Drain, and Nigel Garrett. 2018. "Point-of-care viral load testing and differentiated HIV care," The Lancet. HIV 5(1): e8-9. doi: 10.1016/S2352-3018(17)30211-4.

213. Phillips, Andrew N. et al. 2016. "Point-of-care viral load testing for sub-Saharan Africa: informing a target product profile," Open Forum Infectious Diseases 3(3). doi: 10.1093/ofid/ofw161.

214. Bonner, Kimberly et al. 2013. "Viral load monitoring as a tool to reinforce adherence: a systematic review," Journal of Acquired Immune Deficiency Syndromes 64(1): 74-78. doi: 10.1097/QAl.0b013e31829f05ac.

215. ART-LINC of leDEA Study Group et al. 2009. "Switching to second-line antiretroviral therapy in resource-limited settings: comparison of programmes with and without viral load monitoring," AIDS 23(14): 1867-74. doi: 10.1097/QAD.0b013e32832e05b2.

216. Médecins Sans Frontières Access Campaign. 2014. "Getting to undetectable: usage of HIV viral load monitoring in five countries." Geneva: Médecins Sans Frontières Access Campaign.

217. Roberts, Teri, Jennifer Cohn, Kimberly Bonner, and Sally Hargreaves. 2016. "Scale-up of routine viral load testing in resource-poor settings: current and future implementation challenges," Clinical Infectious Diseases 62(8): 1043-48. doi: 10.1093/cid/ciw001.

218. Carmona, Sergio, Trevor Peter, and Leigh Berrie. 2017. "HIV viral load scale-up: multiple interventions to meet the HIV treatment cascade," Current Opinion in HIV and AIDS 12(2): 157-64. doi: 10.1097/ $\mathrm{COH} .0000000000000352$.

219. World Health Organization. 2014. "Technical and operational considerations for implementing HIV viral load testing." Geneva: World Health Organization.

220. Workneh, Gelane et al. 2013. "Evaluation of the effectiveness of an outreach clinical mentoring programme in support of paediatric HIV care scale-up in Botswana," AIDS Care 25(1): 11-19. doi: 10.1080/09540121.2012.674096.

221. Swannet, Sarah et al. 2017. "Journey towards universal viral load monitoring in Maputo, Mozambique: many gaps, but encouraging signs," International Health 9(4): 206-14. doi: 10.1093/inthealth/ihx021.

222. Médecins Sans Frontières. 2016. "Making viral load routine. part 1: programmatic strategies." Geneva: Médecins Sans Frontières.

223. Bangsberg, D. R. et al. 2001. "Provider assessment of adherence to HIV antiretroviral therapy," Journal of Acquired Immune Deficiency Syndromes 26(5): 435-42.

224. Lecher, Andrew F. et al. 2015. "Scale-up of HIV viral load monitoring - seven sub-Saharan African countries," Morbidity and Mortality Weekly Report 64(46): 1281-86. doi: 10.15585/mmwr.mm6446a2.

225. El-Sadr, Wafaa M, Miriam Rabkin, John Nkengasong, and Deborah L Birx. 2017. "Realizing the potential of routine viral load testing in sub-Saharan Africa," Journal of the International AIDS Society 20 (Suppl 7): e25010. doi: 10.1002/jia2.25010.

226. The Organization for Public Health Interventions and Development. 2016. "Viral load monitoring and the last 90: VL snapshot." http://www.ophid.org/ sites/default/files/Viral\%20Load\%20Monitoring\%20 and\%20the\%203rd\%2090-min.pdf

227. Horter, Shona et al. 2015. "Viral load monitoring: how do HIV-positive patients interpret and understand their results?," Presented at Médecins Sans Frontières (MSF) Scientific Day 2015, London, 7-8 May.

228. Gibb, Jean et al. 2017. "Supporting quality data systems: lessons learned from early implementation of routine viral load monitoring at a large clinic in Lilongwe, Malawi," Journal of Clinical Research in HIV AIDS and Prevention 3(1). doi: 10.14302/issn.23247339.jcrhap-17-1468

229. Kandulu, James. 2016. "HIV viral load supply chain management and development during scale-up." Presented at International AIDS Conference, Durban, South Africa, 21 July.

230. Médecins Sans Frontières and Southern Africa Medical Unit. "Viral load monitoring" Accessed 14 June 2018. https://samumsf.org/en/resources/hiv/ viral-load-monitoring. 
231. Kagee, A. et al. 2011. "Structural barriers to ART adherence in Southern Africa: challenges and potential ways forward," Global Public Health 6(1): 83-97. doi: 10.1080/17441691003796387.

232. Jobanputra, Kiran et al. 2014. "Impact and programmatic implications of routine viral load monitoring in Swaziland," Journal of Acquired Immune Deficiency Syndromes 67(1): 45-51. doi: 10.1097/ QAI.0000000000000224.

233. Médecins Sans Frontières Analysis \& Advocacy Unit and Southern Africa Medical Unit. "HIV/TB counselling: who is doing the job?" Cape Town, South Africa: Medecines Sans Frontières.

234. Wilson, David, et al. 2009. "HIV-1 viral load monitoring: an opportunity to reinforce treatment adherence in a resource-limited setting in Thailand," Transactions of The Royal Society of Tropical Medicine and Hygiene 103(6): 601-6. doi: 10.1016/j.trstmh.2008.11.007.

235. Beach, Mary Catherine, Jeanne Keruly, and Richard D Moore. "Is the quality of the patient-provider relationship associated with better adherence and health outcomes for patients with HIV?," Journal of General Internal Medicine 21(6): 661-65. doi: 10.1111/j.1525-1497.2006.00399.x.

236. Denno, Donna M., Andrea J. Hoopes, and Venkatraman Chandra-Mouli. 2015. "Effective strategies to provide adolescent sexual and reproductive health services and to increase demand and community support," Journal of Adolescent Health 56(1): S22-41. doi: 10.1016/j.jadohealth.2014.09.012.

237. Pathfinder International. 2017. "Mainstreaming youth-friendly sexual \& reproductive health services in the public sector in Mozambique \& Tanzania." Pathfinder International. http://www.pathfinder. org/publications/mainstreaming-youth-friendly-services-mozambique-tanzania/.

238. Chandra-Mouli, Venkatraman, Catherine Lane, and Sylvia Wong. 2015. "What does not work in adolescent sexual and reproductive health: a review of evidence on interventions commonly accepted as best practices," Global Health: Science and Practice 3(3): 333-40. doi: 10.9745/GHSP-D-15-00126.

239. Gottschalk, Lindsey B., and Nuriye Ortayli. 2014. "Interventions to improve adolescents' contraceptive behaviors in low- and middle-income countries: a review of the evidence base," Contraception 90(3): 211-25. doi: 10.1016/j.contraception.2014.04.017.
240. Kesterton, Amy J., and Meena Cabral de Mello. "Generating demand and community support for sexual and reproductive health services for young people: a review of the literature and programs," Reproductive Health 7: 25. doi: 10.1186/1742-4755-7-25.

241. Wood, Kate, and Rachel Jewkes. 2006. "Blood blockages and scolding nurses: barriers to adolescent contraceptive use in South Africa." Reproductive Health Matters 14(27). https://www.tandfonline.com/ doi/full/10.1016/S0968-8080\%2806\%2927231-8.

242. Bastien, S., L. J. Kajula, and W. W. Muhwezi. 2011. "A review of studies of parent-child communication about sexuality and hiv/aids in sub-Saharan Africa," Reproductive Health 8(1): 25. doi: 10.1186/1742-4755-8-25.

243. African Youth Alliance. 2005. "Bringing Youth and Adults Together to Improve Adolescent Sexual and Reproductive Health in Uganda." Kampala: PATH.

244. Wekesa, Eliud, Ian Askew, and Timothy Abuya. 2018. "Ambivalence in pregnancy intentions: the effect of quality of care and context among a cohort of women attending family planning clinics in Kenya," PLOS ONE 13(1): e0190473. doi: 10.1371/journal.pone.0190473.

245. Assaf, Shireen, Wenjuan Wang, and Lindsay Mallick. 2017. "Quality of care in family planning services in Senegal and their outcomes," BMC Health Services Research 17(1). doi: 10.1186/s12913-017-2287-z.

246. Kim, Young Mi, et al. 2005. "Promoting informed choice: evaluating a decision-making tool for family planning clients and providers in Mexico," International Family Planning Perspectives 31(4): 162-71. doi: 10.1363/ifpp.31.162.05.

247. Kim, Young Mi, Claudia Davila, Carolina Tellez, and Adrienne Kols. 2007. "Evaluation of the World Health Organization's Family Planning Decision-Making Tool: improving health communication in Nicaragua," Patient Education and Counseling 66(2): 235-42. doi: 10.1016/j.pec.2006.12.007.

248. Bruce, Linda, and Antonieta Martin. 2008. Balanced Counseling Strategy: A Toolkit for Family Planning Service Providers-Trainer's Guide. Washington, DC: Population Council.

249. Okour, Abdulhakeem M., Rami A. Saadeh, and Mona Zaqoul. 2017. "Evaluation of family planning counselling in north Jordan," Sultan Qaboos University Medical Journal 17(4): e436-43. doi: 10.18295/ squmj.2017.17.04.010. 
250. Kamhawi, Sarah, Carol Underwood, Huda Murad, and Bushra Jabre. 2013. "Client-centered counseling improves client satisfaction with family planning visits: evidence from Irbid, Jordan," Global Health, Science and Practice 1(2): 180-92. doi: 10.9745/ GHSP-D-12-00051.

251. Cooper, Chelsea M. et al. 2014. "Findings from the use of a narrative story and leaflet to influence shifts along the behavior change continuum toward postpartum contraceptive uptake in Sylhet District, Bangladesh," Patient Education and Counseling 97(3): 376-82. doi: 10.1016/j.pec.2014.09.007.

252. Ahmed, Saifuddin et al. 2015. "The effect of integrating family planning with a maternal and newborn health program on postpartum contraceptive use and optimal birth spacing in rural Bangladesh," Studies in Family Planning 46(3): 297-312. doi: 10.1111/j.1728-4465.2015.00031.x.

253. Ahmed, Salahuddin et al. 2013. “Operations research to add postpartum family planning to maternal and neonatal health to improve birth spacing in Sylhet District, Bangladesh," Global Health: Science and Practice 1(2): 262-76. doi: 10.9745/GHSP-D-13-00002.

254. Ashraf, Nava, Erica Field, Alessandra Voena, and Roberta Ziparo. 2017. "Maternal mortality risk and the gender gap in desired fertility in Zambia." Lusaka: Innovations for Poverty Action-Zambia. https:// www.poverty-action.org/sites/default/files/publications/Maternal\%20Mortality\%20Brief_2017.07.20. pdf.

255. Vouking, Marius Zambou, Christine Danielle Evina, and Carine Nouboudem Tadenfok. 2014. "Male involvement in family planning decision making in sub-Saharan Africa-what the evidence suggests," The Pan African Medical Journal 19. doi: 10.11604/ pamj.2014.19.349.5090.

256. Institute for Reproductive Health, Georgetown University. 2017. "Couples counseling in reproductive health: a review of the literature," FACT Project. Washington, D.C.: Institute for Reproductive Health, Georgetown University.

257. El-Khoury, Marianne et al. 2016. "Counseling women and couples on family planning: a randomized study in Jordan." Studies in Family Planning 47(3): 222-38. doi: 10.1111/sifp.69.

258. O’Reilly, Kevin R., Caitlin E. Kennedy, Virginia A. Fonner, and Michael D. Sweat. 2013. "Family planning counseling for women living with HIV: a systematic review of the evidence of effectiveness on contraceptive uptake and pregnancy incidence, 1990 to 2011," BMC Public Health 13: 935. doi: 10.1186/1471-2458-13-935.

259. Saeed, Gulshan Ara, Shazia Fakhar, Faisal Rahim, and Sabir Tabassum. 2008. "Change in trend of contraceptive uptake: effect of educational leaflets and counseling," Contraception 77(5): 377-81. doi: 10.1016/j.contraception.2008.01.011.

260. Tessema, Gizachew Assefa, Judith Streak Gomersall, Mohammad Afzal Mahmood, and Caroline O. Laurence. 2016. "Factors determining quality of care in family planning services in Africa: a systematic review of mixed evidence," PLOS ONE 11(11): e0165627. doi: 10.1371/journal.pone.0165627.

261. Mugisha, John Frank, and Heidi Reynolds. 2008. "Provider perspectives on barriers to family planning quality in Uganda: a qualitative study," The Journal of Family Planning and Reproductive Health Care 34(1): 37-41. doi: 10.1783/147118908783332230.

262. Braun, Rebecca et al. 2016. "An evaluation of a family planning mobile job aid for community health workers in Tanzania," Contraception 94(1): 27-33. doi: 10.1016/j.contraception.2016.03.016.

263. Huntington, Dale et al. 2010. "Impact of a service provider incentive payment scheme on quality of reproductive and child-health services in Egypt," Journal of Health, Population, and Nutrition 28(3): 273-80. doi: 10.3329/jhpn.v28i3.5556

264. Kim, Young Mi, Fitri Putjuk, Endang Basuki, and Adrienne Kols. 2000. "Self-assessment and peer review: improving Indonesian service providers' communication with clients," International Family Planning Perspectives 26(1): 4. doi: 10.2307/2648283.

265. Agha, Sohail, Aslam Fareed, and Joseph Keating. 2011. "Clinical training alone is not sufficient for reducing barriers to IUD provision among private providers in Pakistan," Reproductive Health 8(1). doi: 10.1186/1742-4755-8-40.

266. Sheeran, P., and S. Orbell. 2998. "Do intentions predict condom use? meta-analysis and examination of six moderator variables," The British Journal of Social Psychology 37(Pt 2): 231-50.

267. Free, Caroline et al. 2011. "A systematic review of randomised controlled trials of interventions promoting effective condom use," Journal of Epidemiology and Community Health 65(2): 100-110. doi: 10.1136/ jech.2008.085456 
268. Fonner, Virginia A., Caitlin E. Kennedy, Kevin R. O'Reilly, and Michael D. Sweat. 2014. "Systematic assessment of condom use measurement in evaluation of HIV prevention interventions: need for standardization of measures," AIDS and Behavior 18(12): 2374-86. doi: 10.1007/s10461-013-0655-1.

269. Wechsberg, Wendee M. et al. 2010. "Preliminary findings of an adapted evidence-based woman-focused HIV intervention on condom use and negotiation among at-risk women in Pretoria, South Africa," Journal of Prevention \& Intervention in the Community 38(2): 132-46. doi: 10.1080/10852351003640799.

270. Lopez, Laureen M. et al. 2013. "Behavioral interventions for improving condom use for dual protection," Cochrane Database of Systematic Reviews 10: CD010662. doi: 10.1002/14651858.CD010662.pub2.

271. Steiner, Markus J. et al. 2007. "Decreased condom breakage and slippage rates after counseling men at a sexually transmitted infection clinic in Jamaica," Contraception 75(4): 289-93. doi: 10.1016/j. contraception.2006.12.014.

272. PATH. 2003. Counseling on Dual Protection: STD/HIV and Pregnancy. A Guide for Project Staff, Community Health Outreach Workers (CHOWs) and Peer Educators (PEs). PATH.

273. Schwandt, Hilary M, Joanna Skinner, Adel Takruri, and Douglas Storey. 2015. "The integrated gateway model: a catalytic approach to behavior change," International Journal of Gynecology \& Obstetrics 130(Suppl 3): E62-68.

274. The Health Communication Capacity Collaborative. 2016. "Factors impacting the effectiveness of health care worker behavior change a literature review." Baltimore: Johns Hopkins Center for Communication Programs. http://healthcommcapacity.org/ wp-content/uploads/2016/05/HCW-Barriers-andFacilitators-Lit-Review_May2016.pdf.

275. Mullainathan, Sendhil, and Eldar Shafir. 2013. Scarcity: Why Having Too Little Means so Much. New York: Times Books.

276. Lavie, Nilli. 2005. "Distracted and confused?: selective attention under load," Trends in Cognitive Sciences 9(2): 75-82.

277. Simons, Daniel J., and Christopher F. Chabris. 1999. "Gorillas in our midst: sustained inattentional blindness for dynamic events," Perception 28(9): 1059-74.
278. Drew, Trafton, Melissa L.-H. Võ, and Jeremy M. Wolfe. 2013. "The invisible gorilla strikes again: sustained inattentional blindness in expert observers," Psychological Science 24(9): 1848-53.

279. Nisbett, Richard E., and Lee Ross. 1980. Human Inference: Strategies and Shortcomings of Social Judgment. Prentice Hall.

280. Weinstein, Neil D. 1980. "Unrealistic optimism about future life events," Journal of Personality and Social Psychology 39(5): 806.

281. Karlsson, Niklas, George Loewenstein, and Duane Seppi. 2009. "The ostrich effect: selective attention to information," Journal of Risk and Uncertainty 38(2): 95-115. doi: 10.1007/s11166-009-9060-6.

282. Samuelson, William, and Richard Zeckhauser. 1988. "Status quo bias in decision making," Journal of Risk and Uncertainty 1(1): 7-59.

283. Brewer, William F., and Glenn V. Nakamura. 1984. "The nature and functions of schemas," Center for the Study of Reading Technical Report 325. http://www. ideals.illinois.edu/bitstream/handle/2142/17542/ ctrstreadtechrepv01984i00325_opt.pdf?s.

284. Nisbett, Richard E., and Timothy D. Wilson. 1977. "The Halo Effect: evidence for unconscious alteration of judgments," Journal of Personality and Social Psychology 35(4): 250.

285. Chandon, Pierre, and Brian Wansink. 2007. "The biasing health halos of fast-food restaurant health claims: lower calorie estimates and higher side-dish consumption intentions," Journal of Consumer Research 34(3): 301-14. doi: 10.1086/519499.

286. Banteyerga, Hailom et al. 2004. "Exploring HIV and AIDS stigma and related discrimination in Ethiopia: causes, manifestations, consequences, and coping mechanisms." Addis Ababa: Miz-Hasab Research Center, 6-69.

287. Rowe, Alexander K, Don de Savigny, Claudio F Lanata, and Cesar G Victora. 2005. "How can we achieve and maintain high-quality performance of health workers in low-resource settings?," The Lancet 366(9490): 1026-35. doi: 10.1016/S0140-6736(05)67028-6.

288. Bluestone, Julia et al. 2013. "Effective in-service training design and delivery: evidence from an integrative literature review," Human Resources for Health 11(1). doi: 10.1186/1478-4491-11-51. 
289. Witter, Sophie, Atle Fretheim, Flora L Kessy, and Anne Karin Lindahl. 2012. "Paying for performance to improve the delivery of health interventions in low- and middle-income countries," Cochrane Database of Systematic Reviews (2): CD007899. doi: 10.1002/14651858.CD007899.pub2.

290. Central Statistical Office, Zambia, Ministry of Health, and ICF International. 2015. "Zambia Demographic and Health Survey 2013-14." Rockville, Maryland, USA: Central Statistical Office, Ministry of Health, and ICF International. https://dhsprogram.com/publications/publication-fr304-dhs-final-reports.cfm.

291. World Health Organization, UNICEF, United Nations, Department of Economic and Social Affairs, Population Division, and World Bank. 2015. Trends in Maternal Mortality: 1990 to 2015 : Estimates by WHO, UNICEF, UNFPA, World Bank Group and the United Nations Population Division. Geneva: WHO. http://www.who.int/reproductivehealth/ publications/monitoring/maternal-mortality-2015/ en/.

292. United Nations Inter-agency Group for Child Mortality, and Estimation (UN IGME). 2017. "Levels \& Trends in Child Mortality: Report 2017, Estimates Developed by the UN Inter-Agency Group for Child Mortality Estimation." New York: United Nations Children's Fund. https://www.unicef.org/publications/files/ Child_Mortality_Report_2017.pdf.

293. Serbanescu, Florina et al. 2017. "Rapid reduction of maternal mortality in Uganda and Zambia through the Saving Mothers, Giving Life Initiative: results of year 1 evaluation," BMC Pregnancy and Childbirth 17(42). doi: 10.1186/s12884-017-1222-y.

294. Owens, Lauren et al. 2015. "The state of routine and emergency obstetric and neonatal care in Southern Province, Zambia," International Journal of Gynecology \& Obstetrics 128(1): 53-57. doi: 10.1016/j. ijgo.2014.07.028.

295. "Assessing the impact of the safe childbirth checklist and systems coaching on health worker practices in Zambia," n.d.

296. Sialubanje, Cephas, Karlijn Massar, Davidson H. Hamer, and Robert AC Ruiter. 2015. "Reasons for home delivery and use of traditional birth attendants in rural Zambia: a qualitative study," BMC Pregnancy and Childbirth 15(1). doi: 10.1186/s12884-015-0652-7.

297. Topp, Stephanie M, and Julien M Chipukuma. "A qualitative study of the role of workplace and interpersonal trust in shaping service quality and responsiveness in Zambian primary health centres," Health Policy and Planning 31(2): 192-204. doi: 10.1093/heapol/czv041.

298. Sacks, Emma, and Mary V. Kinney. 2015. "Respectful maternal and newborn care: building a common agenda," Reproductive Health 12(1). doi: 10.1186/ s12978-015-0042-7. 


\section{Population Council}

4301 Connecticut Ave., NW | Suite 280

Washington, DC 20008

+12022379400

breakthroughactionandresearch.org 